\title{
Bloch-Wave Homogenization for a Spectral Problem in Fluid-Solid Structures
}

\author{
Grégoire Allaire \& Carlos Conca
}

\author{
Communicated by R. V. KoHN
}

\begin{abstract}
This paper is concerned with the study of the vibrations of a coupled fiuid-solid periodic structure. As the period goes to zero, an asymptotic analysis of the spectrum (i.e., the set of eigenfrequencies) is performed with the help of a new method, the so-called Bloch-wave homogenization method (which is a blend of two-scale convergence and Bloch-wave decomposition). The limit spectrum is made of three parts: the macroscopic or homogenized spectrum, the microscopic or Bloch spectrum, and the boundary-layer spectrum. The two first parts are completely characterized: The homogenized and the Bloch spectra are purely essential, and have a band structure. The boundary-layer spectrum is shown to be empty in the special case of periodic boundary condition.
\end{abstract}

\section{Contents}

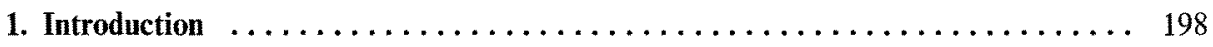

1.1. Presentation of the main results $\ldots \ldots \ldots \ldots \ldots \ldots \ldots \ldots \ldots \ldots$

1.2. Physical background and mathematical setting of the problem . . . . . 202

2. Classical homogenization: a macroscopic limit operator $\ldots \ldots \ldots \ldots \ldots$

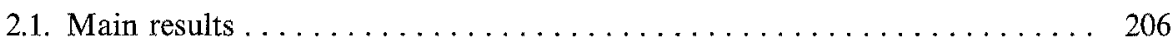

2.2. Convergence of the classical homogenization process . . . . . . . 212

2.3. Spectrum of the limit operator . . . . . . . . . . . . . . 219

3. Bloch-wave homogenization: a coupled macro-microscopic limit operator . . . . 223

3.1. Motivation and discrete Bloch waves $\ldots \ldots \ldots \ldots \ldots \ldots \ldots \ldots \ldots . \ldots \ldots$

3.2. Main results ............................... 227

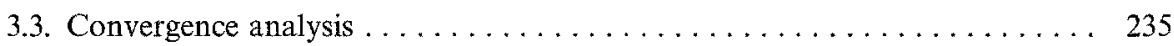

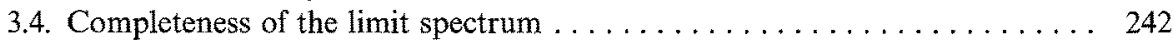




\section{Introduction}

\subsection{Presentation of the main results}

This paper is devoted to the asymptotic analysis of the spectrum of an elliptic operator defined in a periodic bounded domain whose period goes to zero. The motivation of such a problem is the study of the vibration frequencies of a coupled system of solid tubes immersed in a perfect incompressible fluid. A detailed description of this problem is given in the second part of this introduction. For the moment, we content ourselves with giving a brief statement of the problem, and we focus instead on the main tools and results obtained hereafter.

We consider a periodic bounded domain $\Omega_{\varepsilon}$ obtained from a fixed domain $\Omega$ by removing a collection of identical, periodically distributed holes $\left(T_{p}^{\varepsilon}\right)_{1 \leqq p \leqq n(\varepsilon)}$. The distance between adjacent holes, as well as their size, are both of the order of $\varepsilon$. Correspondingly, the number of holes $n(\varepsilon)$ is of the order of $\varepsilon^{-N}$, where $N$ is the spatial dimension. The spectral problem we are interested in is to find the real eigenvalues $\lambda_{\varepsilon}$ and the corresponding normalized eigenvectors $u_{\varepsilon}$ that satisfy

$$
\begin{aligned}
-\Delta u_{\varepsilon} & =0 & & \text { in } \Omega_{\varepsilon}, \\
\lambda_{\varepsilon} \frac{\partial u_{\varepsilon}}{\partial n} & =\varepsilon^{-N} \boldsymbol{n} \cdot \int_{\partial T_{p}^{\varepsilon}} u_{\varepsilon} n d s & & \text { on } \partial T_{p}^{\varepsilon} \text { for } 1 \leqq p \leqq n(\varepsilon), \\
u_{\varepsilon} & =0 & & \text { on } \partial \Omega,
\end{aligned}
$$

where $\boldsymbol{n}$ denotes the exterior unit normal to $\Omega_{\varepsilon}$. This model is due to J. PLANChard $[27,28]$, and it has already attracted the attention of several authors (see $[1,11,12])$. We call $\sigma_{\varepsilon}$ the subset of $\mathbb{R}$ made of such eigenvalues $\lambda_{\varepsilon}$. A key feature of this model is that $\sigma_{\varepsilon}$ is made of a finite number (proportional to the number $n(\varepsilon)$ of holes) of eigenvalues which are uniformly bounded away from zero and from infinity (see Proposition 1.2.1 and Lemma 1.2.2 below). As the period $\varepsilon$ goes to zero, this spectrum $\sigma_{\varepsilon}$ converges to a limit set $\sigma_{\infty} \subset \mathbb{R}$. The asymptotic analysis of this spectral problem is to find a characterization of the limit spectrum $\sigma_{\infty}$.

To our knowledge, there are two methods to study the asymptotic behavior of the partial differential equation (1). As it is presented, the most obvious one is the homogenization method for periodic structures (see, e.g., $[5,6,33]$ ). However, it turns out that, in the present situation, it is also the most difficult to work out, and so far, no significant progress has been made in this direction. Consequently, the previous works of $\mathrm{C}$. CONCA, M. VANNINATHAN, and their co-workers (see $[1,10,12]$ ), have used a second method, the so-called Bloch-wave method, also called the non-standard homogenization procedure in [11] (for other applications of this method see, e.g., [31]). The differences in the application of these two methods are easy to understand. In the homogenization process the overall domain $\Omega$ is kept fixed while the size $\varepsilon$ of the microstructure goes to zero (see Figure 1 ). Thus, in the limit, the homogenized fluid domain is $\Omega$ where the tubes have disappeared, but their influence is still manifest in the effective (or homogenized) coefficients of the limit equation. On the contrary, for the Bloch-wave method, the 

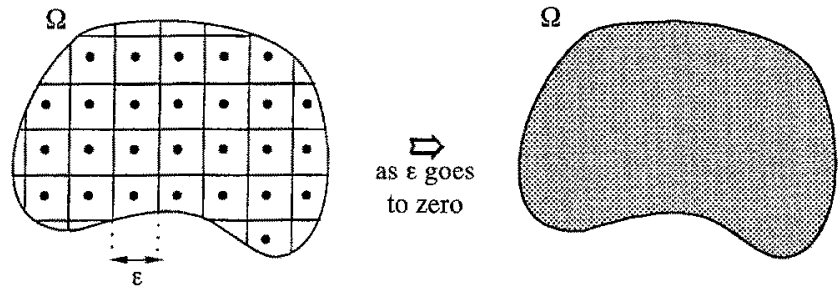

Fig. 1. Homogenization process
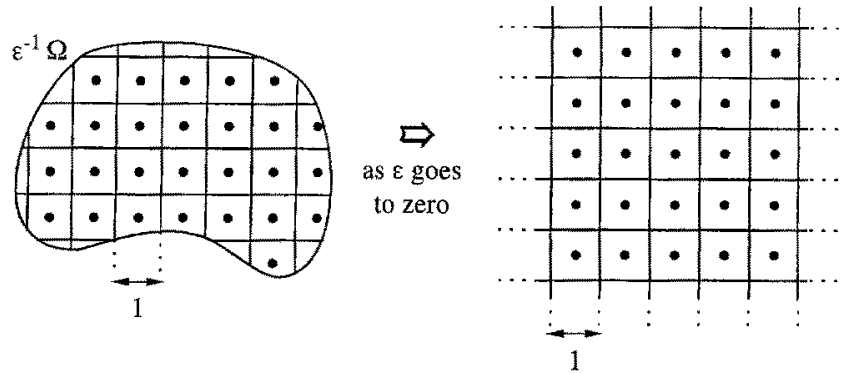

Fig. 2. Bloch-wave limit

$\varepsilon$-network of tubes is first rescaled to size 1 . Thus, as $\varepsilon$ goes to zero, the tubes remain fixed with a constant unit size, while the boundary of the fluid domain $\varepsilon^{-1} \Omega_{\varepsilon}$ goes to infinity. In the limit, the fluid domain is the entire space $\mathbb{R}^{N}$ minus an infinite periodic arrangement of unit tubes (see Figure 2). Then, the limit problem is amenable to the celebrated Bloch-wave decomposition (also known as the Floquet decomposition; see the original work of F. BLOCH [7], or the first mathematical proofs in $[14,24,39]$, or the books $[6,29]$.

The first goal of this paper is to homogenize the spectral problem (1). This task is achieved in Section 2 by using the two-scale convergence method introduced by G. Allaire [2] and G. Nguetseng [23]. More precisely, we introduce a compact self-adjoint operator $\tilde{S}_{\varepsilon}$ acting on $L^{2}(\Omega)^{N}$, having characteristic values which coincide with the eigenvalues of (1). We prove that this operator $\tilde{S}_{\varepsilon}$ converges strongly but not uniformly to a non-compact self-adjoint operator $S$ in $L^{2}(\Omega)^{N}$ (see Theorem 2.1.1). We characterize the spectrum $\sigma(S)$ of the homogenized operator $S$ (see Theorems 2.1 .4 and 2.1.5), but the "poor" convergence of the sequence $\tilde{S}_{\varepsilon}$ implies merely that

$$
\sigma(S) \subset \sigma_{\infty}
$$

where the inclusion is generically strict. As a matter of fact, the spectrum $\sigma(S)$ of the homogenized problem does not coincide with the limit spectrum obtained by the Bloch-wave method in [12]. Therefore, the second goal of this paper is to understand this discrepancy between the "homogenized" and "Bloch" spectra, and to recover all the limit set $\sigma_{\infty}$. 
This goal is achieved in Section 3 by introducing a new method, called the Bloch-wave homogenization method, which is a blend of the two-scale convergence and of the Bloch-wave decomposition. This allows us to improve the analysis of Section 2 in the sense that we are able to recover simultaneously the homogenized and the Bloch spectra. Since we believe this new method is of intrinsic interest, we briefly give a flavor of its main underlying idea. The starting point is the definition of the "usual" two-scale convergence where the period of oscillations of the test functions is a multiple $K$ of the unit cube $Y=[0,1]^{N}$.

Theorem. Let $u_{\varepsilon}$ be a bounded sequence in $L^{2}(\Omega)$. Let $K$ be any positive integer. There exist a subsequence (still denoted by $\varepsilon$ ) and a two-scale limit $u_{0}^{K}(x, y) \in L^{2}(\Omega \times K Y)$ such that $u_{\varepsilon}(x)$ two-scale converges to $u_{0}^{K}(x, y)$ in the sense that

$$
\lim _{\varepsilon \rightarrow 0} \int_{\Omega} u_{\varepsilon}(x) \phi\left(x, \frac{x}{\varepsilon}\right) d x=\frac{1}{|K Y|} \int_{\Omega} \int_{K Y} u_{0}^{K}(x, y) \phi(x, y) d x d y
$$

for any function $\phi(x, y)$ in $\mathscr{D}\left[\Omega ; C_{\#}^{\infty}(K Y)\right]$.

Note that in the convergence (2) the test function $\phi$ is $K Y$-periodic in the variable $y$. Since the choice of the integer $K$ is arbitrary, it remains to find a relation between all the different two-scale limits $u_{0}^{K}$. This is done by introducing the so-called discrete Bloch-wave decomposition (see [1]).

Theorem. Let $u_{\varepsilon}$ be a sequence in $L^{2}(\Omega)$ which, for any integer $K$, two-scale converges to a limit $u_{0}^{K}(x, y) \in L^{2}(\Omega \times K Y)$. There exists a countable family of complex-valued functions of $L^{2}\left(\Omega ; L_{\#}^{2}(Y)\right)$, denoted by $\left(u_{j / K}(x, y)\right)_{0 \leqq j \leqq K-1<+\infty}$ (where $j$ is a multi-index, the components of which vary between 0 and $K-1$, while $K$ run in $\mathbb{N}$ ), such that, for any $K$,

$$
u_{0}^{K}(x, y)=\sum_{0 \leqq j \leqq K-1} u_{j / K}(x, y) e^{2 \pi i \theta_{j} \cdot y}, \quad \text { where } \theta_{j}=\frac{j}{K} .
$$

Furthermore, the Parseval identity holds:

$$
\frac{1}{|K Y|} \int_{\Omega} \int_{K Y}\left|u_{0}^{K}(x, y)\right|^{2} d x d y=\sum_{0 \leqq j \leqq K-1} \int_{Y}\left|u_{j / K}(x, y)\right|^{2} d x d y .
$$

Remark that, as $j$ and $K$ vary over their range, the so-called Bloch frequencies $j / K$ become dense in $[0,1]^{N}$, and the above discrete Bloch-wave decomposition becomes very "close" to the usual continuous one. Of course, two Bloch components $u_{j / K}$ and $u_{j^{\prime} / K^{\prime}}$ coincide if their corresponding frequencies $j / K$ and $j^{\prime} / K^{\prime}$ are equal. Therefore, the Bloch components $u_{j / K}(x, y)$ actually depend on three variables: the macroscopic variable $x \in \Omega$, the microscopic $y \in[0,1]^{N}$, and the Bloch variable $\theta_{j}=j / K \in[0,1]^{N}$. It is easily seen that this approach combines the advantages of both the homogenization and the Bloch-wave method. 
More precisely in Section 3, we introduce a compact self-adjoint operator $S_{\varepsilon}^{K}$ acting on $L^{2}(\Omega \times K Y)^{N}$, having characteristic values which coincide with the eigenvalues of the spectral problem (1). We prove that it converges strongly to a non-compact self-adjoint operator $S^{K}$ in $L^{2}(\Omega \times K Y)^{N}$ (see Theorem 3.2.1). As $K$ goes to infinity, the spectrum of the limit operator $S^{K}$ converges to the union of the previous homogenized spectrum $\sigma(S)$ and of the Bloch spectrum $\sigma_{\text {Bloch }}$ (see (70)). However, the convergence of the sequence $S_{\varepsilon}^{K}$ is still not uniform and we merely conclude that

$$
\lim _{K \rightarrow+\infty} \sigma\left(S_{K}\right)=\left(\sigma(S) \cup \sigma_{\mathrm{Bloch}}\right) \subset \sigma_{\infty}
$$

Having reconciled the Bloch-wave and the homogenization methods, we may hope that it is enough to completely characterize the limit spectrum $\sigma_{\infty}$ of the spectral problem (1). It turns out that it is not exactly the case because there may exist some sequences of eigenvectors of (1) which concentrate near the boundary $\partial \Omega$ of the domain. These sequences, which behave like boundary layers, are captured neither by the homogenization method (since they converge to zero inside the domain), nor by the Bloch-wave method (since it does not take into account a possible interaction between the boundary and the periodic network of tubes). However, for any other type of sequences of eigenvectors (not concentrating on the boundary), the limits of the corresponding sequences of eigenvalues actually belong to the spectrum of the homogenized problem or to the Bloch spectrum. In other words, the main theorem of this paper is the following completeness result (see Theorem 3.2.9).

Theorem. Denote by $\sigma_{\text {boundary }}$ the set of all limits of sequences of eigenvalues such that the corresponding sequences of eigenvectors concentrate on the boundary $\partial \Omega$. The limit spectrum $\sigma_{\infty}$ is precisely given by

$$
\sigma_{\infty}=\sigma(S) \cup \sigma_{\text {Bloch }} \cup \sigma_{\text {boundary }} .
$$

In general, we are unable to characterize $\sigma_{\text {boundary. }}$ We suspect its definition depends on the sequence of periods $\varepsilon$ (a fact which is reminiscent of a recent work of F. SANTOSA \& M. Vogelius [34]). However, when the domain is exactly made of an integral number of periodicity cells, we are able to adapt our Bloch-wave homogenization method to take care of the boundary layers. The analysis of $\sigma_{\text {boundary }}$ in this case will be the focus of a future paper [3]. For the moment, we make the obvious remark that, if the domain $\Omega$ is a torus (i.e., a compact manifold without boundary), then there is no contribution of boundary layers in the limit spectrum.

Corollary. Let $\Omega$ be a parallelpiped, $] 0, L_{1}[\times] 0, L_{2}[\times \cdots] 0, L_{N}[$, where the $\left(L_{p}\right)_{1 \leqq p \leqq N}$ are positive integers. Define the sequence of periods $\varepsilon_{n}=1 / n$. Assume that the unit tube in the periodic cell has cubic symmetry, or replace the Dirichlet boundary condition in the spectral problem (1) by a periodicity condition. Then, the limit spectrum reduces to

$$
\sigma_{\infty}=\sigma(S) \cup \sigma_{\mathrm{Bloch}}
$$


The proof of this completeness theorem is the focus of Section 3.4. It involves a new technical tool: the so-called Bloch and rescaled Bloch measures. Their purpose is to quantify the amount of oscillations for any sequence of eigenvectors of the spectral problem. They act as a filter on the length scale and the directions of propagation of the oscillations. The Bloch measure selects only the oscillations on the $\varepsilon$ scale and distinguishes their corresponding Bloch frequencies. Although specific to the present context, it can be seen as a type of Wigner, or semi-classical, measure (see [16] and [20]). The rescaled Bloch measure is sensitive only to those oscillations which have a length scale larger than $\varepsilon$, and sorts them out according to their directions (in Fourier space). It is again specific to the present situation, but it obviously bears some resemblance to the $H$-measures of P. GÉRARD [15] and L. TARTAR [37].

Our analysis of the limit spectrum $\sigma_{\infty}$ leaves open three important questions. The first is to characterize the "boundary layer" spectrum $\sigma_{\text {boundary }}$. As already said, this is the focus of our next paper [3] in the case of a domain built with entire periodic cells. However, for a general domain with a smooth boundary (not coinciding with the cell boundaries), we are helpless in the matter. The second question is to find the rate of convergence of $\sigma_{\varepsilon}$ to $\sigma_{\infty}$. The answer is unclear, and as before we suspect it depends on the form of the boundary $\partial \Omega$ and on the sequence of periods $\varepsilon$. Such effects occur in the case of the standard wave equation in a periodic domain, as recognized by F. SANTOSA \& M. Vogelius [34]. The third open question is to understand the consequences of our spectral analysis in terms of the associated time-dependent problem (a wave-type equation; see (6) below). This is the topic of future research, and we hope it could shed some light on questions of geometrical optics. Let us also indicate that we have recently applied our Bloch-wave homogenization method to study the asymptotic behavior of the spectrum of the wave equation in a periodic domain [4].

We conclude this subsection by warning the reader that the first part of this introduction has deliberately been kept to a minimal size. Nevertheless, a detailed presentation and discussion of our results is available by simply reading in a first pass the Subsections 1.2, 2.1,3.1, and 3.2 (the remaining subsections are devoted to the proofs). Specifically, Subsection 1.2 furnishes a complete description of the spectral problem (1). Subsection 2.1 gives the main results of the classical homogenization process for (1). Then, Subsection 3.1 introduces the Bloch waves, and Subsection 3.2 contains the final results of our Bloch-wave homogenization method, along with many additional comments (see Remarks 3.2.10 to 3.2.15).

\subsection{Physical background and mathematical setting of the problem}

We begin with the definition of the geometry of the fluid domain. As usual in periodic homogenization, we first define a unit cell, which, upon rescaling to size $\varepsilon$ (a small positive parameter), becomes the period of a periodic domain. Let $Y=(0 ; 1)^{N}$ be the unit cube and $T$ be a smooth, simply connected, closed set, with a non-empty interior, strictly included in $Y$ (i.e., such that $T$ does not touch the 
boundaries of the cell $Y$ ). We call the set $Y^{*}=Y \backslash T$ the fluid cell, and the set $T$ the reference hole (or rod).

Introducing a smooth, bounded, open set $\Omega$ in $\mathbb{R}^{N}$ and a sequence of positive parameters $\varepsilon$ going to zero, for each value of $\varepsilon$ we define a fluid domain $\Omega_{\varepsilon}$ obtained by removing from the reference domain $\Omega$ a collection of perforations $\varepsilon T$ distributed in a periodic manner with period $\varepsilon Y$. More precisely, we denote by $\left(T_{p}^{g}\right)$ the family of all translates of the hole $\varepsilon T$ by vectors $\varepsilon p$ (where $p$ is a multi-index in $\mathbb{Z}^{N}$ ), and by $\left(Y_{p}^{\varepsilon}\right)$ the corresponding family of cells. Analogously, $\left(\Gamma_{p}^{\varepsilon}\right)$ denotes the corresponding family of boundaries $\varepsilon \partial T$. We consider only those cells which are strictly included in the domain $\Omega$ so that no hole meets the boundary $\partial \Omega$, and even more importantly, so that each hole lies at a distance larger than the order of $\varepsilon$ from $\partial \Omega$. It is easily seen that the total number of holes $n(\varepsilon)$ is asymptotically equal to $\varepsilon^{-N}|\Omega|$. Then, the fluid domain is defined by

$$
\Omega_{\varepsilon}=\Omega_{\varepsilon} \backslash \bigcup_{p=1}^{n(\varepsilon)} T_{p}^{\varepsilon}
$$

As we shall soon explain, the underlying physical problem of fluid-solid interactions is purely two-dimensional. Nevertheless, since from a mathematical point of view there is no conceptual difficulty in higher dimensions, we shall state all our results in any space dimension $N \geqq 2$. We consider the simplest model of vibrations of a tube-bundle immersed in a perfect incompressible fluid inside a cavity with a constant planar section $\Omega$ (this model is mainly due to J. Planchard [27, 28]; more complex models are available in, e.g., [11]). A tube bundle is made of parallel tubes, long enough for three-dimensional effects to be ignored, which can move transversally under the action of the fluid pressure and of repelling forces created by some binding device. The holes $T_{p}^{\varepsilon}$ represent the cross sections of the tubes and $\Omega_{\varepsilon}$ is the part of $\Omega$ occupied by the fluid.

When such a vibrating tube bundle is immersed in the fluid, both the tubes and the fluid vibrate. The result is a non-stationary partial differential equation in the fluid region coupled with a system of ordinary differential equations which represents the oscillations of the tubes. The fluid-solid interactions are taken into account by the coupling between the partial differential equation and the system of ordinary differential equations.

More precisely, in the model we study in this paper the tubes are also assumed to be rigid and only small oscillations of the fluid around the state of rest are allowed. The velocity $U_{\varepsilon 0}$ of the fluid derives then from a potential function $u_{\varepsilon 0}=u_{\varepsilon 0}(x, t)$. Since the fluid is incompressible, its motion is governed by the Laplace equation

$$
\Delta u_{\varepsilon 0}=0 \quad \text { in } \Omega_{\varepsilon} \times \mathbb{R} .
$$

The fluid is not allowed to escape the cavity and so $u_{\varepsilon 0}$ satisfies

$$
\frac{\partial u_{\varepsilon 0}}{\partial n}=0 \quad \text { on } \partial \Omega \times \mathbb{R} .
$$


On each $\Gamma_{p}^{\varepsilon}$, the normal velocity of the fluid should coincide with the normal component of the tube's velocity. Thus we have

$$
\frac{\partial u_{\varepsilon 0}}{\partial n}=\frac{d r_{0 p}}{d t} \cdot n \quad \text { on } \Gamma_{p}^{\varepsilon} \times \mathbb{R}, \quad p=1, \ldots, n(\varepsilon),
$$

where $n$ is the outward unit normal on the boundary of $\Omega_{\varepsilon}$ and $r_{0 p}(t)$ is the transverse displacement vector at the instant $t$ of the $p$-th tube which, due to the assumption of rigidity, depends only on $t$. It is furthermore assumed that there is no interaction between the tubes and that the motion of tube $p$ is that of a simple harmonic oscillator with a forcing term modeled by its interaction with the fluid. Since the fluid is assumed to be perfect, this term depends only on the pressure $p_{\varepsilon 0}$ of the fluid. More exactly, $\boldsymbol{r}_{0 p}$ satisfies the second-order ordinary differential equation

$$
m \frac{d^{2} \boldsymbol{r}_{0 p}}{d t^{2}}+k r_{0 p}=\int_{\Gamma_{p}^{\varepsilon}} p_{\varepsilon 0}(x, t) n d s \quad \text { in } \mathbb{R},
$$

where $m$ and $k$ are two positive constants corresponding to the mass per unit length and the stiffness of the tubes, and $p_{\varepsilon 0}(x, t)$ is the pressure of the fluid at the point $x \in \Omega$ and at time $t$. Next, let us consider the Euler equation in order to link $p_{\varepsilon 0}$ and $u_{\varepsilon 0}$ :

$$
\frac{\partial \boldsymbol{U}_{\varepsilon 0}}{\partial t}+\left(\boldsymbol{U}_{\varepsilon 0} \cdot \nabla\right) \boldsymbol{U}_{\varepsilon 0}+\frac{1}{\rho} \nabla p_{\varepsilon 0}=0,
$$

where $\rho>0$ is the density of the fluid. Given that only small oscillations are being considered, linearizing the Euler equation, we obtain the well-known Bernoulli relationship

$$
p_{\varepsilon 0}=-\rho \frac{\partial u_{\varepsilon 0}}{\partial t}+c(t)
$$

where $c(t)$ is an arbitrary constant (in space, not in time). As usual in vibration models, we seek sinusoidal solutions of the form

$$
u_{\varepsilon 0}(x, t)=u_{\varepsilon} e^{i \omega_{\varepsilon} t}, \quad r_{0 p}(t)=r_{p} e^{i \omega_{\varepsilon} t}
$$

where $\omega_{\varepsilon}$ is the unknown (resonant) vibration frequency of the coupled system and $i$ is the usual square root of -1 . We can solve explicitly the ordinary differential equation for $r_{0 p}$ and get

$$
r_{0 p}(t)=-\frac{i \rho \omega_{\varepsilon} e^{i \omega_{\varepsilon} t}}{k-m \omega_{\varepsilon}^{2}} \int_{\Gamma_{p}} u_{\varepsilon} n d s
$$

It is now possible to eliminate the unknowns $\boldsymbol{r}_{0 p}$. To simplify the notation, we define a rescaled frequency

$$
\lambda_{\varepsilon}=\frac{k-m \omega_{\varepsilon}^{2}}{\varepsilon^{N} \rho \omega_{\varepsilon}^{2}}
$$


To avoid the problem of having the potential $u_{\varepsilon}$ defined up to a constant, we replace the physical Neumann boundary condition (5) on $\partial \Omega$ by the more convenient Dirichlet one. As we shall see below (cf. Remark 2.1.7), this change has basically no influence on the results, and greatly simplifies the exposition. Then, $\left(\lambda_{\varepsilon}, u_{\varepsilon}\right)$ has to be a solution of the following spectral problem in $\Omega_{\varepsilon}$

$$
\begin{aligned}
-\Delta u_{\varepsilon} & =0 & & \text { in } \Omega_{\varepsilon}, \\
\lambda_{\varepsilon} \frac{\partial u_{\varepsilon}}{\partial n} & =\frac{1}{\left|Y_{p}^{\varepsilon}\right|} n \cdot \int_{\Gamma_{p}^{\varepsilon}} u_{\varepsilon} n d s & & \text { on } \Gamma_{p}^{\varepsilon} \text { for } 1 \leqq p \leqq n(\varepsilon), \\
u_{\varepsilon} & =0 & & \text { on } \partial \Omega,
\end{aligned}
$$

where $\boldsymbol{n}$ is the exterior unit normal on the boundary of $\Omega_{\varepsilon}$.

To obtain the solutions of (7), we introduce a finite-dimensional operator $S_{\varepsilon}$, mapping the space $\mathbb{R}^{N n(\varepsilon)}$ into itself, whose characteristic values coincide with the eigenvalues $\lambda_{\varepsilon}$ of (7). A vector $s$ in $\mathbb{R}^{N n(\varepsilon)}$ is decomposed into components $\left(\boldsymbol{s}_{p}\right)_{1 \leqq p \leqq n(\varepsilon)}$, each $\boldsymbol{s}_{p} \in \mathbb{R}^{N}$ being associated with the corresponding hole $T_{p}^{\varepsilon}$. This operator $S_{\varepsilon}$ is then defined by

$$
S_{\varepsilon} s=\left(\frac{1}{\left|Y_{p}^{\varepsilon}\right|} \int_{\Gamma_{p}^{\varepsilon}} u_{\varepsilon} n d s\right)_{1 \leqq p \leqq n(\varepsilon)} \quad \text { for any } s \in \mathbb{R}^{N n(\varepsilon)},
$$

where $u_{\varepsilon}$ is now the unique solution in $H^{1}\left(\Omega_{\varepsilon}\right)$ of the boundary-value problem

$$
\begin{aligned}
-\Delta u_{\varepsilon} & =0 & & \text { in } \Omega_{\varepsilon}, \\
\frac{\partial u_{\varepsilon}}{\partial n} & =s_{p} \cdot \boldsymbol{n} & & \text { on } \Gamma_{p}^{\varepsilon} \text { for } 1 \leqq p \leqq n(\varepsilon), \\
u_{\varepsilon} & =0 & & \text { on } \partial \Omega,
\end{aligned}
$$

Remark that this choice of the operator $S_{\varepsilon}$ is somewhat arbitrary, but this nonuniqueness is not important at this point.

According to J. Planchard [27], C. Conca \& M. Vanninathan [12], the operator $S_{\varepsilon}$ is self-adjoint and positive-definite, and its characteristic values coincide with the spectrum of (7). More precisely, they proved

Proposition 1.2.1. There exist $N n(\varepsilon)$ positive reals $0<\lambda_{\varepsilon}(1) \leqq \ldots \leqq \lambda_{\varepsilon}(N n(\varepsilon))$ (not necessarily distinct) and $N n(\varepsilon)$ non-zero functions $u_{\varepsilon}(1), \ldots, u_{\varepsilon}(N n(\varepsilon))$ in $H^{1}\left(\Omega_{\varepsilon}\right)$ such that

1. for each $j=1, \ldots, N n(\varepsilon),\left[\lambda_{\varepsilon}(j), u_{\varepsilon}(j)\right]$ is a solution of $(7)$,

2. the pairs $\left[\lambda_{\varepsilon}(j), u_{\varepsilon}(j)\right]_{1 \leqq j \leqq N n(\varepsilon)}$ describe all the solutions of $(7)$.

An important feature of the spectrum of $S_{\varepsilon}$ is that it is bounded away from zero and infinity, uniformly in $\varepsilon$. This behavior is deduced from the following lemma, the proof of which can be found in Section 2.2 (a similar result can also be found in [12]). 
Lemma 1.2.2. There exist two positive constants $c$ and $C$ with $0<c \leqq C<+\infty$, which do not depend on $\varepsilon$, such that

$$
c \leqq \lambda_{\varepsilon}(1) \leqq \cdots \leqq \lambda_{\varepsilon}(N n(\varepsilon)) \leqq C .
$$

The determination of the spectral eigenvalues $\lambda_{\varepsilon}$ is of great importance in many applications (see [27] and references therein). However, for small $\varepsilon$ the number of eigenvalues is very large and their precise values are irrelevant. Rather, we are interested in the qualitative asymptotic behavior of the spectrum. Thus, the goal of this paper is to study the limit of the spectrum $\left[\lambda_{\varepsilon}(j), u_{\varepsilon}(j)\right]_{1 \leqq j \leqq N n(\varepsilon)}$ of $(7)$ as the period $\varepsilon$ goes to zero. In practice we achieve this goal by studying the convergence of the sequence of operators $S_{\varepsilon}$. Since these operators are not defined on the same fixed space, but rather on a sequence of spaces $\mathbb{R}^{N n(\varepsilon)}$, depending on $\varepsilon$, we shall introduce an extension $\tilde{S}_{\varepsilon}$ of each $S_{\varepsilon}$ defined on a fixed Hilbert space. This will allow us to use the classical theory of perturbations of linear operators and the powerful tools on spectral convergence developed by F. RELLrCH (see his original work [30] or modern textbooks such as [18] or [31]).

However, the question of choosing the right extension of $S_{\varepsilon}$ (i.e., of choosing in which fixed space $\mathbb{R}^{N n(\varepsilon)}$ should be embedded) is a subtle one and is addressed in great detail in Section 3.1. At this point, let us simply warn the reader that different extensions yield various limit operators, possibly with different limit spectra. This seemingly paradoxical result is due to the fact that, although $S_{\varepsilon}$ is a nice, finite-rank operator, it never converges, in any sense, to a compact limit operator. This loss of compactness is the main feature of this problem, which makes it both difficult and interesting.

\section{Classical homogenization: a macroscopic limit operator}

\subsection{Main results}

In this section, we extend the operators $S_{\varepsilon}$ to the Hilbert space $L^{2}(\Omega)^{N}$, and we study the convergence of these extensions, denoted by $\widetilde{S}_{\varepsilon}$, in the fixed space $L^{2}(\Omega)^{N}$. In some sense, it is the most natural choice of extension, since, as $\varepsilon$ goes to zero, a vector $s_{\varepsilon} \in \mathbb{R}^{N n(\varepsilon)}$, interpreted as a function constant in each cell $Y_{p}^{\varepsilon}$, "converges" to a function $s(x)$ defined in $\Omega$. This choice also has the advantage that the convergence behavior of $\tilde{S}_{\varepsilon}$ is obtained through a periodic homogenization problem, amenable to classical techniques such as two-scale convergence.

To begin with, we introduce two continuous linear operators: $P_{\varepsilon}$ which maps $L^{2}(\Omega)^{N}$ onto $\mathbb{R}^{N n(\varepsilon)}$, and $E_{\varepsilon}$ which maps $\mathbb{R}^{N n(\varepsilon)}$ into $L^{2}(\Omega)^{N}$. More precisely, they are defined by

$$
\begin{aligned}
& P_{\varepsilon}: L^{2}(\Omega)^{N} \rightarrow \mathbb{R}^{N n(\varepsilon)}, \\
& s(x) \rightarrow\left(\frac{1}{\left|Y_{g}^{\varepsilon}\right|} \int_{Y_{p}^{z}} s(x) d x\right)_{1 \leqq p \leqq n(\varepsilon),}
\end{aligned}
$$




$$
\begin{gathered}
E_{\varepsilon}: \mathbb{R}^{N n(\varepsilon)} \rightarrow L^{2}(\Omega)^{N}, \\
\left(s_{p}\right)_{1 \leqq p \leqq n(\varepsilon)} \rightarrow s(x)=\sum_{p=1}^{n(\varepsilon)} s_{p} \chi_{Y_{p}^{\varepsilon}}(x)
\end{gathered}
$$

where $\chi_{Y_{p}^{\varepsilon}}(x)$ is the characteristic function of the set $Y_{p}^{\varepsilon}$. It is easily seen that the composition $P_{\varepsilon} E_{\varepsilon}$ is nothing but the identity in $\mathbb{R}^{N n(\varepsilon)}$, while $E_{\varepsilon} P_{\varepsilon}$ is the standard projection operator from $L^{2}(\Omega)^{N}$ onto its subspace of piecewise constant functions on each cell $Y_{p}^{\varepsilon}$.

We are now in a position to define the extension $\widetilde{S}_{\varepsilon}$ by means of the following composition rule

$$
\begin{aligned}
\tilde{S_{\varepsilon}}: L^{2}(\Omega)^{N} & \rightarrow L^{2}(\Omega)^{N} \\
s(x) & \rightarrow E_{\varepsilon} S_{\varepsilon} P_{\varepsilon} s(x) .
\end{aligned}
$$

One can easily check that $E_{\varepsilon}^{*}=\varepsilon^{N} P_{\varepsilon}$ and $P_{\varepsilon}^{*}=\varepsilon^{-N} E_{\varepsilon}$ (where the symbol $*$ denotes the adjoint operator). Since $S_{\varepsilon}$ is self-adjoint and has finite rank, its extension $\tilde{S}_{\varepsilon}$ is obviously self-adjoint and has finite rank too (thus, it is compact). Since $P_{\varepsilon}$ is surjective, the spectrum $\sigma\left(\tilde{S}_{\varepsilon}\right)$ of $\tilde{S}_{\varepsilon}$ is nothing but that of $S_{\varepsilon}$ plus the eigenvalue 0 , which has an infinite multiplicity. As a consequence, in this framework the asymptotic analysis of (7) is reduced to the study of the convergence of the sequence $\tilde{S}_{\varepsilon}$ in the space of linear continuous operators on $L^{2}(\Omega)^{N}$. Our main result is a strong (but not uniform) convergence of this sequence to a non-compact limit operator $S$.

Theorem 2.1.1. The sequence of operators $\tilde{S}_{\varepsilon}$ converges strongly to a limit $S$ on $L^{2}(\Omega)^{N}$, i.e., for any function $s \in L^{2}(\Omega)^{N}$,

$$
\tilde{S}_{\varepsilon} s \rightarrow S \boldsymbol{s} \text { in } L^{2}(\Omega)^{N} \text { strongly, }
$$

and the limit operator $S$ is defined by

$$
S \boldsymbol{s}=(A-I) \nabla u-(A-\theta I) \boldsymbol{s},
$$

where I denotes the identity matrix, $\theta=\left|Y^{*}\right|$ denotes the volume fraction of fluid in the unit cell, $u$ is the unique solution in $H_{0}^{1}(\Omega)$ of the boundary-value problem

$$
\begin{aligned}
-\operatorname{div}(A \nabla u) & =\operatorname{div}((I-A) s) & & \text { in } \Omega, \\
u & =0 & & \text { on } \partial \Omega,
\end{aligned}
$$

and the matrix $A$ is defined by

$$
A_{i j}=\int_{Y^{*}}\left(\nabla_{y} w_{i}+\boldsymbol{e}_{i}\right) \cdot\left(\nabla_{\dot{y}} w_{j}+\boldsymbol{e}_{j}\right) d y
$$

where, for $1 \leqq i \leqq N, w_{i}$ is the unique solution of the so-called cell problem

$$
\begin{array}{ll}
-\operatorname{div}_{y}\left(\nabla_{y} w_{i}+\boldsymbol{e}_{i}\right)=0 & \text { in } Y^{*}, \\
\left(\nabla_{y} w_{i}+\boldsymbol{e}_{\boldsymbol{i}}\right) \cdot \boldsymbol{n}=0 & \text { on } \partial T, \\
y \rightarrow w_{i}(y) \text { is Y-periodic. } &
\end{array}
$$


The proof of Theorem 2.1.1 is given in Section 2.2. The essential ingredient for proving the strong convergence of $\widetilde{S}_{\varepsilon}$ is a careful analysis of the homogenization of the boundary-value problem (9) for the potential $u_{\varepsilon}$. Our main tool is the two-scale convergence method recently introduced by G. Allaire [2] and G. NGUETSENG [23].

Remark 2.1.2. In view of definition (13) of the limit operator $S$, it seems likely that it is not compact. This is indeed the case by virtue of Theorem 2.1.4 below. Since the operators $\tilde{S}_{\varepsilon}$ are compact, this theorem indicates the optimality of the strong convergence, in $L^{2}(\Omega)^{N}$, of this sequence. One cannot hope to prove a sharper result, namely, the uniform convergence of $\tilde{S}_{\varepsilon}$, because this convergence would imply the compactness of the limit $S$.

In order to describe the spectrum of $S$, we recall a well-known lemma on the properties of the homogenized matrix $A$.

Lemma 2.1.3. Let $A$ be the homogenized matrix defined by (15). Then, both $A$ and $(\theta I-A)$ are symmetric and positive-definite. Furthermore, if the fluid cell $Y^{*}$ has cubic symmetry, then the homogenized matrix $A$ is proportional to the identity.

Theorem 2.1.4. The limit operator $S$, defined on $L^{2}(\Omega)^{N}$ by (13), is positive-definite, self-adjoint, and not compact. Furthermore, its spectrum $\sigma(S)$ coincides with its essential spectrum. Since the matrix

$$
B(\lambda)=A-(I-A)(A+(\lambda-\theta) I)^{-1}(I-A)
$$

is diagonizable by virtue of Lemma 2.1.3, the spectrum of $S$ is exactly the set of all values of $\lambda$ such that $B(\lambda)$ has either an infinite or a zero eigenvalue, or has simultaneously positive and negative eigenvalues. Thus, the spectrum of $S$ is a finite union of intervals of $\mathbb{R}$.

We recall that the essential spectrum of a self-adjoint operator is the subset of its spectrum whose elements are not isolated eigenvalues of finite multiplicity. Thus, $S$ cannot be compact since the essential spectrum of a compact operator can contain only the single point zero. In the case where the homogenized matrix $A$ is a multiple of the identity, we can improve Theorem 2.1.4.

Theorem 2.1.5. Assume that the fluid cell $Y^{*}$ has cubic symmetry; then, by virtue of Lemma 2.1.3, the homogenized matrix $A$ is equal to $\alpha I$, with $0<\alpha \leqq \theta$. In this isotropic case, the spectrum $\sigma(S)$ of $S$ consists of two eigenvalues, $\lambda_{1} \leqq \lambda_{2}$, of infinite multiplicity:

$$
\lambda_{1}=\theta-\alpha, \quad \lambda_{2}=\theta+\frac{1-2 \alpha}{\alpha}
$$


whose eigenspaces are respectively

$$
\begin{aligned}
& E_{1}=\left\{s \in L^{2}(\Omega)^{N} \text { such that } \operatorname{div} s=0 \text { in } \Omega\right\} \\
& E_{2}=\left\{s \in L^{2}(\Omega)^{N} \text { such that } s=\nabla q \text { with } q \in H_{0}^{1}(\Omega)\right\} .
\end{aligned}
$$

The proofs of Theorems 2.1.4, 2.1.5 are given in Section 2.3.

Remark 2.1.6. It is easily seen that the direct sum of the eigenspaces $E_{1}$ and $E_{2}$ is precisely $L^{2}(\Omega)^{N}$, as it should be. From a physical point of view, $E_{1}$ is the set of all macroscopically incompressible displacements of the tubes (corresponding to a zero fluid velocity), while $E_{2}$ contains the gradient-type displacements which yield a non-zero fluid velocity. In the anisotropic case (in which $A$ is not proportional to the identity), such an orthogonal decomposition of $L^{2}(\Omega)^{N}$, with respect to $S$, is not easily available. Furthermore, the spectrum of $S$ exhibits a band structure as can be readily checked from its definition.

Remark 2.1.7. The spectrum $\sigma(S)$ is defined by an algebraic criterion involving the eigenvalues of the matrix $B(\lambda)$, regardless of the precise type of boundary condition satisfied by the potential $u$. In particular, if we change the definition of the operator $S$ by replacing the Dirichlet boundary condition on $\partial \Omega$ by a Neumann condition in (14), this does not affect its spectrum $\sigma(S)$ (considered as a subset of the real line). The same holds true for other types of "usual" boundary conditions like mixed or Fourier conditions. Of course, a value $\lambda$ in the spectrum may or may not be an eigenvalue, depending on the precise type of boundary conditions. Moreover, the definition of the corresponding eigenspaces depend also on the boundary condition in (14). However, as far as the characterization of $\sigma(S)$ is concerned, the entire analysis performed in this paper for a Dirichlet boundary condition carries over mutatis mutandis for the more physical case of a Neumann boundary condition.

It remains to see in which sense $\sigma\left(\tilde{S_{\varepsilon}}\right)$ converges to $\sigma(S)$. If the convergence of the sequence $\tilde{S}_{\varepsilon}$ were uniform, and the limit operator $S$ compact (which is not the case by virtue of Theorem 2.1.4), then it would be a classical matter to prove pointwise convergence of the eigenvalues (see, e.g., $[25,26,38]$ ). Unfortunately, the situation here is non-standard in the sense that neither $\widetilde{S}_{\varepsilon}$ nor its resolvent converge uniformly to their limits. Therefore, following the ideas of $[31,32]$, we prove the convergence of $\sigma\left(\tilde{S}_{\varepsilon}\right)$ to $\sigma(S)$ in a much weaker sense, i.e., by means of the so-called spectral families. We first recall the classical definition of a spectral family for a self-adjoint operator on a Hilbert space (for details see, e.g., $[18,31]$ ).

Proposition 2.1.8. Let $S$ be a self-adjoint operator on a Hilbert space H. Its spectral family is the unique function $\mathscr{E}(\lambda)$, defined on $\mathbb{R}$ with values in the space of orthogonal projections on $H$, satisfying the properties

1. $S=\int_{-\infty}^{+\infty} \lambda d \mathscr{E}(\lambda)$ in the sense of Stieltjes integrals.

2. $\mathscr{E}(\lambda)$ is non-decreasing, i.e., $\mathscr{E}(\lambda) \mathscr{E}(\mu)=\mathscr{E}(\min (\lambda, \mu))$. 
3. for any $u \in H, \mathscr{E}(\lambda) u$ converges strongly to 0 in $H$ when $\lambda$ goes to $-\infty$ and to $u$ in $H$ when $\lambda$ goes to $+\infty$.

4. for any $u \in H, \lambda \rightarrow \mathscr{E}(\lambda) u$ is continuous on the right in the strong topology of $H$.

An important property of the spectral family $\mathscr{E}(\lambda)$ is that the spectrum $\sigma(S)$ coincides with the set of real values $\lambda$ such that $\mathscr{E}(\lambda)$ is not constant in a neighborhood of $\lambda$. Furthermore, the points of discontinuity of $\mathscr{E}(\lambda)$ correspond to eigenvalues (of finite or infinite multiplicity), while the points where $\mathscr{E}(\lambda)$ is continuous and not constant belong to the continuous spectrum of $S$.

It is very easy to compute the spectral family of $\tilde{S}_{\varepsilon}$, but we are able to compute that of $S$ only in the isotropic case (i.e., $A=\alpha I$ ). For $1 \leqq j \leqq N n(\varepsilon)$, with $s_{j}^{\varepsilon}(x)$ denoting the normalized eigenvector associated with the eigenvalue $\lambda_{j}^{\varepsilon}$ of $\tilde{S}_{\varepsilon}$, its spectral family $\mathscr{E}_{\hat{\varepsilon}}(\lambda)$ is simply defined by

$$
\mathscr{E}_{\varepsilon}(\lambda) s(x)=\sum_{\left\{j \mid \lambda_{j}^{\hat{j}} \leq \lambda\right\}}\left(\int_{\Omega} \boldsymbol{s}_{j}^{\varepsilon}(x) \cdot \mathbf{s}(x) d x\right) \boldsymbol{s}_{j}^{\varepsilon}(x)
$$

In the isotropic case, a well-known result states that the direct sum of the eigenspaces $E_{1}$ and $E_{2}$ is exactly $L^{2}(\Omega)^{N}$. In other words, for any $s \in L^{2}(\Omega)^{N}$, there exists a unique decomposition

$$
\boldsymbol{s}=\boldsymbol{t}+\nabla q \quad \text { with div } \boldsymbol{t}=0 \text { and } q \in H_{0}^{1}(\Omega) .
$$

Then, the spectral family $\mathscr{E}(\lambda)$ of $S$ is defined by

$$
\mathscr{E}(\lambda) s= \begin{cases}0 & \text { if } \lambda<\lambda_{1} \\ \boldsymbol{t} & \text { if } \lambda_{1} \leqq \lambda<\lambda_{2} \\ \boldsymbol{s} & \text { if } \lambda_{2} \leqq \lambda\end{cases}
$$

As a direct consequence of Rellich's theorem, which implies the convergence of spectral families from the strong convergence of a sequence of self-adjoint operators (see the original work of RELLICH [30], or more recent textbooks such as $[18,31]$ ), we deduce from Theorem 2.1.1

Theorem 2.1.9. Let $\widetilde{S}_{\varepsilon}$ and $S$ be the operators on $L^{2}(\Omega)^{N}$ defined by (12) and (13) respectively. If $\lambda$ is not an eigenvalue of $S$, then the spectral family $\mathscr{E}_{\varepsilon}(\lambda)$ of $\tilde{S}_{\varepsilon}$ converges strongly to that $\mathscr{E}(\lambda)$ of $S$ as $\varepsilon$ goes to zero, in the sense that for any $s \in L^{2}(\Omega)^{N}$

$$
\mathscr{E}_{\varepsilon}(\lambda) s \rightarrow \mathscr{E}(\lambda) s \quad \text { in } L^{2}(\Omega)^{N} \text { strongly. }
$$

Remark 2.1.10. This convergence of $\mathscr{E}_{\varepsilon}(\lambda)$ holds for all values of $\lambda$ not eigenvalues of $S$. In the isotropic case, this condition rules out only the two values $\lambda_{1}$ and $\lambda_{2}$. However, in the anisotropic case we do not know the point spectrum of $S$ (see Section 2.3 for more details). Of course, we know that the spectrum $\sigma(S)$ of $S$ coincides with its essential spectrum, but this yields no information on the decomposition of $\sigma(S)$ in its point and continuous parts. Thus, it could happen, unfortunately, that the above convergence is useless on a large part of $\sigma(S)$. 
Convergence of spectral families is a sort of weak convergence for the spectrum, not so much from a physical point of view (for a more complete discussion of this type of convergence and very interesting applications, we refer to [31]). As mentioned before, we are primarily interested in the pointwise convergence of the spectrum. From Theorem 2.1.1, we can obtain such a result which, strictly speaking, is weaker than the Rellich Theorem 2.1.9 (for its proof see Section 2.3).

Proposition 2.1.11. The strong convergence of $\tilde{S}_{\varepsilon}$ to $S$ implies that

1. For any $\lambda \in \sigma(S)$, there exists a sequence $\lambda_{\varepsilon} \in \sigma\left(\tilde{S_{\varepsilon}}\right)$ such that $\lambda_{\varepsilon} \rightarrow \lambda$.

2. There may exist non-zero sequences $\lambda_{\varepsilon} \in \sigma\left(\widetilde{S_{\varepsilon}}\right)$ such that $\lambda_{\varepsilon} \rightarrow \lambda$, where the limit $\lambda$ does not belong to $\sigma(S)$. In this case, any associated sequence of normalized eigenvectors converges weakly to zero in $L^{2}(\Omega)^{N}$.

In view of this result, it seems likely that $\sigma(S)$ does not contain all possible limits of sequences in $\sigma\left(\tilde{S}_{\varepsilon}\right)$. This means that $S$ is perhaps not the "best" limit operator available. To obtain a different limit operator, the only possibility is to change the Hilbert space in which we perform the asymptotic analysis. Indeed, we are at liberty to extend the original operator $S_{\varepsilon}$, merely defined in $\mathbb{R}^{N n(\varepsilon)}$, to a space other than $L^{2}(\Omega)^{N}$. To recover the "largest" possible limit spectrum, we have to carefully choose this new extension, following the crucial hint, given by Proposition 2.1.11, that sequences of eigenvalues which "escape" in the limit from $\sigma(S)$ are associated with eigenvectors converging weakly to zero in $L^{2}(\Omega)^{N}$. The usual belief that weak convergence corresponds to oscillations (in the absence of concentration effects) indicates that the new Hilbert space, in which $\mathbb{R}^{N n(\varepsilon)}$ is embedded, must capture these oscillations. In Section 3 we propose such a new extension by using a combination of two-scale convergence and Bloch-wave theory.

Remark 2.1.12. Let us briefly discuss the previous work of C. CONCA \& $\mathrm{M}$. VANNINATHAN [12] on the same problem. The key in comparing our results to theirs is to recognize that the spectral problem (7) is invariant upon rescaling by a factor 8 . In other words, by the change of variables

$$
y=\frac{x}{\varepsilon},
$$

the problem of finding all $\left(\lambda_{\eta}, u_{\eta}\right)$ solutions of

$$
\begin{aligned}
-\Delta u_{\eta} & =0 & & \text { in } \Omega_{\eta}^{*}, \\
\lambda_{\eta} \frac{\partial u_{\eta}}{\partial n} & =n \cdot \int_{\Gamma_{p}} u_{\eta} n d s & & \text { on } \Gamma_{p} \text { for } 1 \leqq p \leqq n(\eta), \\
u_{\eta} & =0 & & \text { on } \partial \Omega_{\eta}
\end{aligned}
$$

is equivalent to our spectral problem (7) with the definitions $n(\eta)=n(\varepsilon)$, $\Omega_{\eta}=\varepsilon^{-1} \Omega, \Omega_{\eta}^{*}=\varepsilon^{-1} \Omega_{\varepsilon}, \Gamma_{p}=\varepsilon^{-1} \Gamma_{p}^{\varepsilon}, Y_{p}=\varepsilon^{-1} Y_{p}^{\varepsilon}$ and the identities

$$
u_{\eta}(y)=u_{\varepsilon}(x), \quad \lambda_{\eta}=\lambda_{\varepsilon} .
$$


The above spectral problem (18) is exactly that studied in [12] by means of the Bloch-wave theory. It turns out that the resulting limit problem, defined in an unbounded domain, has nothing to do with the results obtained in this section by classical homogenization. Thus, another motivation of Section 3 is to understand this difference. As we see, we are able to recover the results of [12] by using our Bloch-wave homogenization method developed in Section 3 (see Remark 3.2.13).

\subsection{Convergence of the classical homogenization process}

This section is devoted to the proof of Theorem 2.1.1 concerning the strong convergence of the sequence of operators $\tilde{S}_{\varepsilon}$ defined by

$$
\begin{aligned}
\tilde{S_{\varepsilon}}: L^{2}(\Omega)^{N} & \rightarrow L^{2}(\Omega)^{N}, \\
s(x) & \rightarrow \widetilde{S_{\varepsilon}} s(x)=\sum_{p=1}^{n(\varepsilon)}\left(\frac{1}{\left|Y_{p}^{\varepsilon}\right|} \int_{\Gamma_{p}^{\varepsilon}} u_{\varepsilon} n d s\right) \chi_{Y_{p}^{\varepsilon}}(x),
\end{aligned}
$$

where $u_{\varepsilon}$ is the unique solution in $H^{1}\left(\Omega_{\varepsilon}\right)$ of

$$
\begin{aligned}
-\Delta u_{\varepsilon} & =0 & & \text { in } \Omega_{\varepsilon}, \\
\frac{\partial u_{\varepsilon}}{\partial n} & =\left(P_{\varepsilon} s\right) \cdot \boldsymbol{n} & & \text { on } \Gamma_{p}^{\varepsilon}, \text { for } 1 \leqq p \leqq n(\varepsilon), \\
u_{\varepsilon} & =0 & & \text { on } \partial \Omega,
\end{aligned}
$$

the right-hand side $P_{\varepsilon} s$ being defined by

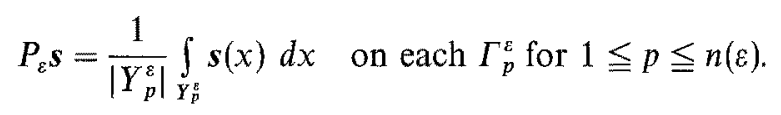

Since the definition (19) of $\tilde{S}_{\varepsilon}$ involves the solution $u_{\varepsilon}$ of the boundary-value problem (20), our first task is to homogenize this problem as $\varepsilon$ goes to zero. To do so, we use the two-scale convergence method (recently introduced in [2] and [23]), which is well-suited to the present periodic setting. It turns out that the determination of the limit of $\tilde{S}_{\varepsilon}$ (i.e., the weak convergence of $\tilde{S}_{\varepsilon}$ to its limit $S$ ) requires only a corrector result for $u_{\varepsilon}$, or equivalently the $\Gamma$-convergence of the energy associated with (20). Such results can also be obtained via standard homogenization procedures such as two-scale asymptotic expansions (see, e.g., [5,6,33]) rigorously justified by $G$ - or $H$-convergence (see, e.g., $[13,22,35,36]$ ). However, the strong convergence of the sequence $\widetilde{S}_{\varepsilon}$ seems to rely crucially on the two-scale convergence method (see the proof of Proposition 2.2.6). We begin by recalling the necessary results on two-scale convergence (we follow the notation of [2]).

Propositon 2.2.1. 1. Let $u_{\varepsilon}$ be a bounded sequence in $L^{2}(\Omega)$. There exist a subsequence (still denoted by $\varepsilon$ ) and a two-scale limit $u_{0}(x, y) \in L^{2}(\Omega \times Y)$ such that $u_{\varepsilon}(x)$ 
two-scale converges to $u_{0}(x, y)$ in the sense that

$$
\lim _{\varepsilon \rightarrow 0} \int_{\Omega} u_{\varepsilon}(x) \phi\left(x, \frac{x}{\varepsilon}\right) d x=\frac{1}{|Y|} \int_{\Omega} \int_{Y} u_{0}(x, y) \phi(x, y) d x d y
$$

for any function $\phi(x, y)$ in $\mathscr{D}\left[\Omega ; C_{\#}^{\infty}(Y)\right]$.

2. Let $u_{\varepsilon}$ be a bounded sequence in $H^{1}(\Omega)$. Then, up to a subsequence, $u_{\varepsilon}$ two-scale converges to a limit $u(x) \in H^{1}(\Omega)$, and $\nabla u_{\varepsilon}$ two-scale converges to $\nabla_{x} u(x)+\nabla_{y} u_{1}(x, y)$, where the function $u_{1}(x, y)$ belongs to $L^{2}\left[\Omega ; H_{\#}^{1}(Y) / \mathbb{R}\right]$.

3. Let $u_{\varepsilon}$ be a sequence of functions in $L^{2}(\Omega)$ which two-scale converges to a limit $u_{0}(x, y) \in L^{2}(\Omega \times Y)$. Assume further that

$$
\lim _{\varepsilon \rightarrow 0}\left\|u_{\varepsilon}\right\|_{L^{2}(\Omega)}^{2}=\frac{1}{|Y|}\left\|u_{0}\right\|_{L^{2}(\Omega \times Y)}^{2} .
$$

(Then $u_{\varepsilon}$ is said to two-scale converge strongly to its limit $u_{0}$.) For any sequence $v_{\varepsilon}$ which two-scale converges simply to a limit $v_{0}(x, y) \in L^{2}(\Omega \times Y)$,

$$
u_{\varepsilon}(x) v_{\varepsilon}(x) \rightarrow \frac{1}{|Y|} \int_{Y} u_{0}(x, y) v_{0}(x, y) d y \quad \text { in } L^{1}(\Omega) \text { weakly. }
$$

Since we are studying an homogenization problem in a perforated domain $\Omega_{\varepsilon}$, we use a well-known technical lemma [9] for extending the solution $u_{\varepsilon}$ of (20) to the whole limit domain $\Omega$. This allows us to study the convergence of the sequence $u_{\varepsilon}$ in the fixed space $H^{1}(\Omega)$. We remark in passing that two-scale convergence can handle homogenization problems in perforated domains without using any extension operator (see Section 2 in [2]), but the following extension lemma simplifies the presentation of the results.

Lemma 2.2.2. Let $\Omega_{\varepsilon}$ be a perforated domain defined by (3). There exists a bounded extension operator $X_{\varepsilon}$ acting from $H^{1}\left(\Omega_{\varepsilon}\right)$ into $H^{1}(\Omega)$ and a positive constant $C$ (independent of 8 ) such that

$$
X_{\varepsilon} v=v \quad \text { in } \Omega_{\varepsilon},\left\|X_{\varepsilon} v\right\|_{H^{1}(\Omega)} \leqq C\|v\|_{H^{1}(\Omega)}
$$

for any $v \in H^{1}\left(\Omega_{\varepsilon}\right)$.

To simplify the notation further, we denote by $u_{\varepsilon}$ both the solution of $(20)$ in $H^{1}\left(\Omega_{\varepsilon}\right)$ and its bounded extension $X_{\varepsilon} u_{\varepsilon}$ in $H^{1}(\Omega)$. The next step is to obtain a priori estimates for the solution $u_{\varepsilon}$ (which, by the way, provides a proof of Lemma 1.2.2).

Lemma 2.2.3. Let $u_{\varepsilon}$ be the unique solution of $(20)$ in $H^{1}\left(\Omega_{\varepsilon}\right)$ (extended to the whole domain $\Omega$ ). There exist two positive constants $c$ and $C$ independent of $\varepsilon$ and $s$ with $0<c \leqq C$ such that

$$
c\left\|P_{\varepsilon} s\right\|_{L^{2}(\Omega)} \leqq\left\|u_{\varepsilon}\right\|_{B^{1}(\Omega)} \leqq C\|s\|_{L^{2}(\Omega)}
$$


Proof. Multiplying (20) by $u_{\varepsilon}$ and integrating by parts yields

$$
\int_{\Omega_{\varepsilon}}\left|\nabla u_{\varepsilon}\right|^{2} d x=\sum_{p=1}^{n(\varepsilon)}\left(\frac{1}{\left|Y_{p}^{\varepsilon}\right|} \int_{Y_{p}^{\varepsilon}} s(x) d x\right) \cdot\left(\int_{\Gamma_{p}^{\varepsilon}} u_{\varepsilon} n d s\right) .
$$

Since the holes are isolated, we have

$$
\left|\int_{\Gamma_{P}^{\varepsilon}} u_{\varepsilon} \boldsymbol{n} d s\right|=\left|\int_{T_{P}^{\varepsilon}} \nabla u_{\varepsilon} d x\right| \leqq \varepsilon^{N / 2}\left\|\nabla u_{\varepsilon}\right\|_{L^{2}\left(T_{p}^{\varepsilon}\right)} .
$$

On the other hand, the Cauchy-Schwarz inequality gives

$$
\left|\frac{1}{\left|Y_{p}^{\varepsilon}\right|} \int_{Y_{p}^{\varepsilon}} s d x\right| \leqq \varepsilon^{-N / 2}\|\boldsymbol{s}\|_{L^{2}\left(X_{p}^{q}\right)} .
$$

Thus, the right-hand side of (21) is bounded by

$$
\sum_{p=1}^{n(\varepsilon)}\left\|\nabla u_{\varepsilon}\right\|_{L^{2}\left(Y_{p}^{E}\right)}\|\boldsymbol{s}\|_{L^{2}\left(Y_{p}^{p}\right)} \leqq\left\|\nabla u_{\varepsilon}\right\|_{L^{2}(\Omega)}\|\boldsymbol{s}\|_{L^{2}(\Omega)} .
$$

This gives an upper bound for the norm of $u_{\varepsilon}$. To get a lower bound, we multiply (20) by a test function $v_{\varepsilon}$ defined in each cell $Y_{p}^{\varepsilon}$, of center $\boldsymbol{x}_{p}$, by

$$
v_{\varepsilon}=-\left(P_{\varepsilon} s \cdot\left(\boldsymbol{x}-\boldsymbol{x}_{p}\right)\right) \theta\left(\frac{x}{\varepsilon}\right)
$$

where $\theta(y)$ is a $Y$-periodic function which is identically equal to 1 on the hole $T$ and vanishes on a neighborhood of $\partial Y$. Integrating by parts yields

$$
\int_{\Omega_{\varepsilon}} \nabla u_{\varepsilon} \cdot \nabla v_{\varepsilon} d x=\sum_{p=1}^{n(\varepsilon)}\left(P_{\varepsilon} s\right) \cdot\left(\int_{\Gamma_{p}^{\varepsilon}} v_{\varepsilon} n d s\right) .
$$

The right-hand side of (22) is easily seen to be equal to $|T| \varepsilon^{N} \sum_{p=1}^{n(\varepsilon)}\left|P_{\varepsilon} s\right|^{2}$, while its left-hand side can be estimated by remarking that $v_{\varepsilon}$ is uniformly bounded in $H^{1}(\Omega)$, giving the lower bound for the norm of $u_{\varepsilon}$.

We are now in a position to give the homogenized system for (20).

Proposition 2.2.4. The sequence $u_{\varepsilon}$ of solutions of (20) (extended to the whole domain $\Omega)$ converges weakly in $H_{0}^{1}(\Omega)$ to the unique solution $u$ of the homogenized problem

$$
\begin{aligned}
-\operatorname{div}(A \nabla u) & =\operatorname{div}((I-A) s) & & \text { in } \Omega, \\
u & =0 & & \text { on } \partial \Omega,
\end{aligned}
$$

where the matrix $A$ is defined by $A_{i j}=\int_{Y^{*}}\left(\nabla_{y} w_{i}+e_{i}\right) \cdot\left(\nabla_{y} w_{j}+e_{j}\right) d y$, and where $\left(w_{i}\right)_{1 \leqq i \leqq N}$ is the family of solutions of the cell problems (16). Furthermore, $\nabla u_{\varepsilon}$ 
two-scale converges to $\nabla_{x} u(x)+\nabla_{y} u_{1}(x, y)$, with

$$
u_{1}(x, y)=\sum_{i=1}^{N} w_{i}(y)\left(\frac{\partial u}{\partial x_{i}}(x)-s_{i}(x)\right)
$$

and the energies converge:

$$
\lim _{\varepsilon \rightarrow 0} \int_{\Omega_{\varepsilon}}\left|\nabla u_{\varepsilon}\right|^{2} d x=\int_{\Omega} \int_{Y^{*}}\left|\nabla_{x} u+\nabla_{y} u_{1}(x, y)\right|^{2} d x d y
$$

Proof. We know from Lemma 2.2.3 that the sequence $u_{\varepsilon}$ is bounded in $H_{0}^{1}(\Omega)$. By Proposition 2.2.1, up to a subsequence, $u_{\varepsilon}$ two-scale converges to a limit $u(x)$ (which, of course, coincides with the usual weak $H_{0}^{1}(\Omega)$ limit), and $\nabla u_{\varepsilon}$ two-scale converges to $\nabla u(x)+\nabla_{y} u_{1}(x, y)$ where $u_{1} \in L^{2}\left[\Omega ; H_{* 4}^{1}(Y)\right]$. Then, we multiply (20) by a test function $\phi(x)+\varepsilon \phi_{1}(x, x / \varepsilon)$, with $\phi \in \mathscr{D}(\Omega)$ and $\phi_{1} \in \mathscr{D}\left[\Omega ; C_{\#}^{\infty}(Y)\right]$. Integrating by parts, we obtain

$$
\begin{array}{r}
\int_{\Omega} \chi\left(\frac{x}{c}\right) \nabla u_{\varepsilon} \cdot\left(\nabla \phi(x)+\nabla_{y} \phi_{1}\left(x, \frac{x}{\varepsilon}\right)+\varepsilon \nabla_{x} \phi_{1}\left(x, \frac{x}{\varepsilon}\right)\right) d x \\
=\sum_{p=1}^{n(\varepsilon)}\left(\frac{1}{\left|Y_{p}^{\varepsilon}\right|} \int_{Y_{p}^{p}} s(x) d x\right) \cdot\left(\int_{r_{p}^{\varepsilon}}\left(\phi(x)+\varepsilon \phi_{1}\left(x, \frac{x}{\varepsilon}\right)\right) n d s\right),
\end{array}
$$

where $\chi(y)$ is the characteristic function of $Y^{*}$. Green's formula in each hole $T_{p}^{\varepsilon}$ gives

$$
\int_{\Gamma_{p}^{z}}\left(\phi(x)+\varepsilon \phi_{1}\left(x, \frac{x}{\varepsilon}\right)\right) n d s=-\int_{T_{y}^{\varepsilon}}\left(\nabla \phi(x)+\nabla_{y} \phi_{1}\left(x, \frac{x}{\varepsilon}\right)+\varepsilon \nabla_{x} \phi_{1}\left(x, \frac{x}{\varepsilon}\right)\right) d x .
$$

Therefore, if we use the definitions (10) and (11) of $P_{\varepsilon}$ and $E_{\varepsilon}$, the right-hand side of (25) becomes

$$
\int_{\Omega}\left(\chi\left(\frac{x}{\varepsilon}\right)-1\right) E_{\varepsilon} P_{\varepsilon} s \cdot\left(\nabla \phi(x)+\nabla_{y} \phi_{1}\left(x, \frac{x}{\varepsilon}\right)+\varepsilon \nabla_{x} \phi_{1}\left(x, \frac{x}{\varepsilon}\right)\right) d x .
$$

Since the size of the cells goes to zero as $\varepsilon$ does, it is easy to prove (we leave this to the reader) that

$$
E_{\varepsilon} P_{\varepsilon} s \rightarrow s \text { in } L^{2}(\Omega)^{N} \text { strongly for each } s \in L^{2}(\Omega)^{N} .
$$

Thus, we can easily pass to the limit in (27). On the other hand, passing to the two-scale limit (by Proposition 2.2.1) in the left-hand side of (25), we obtain

$$
\int_{\Omega} \int_{Y^{*}}\left(\nabla u(x)+\nabla_{y} u_{1}(x, y)\right) \cdot\left(\nabla \phi(x)+\nabla_{y} \phi_{1}(x, y)\right) d x d y=
$$




$$
\begin{aligned}
& =-\int_{\Omega} \int_{T} s \cdot\left(\nabla \phi(x)+\nabla_{y} \phi_{1}(x, y)\right) d x d y \\
& =|T| \int_{\Omega} \phi(x) \operatorname{div}_{x} s d x+\int_{\Omega} \int_{\partial T} s(x) \cdot n_{y} \phi_{1}(x, y) d x d s .
\end{aligned}
$$

Equation (29) is nothing else but a variational formulation for $\left(u, u_{1}\right)$ in the space $H_{0}^{1}(\Omega) \times L^{2}\left[\Omega ; H_{\# \neq}^{1}\left(Y^{*}\right) / \mathbb{R}\right]$. By application of the Lax-Milgram lemma, it is easily seen that (29) admits a unique solution. Thus the entire sequence $u_{\varepsilon}$ converges to its limit $u$. From (29) we derive the associated system of equations (the so-called two-scale homogenized problem)

$$
\begin{array}{ll}
-\Delta_{y} u_{1}(x, y)=0 & \text { in } \Omega \times Y^{*}, \\
-\operatorname{div}_{x}\left(\int_{Y^{*}}\left(\nabla u(x)+\nabla_{y} u_{1}(x, y)\right) d y\right)=|T| \operatorname{div}_{x} s & \text { in } \Omega, \\
u(x)=0 & \text { on } \partial \Omega . \\
\left(\nabla u(x)+\nabla_{y} u_{1}(x, y)-s(x)\right) \cdot \boldsymbol{n}_{y}=0 & \text { on } \partial T \times \Omega, \\
y \rightarrow u_{1}(x, y) \text { is } Y \text {-periodic. } &
\end{array}
$$

By linearity, the solution $u_{1}$ in (30) can be computed in terms of $\nabla u(x)$ and of the solutions $\left(w_{i}\right)_{1 \leqq i \leqq N}$ of the cell problems (16):

$$
u_{1}(x, y)=\sum_{i=1}^{N} w_{i}(y)\left(\frac{\partial u}{\partial x_{i}}(x)-s_{i}(x)\right)
$$

Finally, eliminating the $y$ variable in (30) yields the homogenized equation (23) (for details, see Section 2 in [2]) by remarking that

$$
\begin{gathered}
\int_{Y^{*}}\left(\nabla u(x)+\nabla_{y} u_{1}(x, y)\right) d y=\theta \nabla u(x)+\sum_{i=1}^{N}\left(\frac{\partial u}{\partial x_{i}}(x)-s_{i}(x)\right) \int_{Y^{*}} \nabla w_{i}(y) d y, \\
\int_{Y^{*}} \nabla w_{i}(y) d y=(A-\theta I) e_{i},
\end{gathered}
$$

where the matrix $A$ has been defined in (15). To prove the remaining statement (24), we multiply (20) by $u_{\varepsilon}$, and integrate the product by parts:

$$
\begin{aligned}
\int_{\Omega_{\varepsilon}}\left|\nabla u_{\varepsilon}\right|^{2} d x & =\sum_{p=1}^{n(\varepsilon)}\left(\frac{1}{\left|Y_{p}^{\varepsilon}\right|} \int_{Y_{\delta}^{\varepsilon}} s(x) d x\right) \cdot\left(\int_{\Gamma_{p}^{\varepsilon}} u_{\varepsilon} n d s\right) \\
& =\int_{\Omega}\left(\chi\left(\frac{x}{\varepsilon}\right)-1\right) E_{\varepsilon} P_{\varepsilon} s \cdot \nabla u_{\varepsilon} d x .
\end{aligned}
$$


To pass to the limit in (33), we recall that $E_{\varepsilon} P_{\varepsilon} s$ converges strongly to $s$ in $L^{2}(\Omega)^{N}$ (see (28)). Thus the limit of (33) is the same as that of

$$
\int_{\Omega}\left(\chi\left(\frac{x}{\varepsilon}\right)-1\right) s \cdot \nabla u_{\varepsilon} d x
$$

By definition of two-scale convergence, the limit of (34) is simply

$$
-\int_{\Omega} \int_{T} s \cdot\left(\nabla u(x)+\nabla_{y} u_{1}(x, y)\right) d x d y=\int_{\Omega} \int_{Y^{*}}\left|\nabla_{x} u+\nabla_{y} u_{1}(x, y)\right|^{2} d x d y,
$$

thanks to an easy integration by parts in (30).

With the help of the above homogenization result, we can compute the limit operator $S$.

Proposition 2.2.5. The sequence $\widetilde{S}_{\varepsilon}$ converges weakly in $L^{2}(\Omega)^{N}$ to its limit $S$ defined by

$$
S s=(A-I) \nabla u-(A-\theta I) s,
$$

where $\theta=\left|Y^{*}\right|$, and $u$ is the unique solution in $H^{1}(\Omega)$ of the homogenized problem (23).

Proof. We study the convergence of $\widetilde{S}_{\varepsilon}$ by inspecting the limit of $\int_{\Omega} \tilde{S}_{\varepsilon} s \cdot s d x$. From the energy relation (33) we deduce that

$$
\int_{\Omega} \tilde{S}_{\varepsilon} s \cdot s d x=\int_{\Omega_{\varepsilon}} \nabla u_{\varepsilon} \cdot \nabla u_{\varepsilon} d x .
$$

We have just computed the limit of the right-hand side of (36) (see (24)) which defines a continuous, self-adjoint, linear operator $S$ acting on $L^{2}(\Omega)^{N}$ by

$$
\int_{\Omega} S \boldsymbol{s} \cdot \boldsymbol{s} d x=\int_{\Omega} \int_{Y^{*}}\left|\nabla u+\nabla_{y} u_{1}(x, y)\right|^{2} d x d y
$$

where $\left(u(x), u_{1}(x, y)\right)$ is the solution of the two-scale homogenized problem (30). Replacing $u_{1}$ by its value (31) and using the relationship (32) yield

$$
S \mathbf{s}=(A-I) \nabla u-(A-\theta I) \mathbf{s} .
$$

To complete the proof of Theorem 2.1.1, it remains to establish.

Proposition 2.2.6. The sequence $\tilde{S}_{\varepsilon}$ converges strongly in $L^{2}(\Omega)^{N}$ to its limit $S$.

Proof. Let $\boldsymbol{t}_{\varepsilon}$ be any sequence which converges weakly to a function $\boldsymbol{t}$ in $L^{2}(\Omega)^{N}$. We need to show that

$$
\lim _{\varepsilon \rightarrow 0} \int_{\Omega} S_{\varepsilon} s \cdot t_{\varepsilon} d x=\int_{\Omega} \boldsymbol{S} \boldsymbol{s} \cdot \boldsymbol{t} d x
$$


By introducing a function $v_{\varepsilon}$, defined as the unique solution in $H^{1}\left(\Omega_{\varepsilon}\right)$ of

$$
\begin{aligned}
-\Delta v_{\varepsilon} & =0 & & \text { in } \Omega_{\varepsilon}, \\
\frac{\partial v_{\varepsilon}}{\partial n} & =\left(P_{\varepsilon} \boldsymbol{t}_{\varepsilon}\right) \cdot \boldsymbol{n} & & \text { on } \Gamma_{p}^{\varepsilon} \text { for } 1 \leqq p \leqq n(\varepsilon), \\
v_{\varepsilon} & =0 & & \text { on } \partial \boldsymbol{\Omega},
\end{aligned}
$$

we easily obtain

$$
\int_{\Omega} \tilde{S_{\varepsilon}} \boldsymbol{s} \cdot \boldsymbol{t}_{\varepsilon} d x=\int_{\Omega_{\varepsilon}} \nabla u_{\varepsilon} \cdot \nabla v_{\varepsilon} d x
$$

To pass to the limit in (39) we again use two-scale convergence. To this end, we need first to homogenize equation (38) for $v_{\varepsilon}$. Note that, thanks to the a priori estimate in Lemma $2.2 .3, v_{\varepsilon}$ is also uniformly bounded in $H_{0}^{1}(\Omega)$. Thus, the homogenization of (38) follows the same procedure as that in Proposition 2.2.4. The only difference comes from the argument used to pass to the limit in the right-hand side of the variational formulation (25):

$$
\begin{aligned}
& \sum_{p=1}^{n(\varepsilon)}\left(\frac{1}{\left|Y_{p}^{\varepsilon}\right|} \int_{Y_{p}^{\varepsilon}} t_{\varepsilon}(x) d x\right) \cdot\left(\int_{\Gamma_{p}^{\varepsilon}}\left(\phi(x)+\varepsilon \phi_{1}\left(x, \frac{x}{\varepsilon}\right)\right) n d s\right) \\
& \quad=\int_{\Omega}\left(\chi\left(\frac{x}{\varepsilon}\right)-1\right) E_{\varepsilon} P_{\varepsilon} t_{\varepsilon} \cdot\left(\nabla \phi(x)+\nabla_{y} \phi_{1}\left(x, \frac{x}{\varepsilon}\right)+\varepsilon \nabla_{x} \phi_{1}\left(x, \frac{x}{\varepsilon}\right)\right) d x \\
& =\int_{\Omega} t_{\varepsilon} \cdot E_{\varepsilon} P_{\varepsilon}\left(\left(\chi\left(\frac{x}{\varepsilon}\right)-1\right)\left(\nabla \phi(x)+\nabla_{y} \phi_{1}\left(x, \frac{x}{\varepsilon}\right)+\varepsilon \nabla_{x} \phi_{1}\left(x, \frac{x}{\varepsilon}\right)\right)\right) d x
\end{aligned}
$$

It is not difficult to check that, for any smooth function $\psi(x, y) \in \mathscr{D}\left(\Omega ; C_{\#}^{\infty}(Y)\right)$, $E_{\varepsilon} P_{\varepsilon} \psi(x, x / \varepsilon)$ converges strongly to $\int_{Y} \psi(x, y) d y$ in $L^{2}(\Omega)$. With this property, it is now straightforward to pass to the limit in (40). Denoting by $v(x)$ the weak limit of $v_{\varepsilon}$, and by $v_{1}(x, y)$ the function such that $\nabla v_{\varepsilon}$ two-scale converges to $\nabla_{x} v+\nabla_{y} v_{1}$, we find that the sequence $v_{\varepsilon}$ converges in the space $H_{0}^{1}(\Omega) \times L^{2}\left[\Omega ; H_{\#}^{1}\left(Y^{*}\right) / \mathbb{R}\right]$ to the unique solution $\left(v, v_{1}\right)$ of the same homogenized problem $(30)$ where the right-hand side $s$ is simply changed for $t$.

Then, to pass to the limit in (39) we use the so-called strong two-scale convergence of $\nabla u_{\varepsilon}$ (see part 3 of Proposition 2.2.1), which is a consequence of the energy convergence (24). Finally, we obtain

$$
\lim _{\varepsilon \rightarrow 0} \int_{\Omega_{\varepsilon}} \nabla u_{\varepsilon} \cdot \nabla v_{\varepsilon} d x=\iint_{\Omega}\left(\nabla_{x} u+\nabla_{y} u_{1}\right) \cdot\left(\nabla_{x} v+\nabla_{y} v_{1}\right) d x d y
$$


which gives the desired result up to an easy integration by parts in the homogenized system (30).

Remark 2.2.7. In the definition of the original operator $S_{\varepsilon}$, we asked that each tube displacement $s_{p}$ be constant on its section $T_{p}$. From a physical point of view, this ensures that the tube displacements are rigid. To define a proper extension $\tilde{S}_{\varepsilon}=E_{\varepsilon} S_{\varepsilon} P_{\varepsilon}$ of $S_{\varepsilon}$, it is absolutely necessary to define a projection operator $P_{\varepsilon}$ which maps $L^{2}(\Omega)^{N}$ onto piecewise constant functions on each tube boundary, in order to preserve this requirement of the model. Previous attempts to homogenize system (20) have failed because the violation of this property leads to a different homogenized problem and an unphysical limit operator. For example, our results do not agree with those in [28] because uncorrect asymptotic expansions were used that lead to the implicit boundary condition

$$
\frac{\partial u_{\varepsilon}}{\partial n}=s(x) \cdot n \quad \text { on } \Gamma_{p}^{\varepsilon}
$$

which contradicts the model's requirement of rigid displacements.

\subsection{Spectrum of the limit operator}

This section is devoted to the study of the spectrum $\sigma(S)$ of the limit operator $S$, i.e., to the proofs of Theorems 2.1.4, 2.1.5, and Proposition 2.1.11. Recall that the limit operator $S$ is defined by

$$
S s=(A-I) \nabla u-(A-\theta I) s,
$$

where $u$ is the unique solution in $H^{1}(\Omega)$ of the homogenized problem

$$
\begin{aligned}
-\operatorname{div}(A \nabla u) & =\operatorname{div}((I-A) s) & & \text { in } \Omega, \\
u & =0 & & \text { on } \partial \Omega,
\end{aligned}
$$

and $A$ is the constant homogenized matrix defined by (15). Since $A$ is symmetric, and thus diagonizable, we may assume with no loss of generality, that $A$ is diagonal in the canonical basis $\left(e_{i}\right)_{1 \leqq i \leqq N}$, with eigenvalues $0<\alpha_{1} \leqq \cdots \leqq \alpha_{N} \leqq \theta$.

A generic eigenvalue $\lambda$ and eigenvector $s$ of $S$ satisfy

$$
S s=\lambda s
$$

which, combined with (41), implies that the corresponding potential $u$ is given by

$$
\nabla u=(A-I)^{-1}(A+(\lambda-\theta) I) \mathbf{s}
$$

Introducing a matrix $B(\lambda)$ defined by

$$
B(\lambda)=A-(I-A)(A+(\lambda-\theta) I)^{-1}(I-A),
$$


we find that the potential $u$ must be a solution in $H_{0}^{1}(\Omega)$ of

$$
\begin{aligned}
-\operatorname{div} B(\lambda) \nabla u=0 & \text { in } \Omega, \\
u=0 & \text { on } \partial \Omega .
\end{aligned}
$$

Note that (44) is either elliptic or hyperbolic (but always with a Dirichlet boundary condition for all variables) and that $B(\lambda)$ may have eigenvalues equal to infinity. Investigating the possible solutions of (44) should give us a characterization of the eigenvalues of $S$. However, since $S$ is not compact, its spectrum is not merely made of eigenvalues, but can also contain an essential part. To characterize the essential spectrum of $S$ we use Weyl's criterion (see, e.g., [31]).

Lemma 2.3.1. A real $\lambda$ belongs to the essential spectrum of $S$ if and only if there exists a sequence $s_{n} \in L^{2}(\Omega)^{N}$ such that

$$
\begin{aligned}
s_{n} \rightarrow 0 & \text { in } L^{2}(\Omega)^{N} \text { weakly, with }\left\|s_{n}\right\|_{L^{2}(\Omega)^{N}}=1 \\
S s_{n}-\lambda s_{n} \rightarrow 0 & \text { in } L^{2}(\Omega)^{N} \text { strongly. }
\end{aligned}
$$

Proposition 2.3.2. Let $\lambda=\theta-\alpha_{i}$ for some $i \in\{1, \ldots, N\}$. In this case $B(\lambda)$ has an eigenvalue equal to infinity. Then $\lambda$ is an eigenvalue of infinite multiplicity of $S$.

Proof. By multiplying equation (43) by the eigenvector $\boldsymbol{e}_{i}$ and integrating against any test function on $\Omega$, we obtain

$$
\frac{\partial u}{\partial x_{i}}(x)=0 \quad \text { in } \Omega .
$$

Thanks to the Dirichlet boundary condition on $\partial \Omega$, this implies that $u$ is identically zero in $\Omega$. Thus, (41) and (42) yield

$$
\begin{aligned}
\left(A-\alpha_{i} I\right) s=0 & \text { in } \Omega, \\
\operatorname{div}(I-A) s=0 & \text { in } \Omega,
\end{aligned}
$$

which gives the definition of the associated eigenspace in $L^{2}(\Omega)^{N}$, which is infinitedimensional, as can easily be checked by taking

$$
s(x)=\phi\left(x_{1}, \ldots, x_{i-1}, x_{i+1}, \ldots, x_{N}\right) e_{i},
$$

where the scalar function $\phi$ does not depend on $x_{i}$.

Proposition 2.3.3. Let $\lambda=\theta+\left(1-2 \alpha_{i}\right) / \alpha_{i}$ for some $i \in\{1, \ldots, N\}$. In this case $B(\lambda)$ has a zero eigenvalue. Then $\lambda$ belongs to the essential spectrum of $S$.

Proof. In the isotropic case $\left(\alpha_{i}=\alpha\right.$ for all $\left.i \in\{1, \ldots, N\}\right)$, one can easily prove that $\lambda$ is an eigenvalue of infinite multiplicity whose eigenspace is $\langle\nabla q$ such that $\left.q \in H_{0}^{1}(\Omega)\right\}$. In the anisotropic case, it is generally not true that $\lambda$ is an eigenvalue. For example, in two dimensions with $\alpha_{1}<\alpha_{2}$, if we assume with no loss of 
generality, that $\lambda=\theta+\left(1-2 \alpha_{1}\right) / \alpha_{1}$, then the associated potential $u$ satisfies $\partial^{2} u / \partial x_{2}^{2}=0$, which, together with the Dirichlet boundary condition, implies that $u$ is identically zero in $\Omega$. Then, by (43), $s$ must also be equal to zero, which means that $\lambda$ is not an eigenvalue.

Thus, we apply Weyl's criterion to show that $\lambda$ belongs to $\sigma(S)$. Let $\phi(y)$ be a $Y$-periodic function defined by

$$
\phi(y)= \begin{cases}y_{i} & \text { if } 0<y_{i}<\frac{1}{2} \\ 1-y_{i} & \text { if } \frac{1}{2} \leqq y_{i}<1\end{cases}
$$

Let us check Weyl's criterion for the sequence $s_{n}$ defined by

$$
s_{n}(x)=\nabla\left(n^{-1} \phi(n x)\right) \text {. }
$$

The function $s_{n}$ takes alternatively the values $e_{i}$ and $-e_{i}$ with periodicity $n^{-1}$, where $e_{i}$ is the eigenvector associated with the eigenvalue $\alpha_{i}$. It is easily seen that $\boldsymbol{s}_{n}$ converges weakly to zero in $L^{2}(\Omega)^{N}$, while its norm is uniformly bounded away from zero. To show that $S s_{n}-\lambda s_{n}$ converges strongly to zero, we must first compute the associated potential $u_{n}$, the solution in $H_{0}^{1}(\Omega)$ of

$$
-\operatorname{div} A \nabla u_{n}=\operatorname{div}(I-A) s_{n} .
$$

Studying the asymptotic behavior of the solution of $u_{n}(45)$ is again a problem of homogenization. Then, it is a classical matter to prove (for example, by using two-scale convergence) that

$$
u_{n}(x)=-\frac{1-\alpha_{i}}{\alpha_{i}} n^{-1} \phi(n x)+r_{n}(x)
$$

where $r_{n}$ is a remainder term which converges strongly to zero in $H^{1}(\Omega)$. Thus,

$$
S \mathbf{s}_{n}-\lambda \mathbf{s}_{n}=(A-I) \nabla r_{n},
$$

which proves the desired result.

Proposition 2.3.4. Let $\lambda$ be such that the matrix $B(\lambda)$ is either positive-definite, or negative-definite. Then, $\lambda$ does not belong to $\sigma(S)$.

Proof. It is easily seen from (44) that the potential $u$ must be zero. Then, from (43) we deduce that $s$ is also zero. Thus $\lambda$ is not an eigenvalue of $S$. To show that $\lambda$ does not even belong to the essential spectrum of $S$, we try to get a contradiction from Weyl's criterion. Assume that there exists a normalized sequence $s_{n} \in L^{2}(\Omega)^{N}$ such that

$$
s_{n} \rightarrow 0 \text { weakly, } \quad r_{n}=S s_{n}-\lambda s_{n} \rightarrow 0 \text { strongly. }
$$

From the homogenized equation we get

$$
-\operatorname{div} B(\lambda) \nabla u_{n}=-\operatorname{div}(I-A)(A+(\lambda-\theta) I)^{-1} r_{n} .
$$


From standard regularity results for this elliptic equation, we infer that $u_{n}$ is compact in $H^{1}(\Omega)$, which is in contradiction with the fact that $s_{n}$ cannot converge strongly, since

$$
s_{n}=(A+(\lambda-\theta) I)^{-1}\left((A-I) \nabla u_{n}-r_{n}\right) .
$$

Proposition 2.3.5. Let $\lambda$ be such that $B(\lambda)$ has neither a zero nor an infinite eigenvalues, but has simultaneously positive and negative eigenvalues. Then, $\lambda$ belongs to the essential spectrum of $S$.

Proof. In this case, the spectral equation (44) is hyperbolic: we do not know if it has a solution in general. (For some special choices of $\Omega$ and $B$, one can show that there exist an infinite number of solutions.) Thus, we do not try to prove that $\lambda$ is an eigenvalue; rather, we again apply Weyl's criterion to a suitably chosen sequence $s_{n} \in L^{2}(\Omega)^{N}$. We assume that the matrix $B(\lambda)$ has two non-zero eigenvalues $\beta_{1}^{2}$ and $-\beta_{N}^{2}$ such that $\beta_{1}$ and $\beta_{N}$ are positive reals. Let us consider in the rectangular cell $Z=\left(0, \beta_{1}\right) \times(0,1) \times \cdots \times(0,1) \times\left(0, \beta_{N}\right)$ the hyperbolic equation

$$
\begin{gathered}
-\operatorname{div}_{y}\left(B(\lambda) \nabla_{y} w\right)=0 \text { in } Z, \\
y \rightarrow w(y) \text { is } Z \text {-periodic. }
\end{gathered}
$$

As is well known, (46) has infinitely many solutions. From among them, we choose

$$
w(y)=\phi_{+}\left(\beta_{1}^{-1} y_{1}+\beta_{N}^{-1} y_{N}\right)+\phi_{-}\left(\beta_{1}^{-1} y_{1}-\beta_{N}^{-1} y_{N}\right)
$$

where $\phi_{+}$and $\phi_{-}$are two 1-periodic functions of a single real variable. Then, from (43) we define $s_{n}$ by

$$
\boldsymbol{s}_{n}(x)=(A+(\lambda-\theta) I)^{-1}(A-I) \nabla\left(n^{-1} w(n x)\right) .
$$

It is not difficult to check that $s_{n}$ converges weakly to zero in $L^{2}(\Omega)^{N}$, while its norm is uniformly bounded away from zero. As in Proposition 2.3.3, we must compute the associated potential $u_{n}$ to show that $S s_{n}-\lambda s_{n}$ converges strongly to zero. A standard homogenization result implies that

$$
u_{n}(x)=n^{-1} w(n x)+r_{n}(x)
$$

where $r_{n}$ is a remainder term which converges strongly to zero in $H^{1}(\Omega)$. Thus, we have

$$
S s_{n}-\lambda s_{n}=(A-I) \nabla r_{n},
$$

which proves the desired result.

To conclude this section, it remains to prove Proposition 2.1 .11 on the pointwise convergence of the spectra of a sequence of strongly convergent operators.

Proof of Proposition 2.1.11. Let $\lambda \in \sigma(S)$, and assume that $\lambda$ is not the limit of any sequences of eigenvalues of $S_{\varepsilon}$. This means that there exists a positive constant $\delta>0$ such that

$$
\left|\lambda_{\varepsilon}-\lambda\right| \geqq \delta
$$


for sufficiently small $\varepsilon$ and for any eigenvalue $\lambda_{\varepsilon} \in \sigma\left(S_{\varepsilon}\right)$. Obviously this implies that

$$
\left\|\tilde{S}_{\varepsilon} s-\lambda \boldsymbol{s}\right\|_{L^{2}(\Omega)^{N}} \geqq \delta
$$

for any function $s(x) \in L^{2}(\Omega)^{N}$. Since the convergence of $S_{\varepsilon}$ to $S$ is strong, one can pass to the limit in (47) and obtain

$$
\|S \boldsymbol{s}-\lambda \boldsymbol{s}\|_{L^{2}(\Omega)^{n}} \geqq \delta,
$$

for any function $s(x)$, which contradicts the fact that $\lambda$ belongs to the spectrum of $S$. Thus, $\lambda$ is attained as a limit of a sequence $\lambda_{\varepsilon} \in \sigma\left(S_{\varepsilon}\right)$.

To complete the proof of Proposition 2.1.11, it remains to show that if a sequence of eigenvalues $\lambda_{\varepsilon}$ converges to a limit $\lambda$ outside $\sigma(S)$, then any associated sequence of eigenvectors $s_{\varepsilon}(x)$ converges to zero weakly in $L^{2}(\Omega)^{N}$. The spectral equation is

$$
\tilde{S}_{\varepsilon} s_{\varepsilon}=\lambda_{\varepsilon} s_{\varepsilon} .
$$

Thanks to the strong convergence of $\widetilde{S}_{\varepsilon}$, we can pass to the limit (up to a subsequence) in (48), and denoting by $s$ the weak limit of the sequence $s_{\varepsilon}$ we obtain

$$
S s=\lambda s
$$

Since $\lambda$ does not belong to $\sigma(S)$, this necessarily implies that the limit $s$ is equal to zero.

\section{Bloch-wave homogenization: a coupled macro-microscopic limit operator}

\subsection{Motivation and discrete Bloch waves}

As we already discussed at the end of Section 2.1, the convergence analysis of the operator $\widetilde{S}_{\varepsilon}$ in $L^{2}(\Omega)^{N}$ is not entirely satisfactory. Indeed we proved the convergence of the spectral family of $\tilde{S}_{\varepsilon}$ to that of the limit operator $S$, which only gives a very weak convergence of the corresponding spectra. We recall that any element of the spectrum of $S$ is attained by a sequence of eigenvalues of $\tilde{S}_{\varepsilon}$ as $\varepsilon$ goes to zero. However, it may happen that some sequence of eigenvalues of $\widetilde{S}_{\varepsilon}$ converges to a limit which does not belong to the spectrum of $S$. In this case, by virtue of Proposition 2.1.11, the corresponding sequence of normalized eigenvectors converges weakly to zero in $L^{2}(\Omega)^{N}$.

This indicates that the above lack of continuity of the spectrum of $\tilde{S}_{\varepsilon}$ as $\varepsilon$ goes to zero is due to the particular choice of $\tilde{S}_{\varepsilon}$ as an extension of the finite-dimensional operator $S_{\varepsilon}$. Recall that $S_{\varepsilon}$ was originally defined on $\mathbb{R}^{N n(\varepsilon)}$, which was embedded in $L^{2}(\Omega)^{N}$ to define its extension $\widetilde{S}_{\varepsilon}$ on a fixed Hilbert space independent of $c$. Although "natural", this choice of $L^{2}(\Omega)^{N}$ is somewhat arbitrary, and is actually the cause of our troubles. In other words, we can select a different extension of $S_{\varepsilon}$ and a different reference space in which $\mathbb{R}^{N n(\varepsilon)}$ is embedded that yield a different limit operator having a much larger spectrum. Of course, the question is how to find such an extension. In our quest we should be guided by the fact that the eigenvalues 
of $\widetilde{S}_{\varepsilon}$ which "escape" from the spectrum of $S$ correspond to eigenvectors which converge weakly to zero in $L^{2}(\Omega)^{N}$. Thus, our new choice of extension should "capture" the oscillations contained in these weak convergences. In particular, eigenvectors of $\widetilde{S}_{\varepsilon}$ have a tendency to oscillate on the scale $\varepsilon$ of the periodic arrangement of the tubes. A natural candidate for the reference space is that of periodically oscillating functions $\varphi(x, x / \varepsilon)$, where $\varphi(x, y)$ is periodic in $y$ for all $x$ in $\Omega$.

From a physical point of view it is also clear that the macroscopic limit operator $S$ cannot contain all the limit eigenfrequencies of $S_{\varepsilon}$. As mentioned in Remark 2.1.6, the spectrum of $S$ includes only those frequencies corresponding to a macroscopic displacement of the tubes. More precisely, the smoothness of the associated eigenvectors (which belong to $\left.L^{2}(\Omega)^{N}\right)$ implies that neighboring tubes have similar displacements. In particular, this does not take into account the physically reasonable situation where two adjacent tubes vibrate with opposite phases (their displacements take opposite values). This phenomenon suggests that we consider a period containing a bundle of tubes having uncorrelated vibrations rather than a single tube as we did in Section 2.

Having this in mind, we now regard the fluid domain $\Omega_{\varepsilon}$, defined in Section 1.2, as a periodic domain with a new period $\varepsilon K$ corresponding to a new reference cell $K Y=(0 ; K)^{N}$. (Here $K$ is a given positive integer.) In the reference cell $K Y$ there are $K^{N}$ tubes $\left(T_{j}\right)_{0 \leqq j \leqq K-1}$ indexed by a multi-integer $j=\left(j_{1}, \ldots, j_{N}\right)$, where each component belongs to $\{0,1, \ldots, K-1\}$. To each tube $T_{j}$ in the periodic reference cell $K Y$, we associate the subcell $Y_{j}$ and the fluid subcell $Y_{j}^{*}=Y_{j} \backslash T_{j}$ analogous to $Y$ and $Y^{*}$ respectively (see Figure 3).

The main idea of this new framework is to attach to each tube $T_{j}$ in the reference cell $K Y$ a different displacement function $s_{j}(x)$. This procedure allows us to extend the original finite-dimensional operator $S_{\varepsilon}$ to an operator $S_{\varepsilon}^{K}$ acting on $\left[L^{2}(\Omega)^{N}\right]^{K^{N}}$ (instead of $L^{2}(\Omega)^{N}$ for $\widetilde{S}_{\varepsilon}$ ). We emphasize that the family $\left(s_{j}(x)\right)_{0 \leqq j \leqq K-1}$ is equivalent to a single oscillating displacement $s(x, y) \in L^{2}(\Omega \times K Y)^{N}$ which is

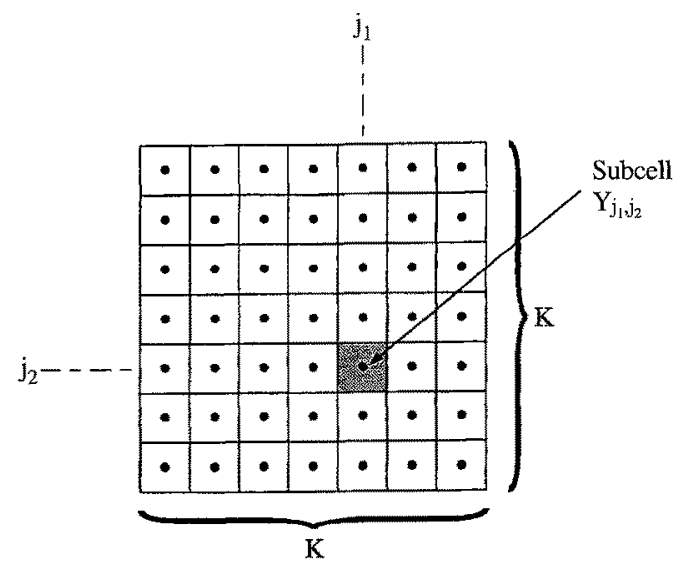

Fig. 3. Reference cell $K Y$ composed of $K \times K$ subcells $\left(Y_{j_{1}, j_{2}}\right)_{\substack{0 \leqq j \\ 0 \leq j_{2}} \underset{K}{K}-1}$ (in 2 dimensions) 
constant and equal to $s_{j}(x)$ in each subcell $Y_{j}$. (In other words, the space $\left[L^{2}(\Omega)^{N}\right]^{K^{N}}$ is isomorphic to the subspace of $L^{2}(\Omega \times K Y)^{N}$ consisting of piecewise constant functions in $y$.) Thus, according to our intuition, the new operator $S_{\varepsilon}^{K}$ should capture in the limit more oscillating eigenvectors than $\widetilde{S}_{\varepsilon}$.

To give a precise definition of this operator $S_{\varepsilon}^{K}$, we have to introduce, as in Section 2.1, two linear maps, $P_{\varepsilon}^{K}$ and $E_{\varepsilon}^{K}$ linking $\left[L^{2}(\Omega)^{N}\right]^{K^{N}}$ and $\mathbb{R}^{N n(\varepsilon)}$ such that $S_{\varepsilon}^{K}=E_{\varepsilon}^{K} S_{\varepsilon} P_{\varepsilon}^{K}$. To do so, we first introduce some notations connecting the two indices $p$ (indexing constant vectors in $\mathbb{R}^{N n(\varepsilon)}$ and $j$ (indexing vector functions in $\left.\left[L^{2}(\Omega)^{N}\right]^{K^{N}}\right)$.

Definition 3.1.1. Let $j=\left(j_{1}, \ldots, j_{N}\right)$ be the multi-integer which enumerates all the tubes in the periodic reference cell $K Y$. Each component of $j$ belongs to $\{0,1, \ldots, K-1\}$, and we use the notation $0 \leqq j \leqq K-1$ to indicate its range. Let $p=\left(p_{1}, \ldots, p_{N}\right\}$ be the multi-integer which enumerates all the tubes in $\Omega_{\varepsilon}$ (see (3)). We do not precisely describe the range of each of its components, and for simplicity the range of $p$ is denoted by $1 \leqq p \leqq n(\varepsilon)$. We define a third multi-integer $l=\left(l_{1}, \ldots, l_{N}\right)$ which enumerates all the periodic reference cells $\varepsilon(K Y)$ in $\Omega_{\varepsilon}$. For simplicity its range is denoted by $1 \leqq l \leqq n_{K}(\varepsilon)$. These three indexes are assumed to be related by the one-to-one map

$$
l_{m}=E\left(\frac{p_{m}}{K}\right), \quad j_{m}=p_{m}-K l_{m} \quad \forall m=1, \ldots, N
$$

where $E(\cdot)$ denotes the integer-part function.

The projection operator $P_{\varepsilon}^{K}$, which maps $\left[L^{2}(\Omega)^{N}\right]^{K^{N}}$ onto $\mathbb{R}^{N n(\varepsilon)}$, associates with any family of functions $\left(s_{j}(x)\right)_{0 \leq j \leq K-1}$ a collection of constant vectors $\left(s_{p}\right)_{0 \leqq p \leqq n(\varepsilon)}$, each of them obtained by simply taking the average of $s_{j}(x)$ on the cell $\varepsilon(K Y)_{l}$, where $p$ is related to $(l, j)$ through Definition 3.1.1. More precisely, we have

$$
\begin{aligned}
& P_{\varepsilon}^{K}:\left[L^{2}(\Omega)^{N}\right]^{K^{N}} \rightarrow \mathbb{R}^{N n(\varepsilon)}, \\
& \left(s_{j}(x)\right)_{0 \leqq j \leqq K-1} \rightarrow\left(s_{p}\right)_{1 \leqq p \leqq n(\varepsilon)}
\end{aligned}
$$

where

$$
s_{i}=\frac{1}{\left|\varepsilon(K Y)_{l}\right|} \int_{\varepsilon(K Y)_{l}} s_{j}(x) d x
$$

with $p$ related to $(l, j)$ by formula $(49)$.

On the other hand, the extension operator $E_{\varepsilon}^{K}$ is defined by

$$
\begin{gathered}
E_{\varepsilon}^{K}: \mathbb{R}^{N n(\varepsilon)} \rightarrow\left[L^{2}(\Omega)^{N}\right]^{K^{N}}, \\
\left(s_{p}\right)_{1 \leqq p \leqq n(\varepsilon)} \rightarrow\left(s_{j}(x)\right)_{0 \leqq j \leqq K-1}
\end{gathered}
$$

where

$$
\boldsymbol{s}_{j}(x)=\sum_{l} \chi_{\varepsilon}(K Y)_{l}(x) \boldsymbol{s}_{p}
$$


since $p$ is related to $(l, j)$ by formula (49), and where, by convention, $\boldsymbol{s}_{p}$ is taken equal to zero if, near the boundary of $\Omega$, some values of $l$ and $j$ yield an index $p$ which corresponds to a subcell containing no tube (or outside the domain $\Omega$ ).

Then, $S_{\varepsilon}^{K}$ is defined by the composition rule

$$
\begin{aligned}
S_{\varepsilon}^{K}:\left[L^{2}(\Omega)^{N}\right]^{K^{N}} & \rightarrow\left[L^{2}(\Omega)^{N}\right]^{K^{N},} \\
\left(s_{j}\right)_{0} \leqq j \leqq K-1 & \rightarrow E_{\varepsilon}^{K} S_{\varepsilon} P_{\varepsilon}^{K}\left(s_{j}\right)_{0 \leqq j \leqq K-1}
\end{aligned}
$$

where $S_{\varepsilon}$ is the original operator defined by (8) on $\mathbb{R}^{N n(\varepsilon)}$.

One can easily check that the adjoint operator of $P_{\varepsilon}^{K}$, denoted by $\left(P_{\varepsilon}^{K}\right)^{*}$, is nothing but $(\varepsilon K)^{-N} E_{\varepsilon}^{K}$, and that $P_{\varepsilon}^{K} E_{\varepsilon}^{K}$ is equal to the identity in $\mathbb{R}^{N n(\varepsilon)}$. Therefore, $S_{\varepsilon}^{K}$ is also self-adjoint and its spectrum is just the same as that of $S_{\varepsilon}$, except for the eigenvalue 0 , which is again an eigenvalue of infinite multiplicity. It is also worth noting that our old extended operator $\tilde{S_{\varepsilon}}$ is nothing but $S_{\varepsilon}^{K}$ with $K=1$.

The convergence analysis of this sequence of extensions $S_{\varepsilon}^{K}$ is amenable to the two-scale convergence method by appropriately choosing $\varepsilon K Y$ as the period of the oscillating test functions. It turns out that the corresponding limit operator $S^{K}$ has a quite complicated form since it mixes all the variables $s_{j}(x)$. However, $S^{K}$ can be diagonalized, and thus considerably simplified, by introducing the so-called Blochwave decomposition of the family $\left(s_{j}\right)_{0 \leq j \leq K-1}$. Our main results will be presented with this diagonal form of $S^{K}$ (each diagonal term being an operator from $L^{2}(\Omega)^{N}$ into itself). We call this blend of the two-scale convergence method and the Bloch-wave analysis a Bloch-wave homogenization procedure.

For the sake of completeness, we conclude this section by recalling the ad hoc discrete version of the Bloch-wave decomposition we shall use in the sequel (we call it discrete in contrast with the usual continuous Bloch-wave decomposition of functions in $L^{2}\left(\mathbb{R}^{N}\right)$; see $\left.[7,14,24,39]\right)$. Such a decomposition was already introduced for the same problem in a different context in [1]. With each family $\left(s_{j}\right)_{0 \leqq j \leqq K-1} \in\left(\mathbb{C}^{N}\right)^{K^{N}}$, we associate a $K Y$-periodic function, constant and equal to $\boldsymbol{s}_{j}$ in each subcell $Y_{j}$. In other words, denoting by $\chi_{Y_{j}}(y)$ the characteristic function of $Y_{j}$, we consider the function

$$
\boldsymbol{s}(y)=\sum_{j=0}^{K-1} \boldsymbol{s}_{j} \chi_{Y_{j}}(y) \quad \forall y \in K Y
$$

The Bloch-wave decomposition of $s(y)$ is given by

Lemma 3.1.2. There exists a unique family of constant vectors $\left(\boldsymbol{t}_{j}\right)_{0 \leqq j \leqq K-1}$ in $\mathbb{C}^{N}$ such that

$$
\boldsymbol{s}(y)=\sum_{j=0}^{K-1} \boldsymbol{t}_{j} e^{2 \pi i \theta_{j} \cdot E(y)} \quad \forall y \in K Y \quad \text { with } \theta_{j}=\frac{j}{K}
$$

where $E(\cdot)$ denotes the integer-part function. Moreover, Parseval's identity holds:

$$
\frac{1}{K^{N}} \int_{K Y}|\boldsymbol{s}(y)|^{2} d y=\frac{1}{K^{N}} \sum_{j=0}^{K-1}\left|\boldsymbol{s}_{j}\right|^{2}=\sum_{j=0}^{K-1}\left|\boldsymbol{t}_{j}\right|^{2} .
$$


Let $\mathscr{B}$ (for Bloch decomposition) denote the linear map which gives $K^{N / 2}\left(\boldsymbol{t}_{j}\right)$ in terms of $\left(s_{j}\right)$. The map $\mathscr{B}$ defines an isometry on $\left(\mathbb{C}^{N}\right)^{K^{N}}$. In particular, the adjoint $\mathscr{B} *$ of $\mathscr{B}$ is simply $\mathscr{B}^{-1}$.

Proof. For each $j=0, \ldots, K-1$, let us define $t_{j}$ by

$$
t_{j}=\frac{1}{K^{N}} \sum_{j=0}^{K-1} s_{j^{\prime}} e^{-2 \pi i j^{\prime} \cdot \theta_{j}}
$$

It suffices now to check that (55) holds with this definition of $t_{j}$ :

$$
\frac{1}{K^{N}} \sum_{j=0}^{K-1} \sum_{j^{\prime}=0}^{K-1} s_{j^{\prime}} e^{2 \pi i \theta_{j^{\prime}}\left(E(y)-j^{\prime}\right)}=\sum_{j^{\prime}=0}^{K-1} s_{j^{\prime}}\left(\frac{1}{K^{N}} \sum_{j=0}^{K-1} e^{2 \pi i \theta_{j^{\prime}}\left(E(y)-j^{\prime}\right)}\right) .
$$

If $E(y)=j^{\prime}$, the expression in parentheses on the right-hand side of (58) is equal to 1 . If $E(y) \neq j^{\prime}$, it is equal to 0 , thanks to a well-known property of the $K$-th roots of 1 in the complex plane (see [1] for details). Thus, this term is equal to $\chi_{y_{j^{\prime}}}(y)$, which proves (55). The proof of (56) is similar, so we omit it.

The net effect of the discrete Bloch-wave decomposition is to allow us to work with the family of frequencies $\left(t_{j}\right)_{0 \leqq j \leqq K-1}$ instead of the family of displacements $\left(\boldsymbol{s}_{j}\right)_{0 \leqq j \leqq K-1}$. In the next section, we shall see that the operator $T^{K}=\mathscr{B} S^{K} \mathscr{B} *$, defined on $\left[L^{2}(\Omega)^{N}\right]^{K^{N}}$, is diagonal, i.e., $T^{K}\left(t_{j}\right)=\left(T_{j}^{K} t_{j}\right)$, where each component $T_{j}^{K}$ is an operator on $L^{2}(\Omega)^{N}$. Thus, in the present context, it is easier to work with frequencies than displacements. Note that, as usual, the above Bloch-wave decomposition holds for families of complex-valued vectors. Even if the original family $\left(\boldsymbol{s}_{j}\right)$ is real, its image $\left(\boldsymbol{t}_{j}\right)$ under $\mathscr{B}$ is complex. Therefore, in the sequel we shall sometimes need to consider complex-valued functions. To simplify the exposition, we shall use the same notations for the usual Sobolev spaces considered as vector spaces of complex-valued, or real-valued, functions, according to the context. As usual, if $u$ is any complex number, then by $\bar{u}$ we mean the conjugate of $u$.

\subsection{Main results}

Since $S_{\varepsilon}^{K}$ has been introduced, we can now study its asymptotic behavior as $\varepsilon$ goes to zero. In Section 3.3 we shall prove the following generalization of Theorem 2.1.1.

Theorem 3.2.1. For each fixed $K \in \mathbb{N}^{*}$, the sequence $S_{\varepsilon}^{K}$ converges strongly to a limit $S^{K}$ in $\left[L^{2}(\Omega)^{N}\right]^{K^{N}}$, i.e., for any family $\left(\boldsymbol{s}_{j}(x)\right)_{0 \leqq j \leqq K-1}$,

$$
S_{s}^{K}\left(s_{j}\right) \rightarrow S^{K}\left(s_{j}\right) \text { in }\left[L^{2}(\Omega)^{N}\right]^{K^{N}} \text { strongly. }
$$

By using the Bloch-wave decomposition operator $\mathscr{B}$ defined in Lemma 3.1.2, the limit operator $S^{K}$ can be diagonalized, i.e.,

$$
S^{K}=\mathscr{B} * T^{K} \mathscr{B}, \quad \text { with } T^{K}=\operatorname{diag}\left[\left(T_{j}^{K}\right)_{0 \leqq j \leqq K-1}\right]
$$


where the entries $T_{j}^{K}$ are self-adjoint continuous operators in $L^{2}(\Omega)^{N}$, defined by

$$
T_{j}^{K} \boldsymbol{t}= \begin{cases}(A-I) \nabla u-(A-\theta I) \boldsymbol{t} & \text { if } j=0, \\ A^{j} \boldsymbol{t} & \text { if } j \neq 0,\end{cases}
$$

where $A$ is the usual homogenized matrix (defined by (15)), $\theta=\left|Y^{*}\right|$ the volume fraction of fluid, $u$ the unique solution of the usual homogenized problem

$$
\begin{aligned}
-\operatorname{div}(A \nabla u) & =\operatorname{div}((I-A) t) & & \text { in } \Omega, \\
u & =0 & & \text { on } \partial \Omega,
\end{aligned}
$$

and $A^{j}$ the Bloch-homogenized matrix with components $\left(A_{m m^{\prime}}^{j}\right)_{1 \leqq m, m^{\prime} \leqq N}$ defined by

$$
\bar{A}_{m m^{\prime}}^{j}=\int_{Y^{*}} \nabla w_{m}^{j}(y) \cdot \nabla \bar{w}_{m^{\prime}}^{j}(y) d y
$$

where $\left(w_{m}^{j}\right)_{1 \leqq m \leqq N}$ are solutions of the so-called cell problem at the Bloch frequency $\theta_{j}=(j / K)$ :

$$
\begin{array}{lr}
-\Delta w_{m}^{j}=0 & \text { in } Y^{*} \\
\left(\nabla w_{m}^{j}-e_{m}\right) \cdot n=0 & \text { on } \partial T \\
y \rightarrow e^{-2 \pi i \theta_{j} \cdot y} w_{m}^{j}(y) \text { is } Y^{*} \text {-periodic. } &
\end{array}
$$

Remark 3.2.2. Observe that the component $T_{0}^{K}$ of $T^{K}$ is nothing but the macroscopic limit operator $S$ defined in Section 2. The other components of $T^{K}$ are simple linear multiplication operators that represent the microscopic limit behavior of the sequence $S_{\varepsilon}^{K}$. Note also that the homogenized problem (62) is independent of $K$, so that the macroscopic fluid potential $u$ is also independent of $K$.

Remark 3.2.3. A function $w(y)$ satisfying the periodicity condition of the cell problem (64) is said to be $\left(e^{2 \pi i \theta_{j}}, Y^{*}\right)$-periodic; its properties have been extensively studied in $[1,12]$. We briefly recall their basic results. This class of functions can equivalently be characterized by the (generalized) periodicity condition

$$
w\left(y+j^{\prime}\right)=e^{2 \pi i j^{\prime} \cdot \theta_{j}} w(y) \quad \forall y \in Y^{*}, \quad \forall j^{\prime} \in \mathbb{Z}^{N} .
$$

The subspace of $H_{\text {loc }}^{1}\left(\mathbb{R}^{N}\right)$ consisting of such $\left(e^{2 \pi i \theta_{f}}, Y^{*}\right)$-periodic functions is a Hilbert space for the usual inner product in $H^{1}\left(Y^{*}\right)$ (see Theorem 2.1 of [1]). We denote it by $H_{\# \neq}^{1}\left(e^{2 \pi i \theta_{j}}, Y^{*}\right)$. Then, the cell problem $(64)$ is well-posed in this space $H_{\#}^{1}\left(e^{2 \pi i \theta_{j}}, Y^{*}\right)$ if $j \neq 0$, and in the quotient space $\left[H_{\#}^{1}\left(Y^{*}\right) / \mathbb{C}\right]$ if $j=0$ (see Lemma $3.1[1])$.

Let us now describe the spectrum $\sigma\left(S^{K}\right)$ of $S^{K}$. Since the Bloch-wave decomposition operator $\mathscr{B}$ is an isometry on $\mathbb{C}^{N}$, the spectrum of $S^{K}$ is exactly that of $T^{K}$, which is a diagonal operator whose diagonal entries $\left(T_{j}^{K}\right)_{0 \leqq j \leqq K-1}$ are linear, self-adjoint, continuous operators in $L^{2}(\Omega)^{N}$. Therefore its spectrum is nothing else 
than the union of the spectra of the operators $T_{j}^{K}$ for $j=0, \ldots, K-1$, i.e.,

$$
\sigma\left(S^{K}\right)=\sigma(S) \cup \bigcup_{j \neq 0} \sigma\left(T_{j}^{K}\right)
$$

Recall that $T_{0}^{K}$ is equal to $S$, which has a purely essential spectrum (see Theorems 2.1.4 and 2.1.5). If $j \neq 0, T_{j}^{K}$ is simply the multiplication operator by the Blochhomogenized matrix $A^{j}$ defined by (63). The description of $\sigma\left(T_{j}^{K}\right)$ follows easily from the definition (63) of $A^{j}$.

Proposition 3.2.4. For each fixed $j \neq 0,0 \leqq j \leqq K-1$, the Bloch-homogenized matrix $A^{j}$ is Hermitian and positive definite. Thus, the operator $T_{j}^{K}$ defined on $L^{2}(\Omega)^{N}$ by (61) is self-adjoint and nonnegative, and its spectrum coincides with the eigenvalues of $A^{j}$. All the elements of $\sigma\left(T_{j}^{K}\right)$ are eigenvalues of infinite multiplicity, and $T_{j}^{K}$ is therefore non-compact.

Collecting the above results concerning the spectra of the components $T_{j}^{K}$, we deduce that $S^{K}$ is a non-compact, self-adjoint operator whose spectrum is purely essential. Since $T^{\boldsymbol{K}}$ is diagonal, it is very easy to compute its spectral family in terms of the spectral families of its components. Indeed, if $\mathscr{F}_{j}^{K}(\lambda)$ denotes the spectral family of $T_{j}^{K}$ and $\mathscr{E}^{K}(\lambda)$ denotes that of $S^{K}$, then, for any $\left(s_{j}\right)_{0 \leqq j \leqq K-1} \in\left[L^{2}(\Omega)^{N}\right]^{K^{K}}$, $\mathscr{E}^{K}(\lambda)$ is simply given by

$$
\mathscr{E}^{K}(\lambda)\left(\boldsymbol{s}_{j}\right)=\mathscr{B}^{*} \operatorname{diag}\left[\mathscr{F}_{j^{\prime}}^{K}(\lambda)\right] \mathscr{B}\left(\boldsymbol{s}_{j}\right)
$$

Recall that for the isotropic case (i.e., $A=\alpha I$ ) we were able to obtain an explicit formula for $\mathscr{F}_{0}^{K}(\lambda)$ (see (17)). In order to describe $\mathscr{F}_{j}^{K}(\lambda)$ for $j \neq 0$, let us denote by $0<\lambda_{1}^{j} \leqq \cdots \leqq \lambda_{N}^{j}$ the eigenvalues, and by $\left(e_{m}^{j}\right)_{1 \leqq m \leqq N}$ the corresponding eigenvectors of the Bloch-homogenized matrix $A^{j}$. The spectral family $\mathscr{F}_{j}^{K}(\lambda)$ (for $j \neq 0$ ) is defined by

$$
\mathscr{F}_{j}^{K}(\lambda) t(x)=\sum_{\left\{m \mid \hat{\lambda}_{m}^{j} \leqq \lambda\right\}}\left(t(x) \cdot e_{m}^{j}\right) e_{m}^{j} .
$$

As in Section 2, we can deduce from the strong convergence of $S_{\varepsilon}^{K}$ to $S^{K}$ the convergence of the spectral family of $S_{\varepsilon}^{K}$ to that of $S^{K}$. We are again in a nonstandard situation where neither $S_{\varepsilon}^{K}$ nor its resolvent converges uniformly to their limits. Thus, we cannot expect pointwise convergence of the eigenvalues and we must content ourselves with the following result, which is an immediate consequence of Rellich's Theorem.

Theorem 3.2.5. Let $S_{\varepsilon}^{K}$ and $S^{K}$ be the operators on $\left[L^{2}(\Omega)^{N}\right]^{K^{N}}$ defined by (54) and (60) respectively. For all $\lambda \in \mathbb{R}$ such that $\lambda$ is not an eigenvalue of $S^{K}$, the spectral family $\mathscr{E}_{\varepsilon}^{K}(\lambda)$ of $S_{\varepsilon}^{K}$ converges strongly to that of $S^{K}$ in the following sense: For each $\left(s_{j}\right)_{0} \leqq j \leqq K-1 \in\left[L^{2}(\Omega)^{N}\right]^{K^{N}}$,

$$
\mathscr{E}_{\varepsilon}^{K}(\lambda)\left(s_{j}\right) \rightarrow \mathscr{E}^{K}(\lambda)\left(s_{j}\right) \quad \text { in }\left[L^{2}(\Omega)^{N}\right]^{K^{N}} \text { strongly as } \varepsilon \rightarrow 0 \text {. }
$$


Since the main goal of this paper is to study the pointwise convergence of the spectrum $\sigma\left(S_{\varepsilon}\right)=\sigma\left(S_{\varepsilon}^{K}\right) \backslash\{0\}$, we can infer some partial results from the strong convergence of the sequence $S_{\varepsilon}^{K}$, as we did in Section 2. More precisely, we define the limit set $\sigma_{\infty}$ of $\sigma\left(S_{\varepsilon}\right)$ as the set of all cluster points of sequences $\lambda_{\varepsilon}$ of eigenvalues of $S_{\varepsilon}$, i.e.,

$$
\sigma_{\infty}=\left\{\lambda \in \mathbb{R} \text { such that, up to a subsequence, } \exists \lambda_{\varepsilon} \in \sigma\left(S_{\varepsilon}\right), \lambda_{\varepsilon} \rightarrow \lambda\right\} .
$$

In other words, $\sigma_{\infty}$ is nothing but the $\Gamma$-limit of the sequence $\sigma\left(S_{\varepsilon}\right)$ in $\mathbb{R}$ (in the sense of the $\Gamma$-convergence of DE GroRgr [13]). Then, by application of Proposition 2.1.11 we have

$$
\sigma\left(S^{K}\right) \subset \sigma_{\infty}
$$

but the inclusion is generically strict. Note that, by virtue of (65), the spectrum of the macroscopic limit operator $S$ is included in the spectrum of the "macromicroscopic" limit operator $S^{K}$. Thus, the above results improve on those obtained in Section 2. Of course, we have the liberty of choosing any $K \in \mathbb{N}^{*}$, and by varying the cell size $K$ we obtain a wealth of information on $\sigma_{\infty}$. Indeed, as $K$ goes to infinity, the Bloch frequencies $\theta_{j}=(j / K)$ become dense in $] 0,1\left[^{N}\right.$. It is then natural to introduce a continuous vector parameter $\theta \in \mathbb{R}^{N}$, and to see if we can define a "Bloch-homogenized matrix" function $A(\theta)$ which coincides with the previous matrices $A^{j}$ for $\theta=\theta_{j}$, and whose entries $\left(A(\theta)_{m m^{\prime}}\right)_{1 \leqq m, m^{\prime} \leqq N}$ are defined by

$$
\overline{A_{m m^{\prime}}}(\theta)=\int_{Y^{*}} \nabla w_{m}^{\theta}(y) \cdot \nabla \bar{w}_{m^{\prime}}^{\theta}(y) d y
$$

where $\left(w_{m}^{\theta}\right)_{1 \leqq m \leqq N}$ are the unique solutions of the following cell problems at the Bloch frequency $\theta$ :

$$
\begin{array}{ll}
-\Delta w_{m}^{\theta}=0 & \text { on } Y^{*}, \\
\left(\nabla w_{m}^{\theta}-\boldsymbol{e}_{m}\right) \cdot \boldsymbol{n}=0 & \text { on } \partial T, \\
y \rightarrow e^{-2 \pi i \theta \cdot y} w_{m}^{\theta}(y) \text { is } Y^{*} \text {-periodic. } &
\end{array}
$$

As for the discrete case, any function satisfying the above periodicity condition is said to be $\left(e^{2 \pi i \theta}, Y^{*}\right)$-periodic, and the space of all functions of $H_{1 \mathrm{loc}}^{1}\left(\mathbb{R}^{N}\right)$ which are $\left(e^{2 \pi i \theta}, Y^{*}\right)$-periodic is a Hilbert space, denoted by $H_{* *}^{1}\left(e^{2 \pi i \theta}, Y^{*}\right)$ for the inner product in $H^{1}\left(Y^{*}\right)$ ) (see [1] for the details). Furthermore, problem (69) is wellposed in $H_{*}^{1}\left(e^{2 \pi i \theta}, Y^{*}\right)$ for $\theta \neq 0$, and in $\left[H_{\#}^{1}\left(Y^{*}\right) / \mathbb{C}\right]$ for $\theta=0$. In Section 3.4 we shall prove

Proposition 3.2.6. For any value of $\theta$, the Bloch-homogenized matrix $A(\theta)$ defined by (68) is Hermitian and positive-definite. Furthermore, as a function of $\theta$, it is a $[0,1]^{N}$ periodic, bounded function which is continuous in $] 0,1\left[{ }^{N}\right.$, but discontinuous at the origin $\theta=0$.

Since the main properties of the Bloch matrices have been established, we can now introduce a continuous family $T(\theta)$ of multiplication operators by these 
matrices acting on $L^{2}(\Omega)^{N}$. More precisely, for each $\theta$ in $] 0,1\left[^{N}\right.$, we define $T(\theta)$ by

$$
T(\theta) t=A(\theta) t \quad \forall t \in L^{2}(\Omega)^{N} .
$$

From Proposition 3.2.6 and the very definition of $T(\theta)$, it follows that $T(\theta)$ is self-adjoint and non-compact and that its spectrum coincides with the $N$ real positive eigenvalues of $A(\theta)$ that we denote by

$$
0<\lambda_{1}(\theta) \leqq \lambda_{2}(\theta) \leqq \cdots \leqq \lambda_{N}(\theta),
$$

where each eigenvalue is repeated as many times as its multiplicity. Since $A(\theta)$ is continuous in $\theta$, each eigenvalue $\lambda_{m}(\theta)$ is also a continuous function of $\theta$ on $] 0,1\left[^{N}\right.$ (see, e.g., [18], or, in the present situation, Chapter III in [11]).

Corollary 3.2.7. For each $m=1, \ldots, N$, the function $\lambda_{m}(\theta)$ is continuous on $] 0,1\left[^{N}\right.$, and bounded on $[0,1]^{N}$. Thus, the closure of the images of $] 0,1\left[^{N}\right.$ under the maps $\lambda_{m}(\cdot)$ are connected, closed bounded intervals in $] 0 ;+\infty[$ denoted by

$$
\left[a_{m}, b_{m}\right]=\overline{\lambda_{m}(] 0,1\left[{ }^{N}\right)}, \quad m=1, \ldots, N,
$$

where the bar denotes the closure in $\mathbb{R}$ and where

$$
a_{m}=\inf _{\theta \in] 0,1 \Gamma^{*}} \lambda_{m}(\theta), \quad b_{m}=\sup _{\theta \in] 0,1 \Gamma^{N}} \lambda_{m}(\theta)
$$

Remark 3.2.8. A detailed study of the behavior of $A(\theta)$ at the origin will be provided by Proposition 3.4.4. In particular, although $A(\theta)$ is not continuous at zero, we shall prove that all the eigenvalues of $A(0)$ are actually included in the so-called Bloch spectrum $\bigcup_{m=1}^{N}\left[a_{m}, b_{m}\right]$ (see Remark 3.4.5). For the moment, let us simply point out that, for the Bloch frequency $\theta=0$, the cell problem involves the usual periodicity condition, and problems (69) and (16) coincide. Comparing the definitions (15) of $A$ and (68) of $A(\theta)$, we easily check that $A(0)=\left|Y^{*}\right| I-A$. Thus, all the eigenvalues of $A(0)$ are also eigenvalues of $S$, but some of $S$ are not in $\sigma(A(0))$. For example, in the isotropic case $A=\alpha I$ (see Lemma 2.1.3), $S$ has two eigenvalues: $\left|Y^{*}\right|-\alpha$, which is also the unique eigenvalue of $A(0)$, and $\left|Y^{*}\right|+(1-2 \alpha) / \alpha$ which is specific to $S$. We remark in passing that, if the cubic symmetry of $Y *$ implies that $A(0)$ and $A$ are multiples of the identity, then in general this is not true any longer for $A(\theta)$ with $\theta \neq 0$.

It is now clear by the very construction of the operator $T(\theta)$ that, for all $K \geqq 1$ and for all $j \neq 0,0 \leqq j \leqq K-1$, the spectrum $\sigma\left(T_{j}^{K}\right)$ of the $j$-th component of $T^{\bar{K}}$ is contained in the union of all the intervals $\left[a_{m}, b_{m}\right]$, and by continuity, the closure of the union of all the spectra of the limit operators $T_{j}^{K}$, as $K$ goes to infinity, is nothing else than the Bloch spectrum $\bigcup_{m=1}^{N}\left[a_{m}, b_{m}\right]$. Of course, since the $\Gamma$-limit sets are closed, we deduce from (67) that

$$
\lim _{K \rightarrow+\infty} \sigma\left(S^{K}\right)=\left(\sigma(S) \cup \bigcup_{m=1}^{N}\left[a_{m}, b_{m}\right]\right) \subset \sigma_{\infty} .
$$


The question is now to see whether equality in (70) is achieved, which would imply that our limiting procedure recovers the whole limit spectrum in the limit when $K$ goes to infinity. In other words, we seek a characterization of $\sigma_{\infty}$, i.e., a so-called completeness result. However, there is another potential source of limit spectrum which is not taken into account by our analysis, namely, that of eigenvectors oscillating on the $\varepsilon$-scale while concentrating near the boundary $\partial \Omega$. The reason for expecting such a boundary-layer spectrum is the following. We know from Remarks 2.1.12 and 3.2.13 that the Bloch spectrum can also be obtained by rescaling the original $\varepsilon$-network of tubes to size 1 . Then, the boundary goes to infinity, and the limit domain is an infinite periodic arrangement of unit tubes (see [12]). The point is that it is also possible to center this rescaling procedure on some part of the boundary (rather than strictly inside the domain). In this case the limit domain is easily seen to be a half space filled with a periodic arrangement of tubes. It turns out that there actually exist sequences of eigenvectors concentrating on $\partial \Omega$. To account for this situation, we introduce $\sigma_{\text {boundary, }}$, the subset of $\sigma_{\infty}$ corresponding to this boundary-layer spectrum, defined by

$$
\begin{aligned}
& \sigma_{\text {boundary }}=\left\{\lambda \in \mathbb{R} / \exists\left(\lambda_{\varepsilon}, s_{\varepsilon}\right) \text { such that } \tilde{S}_{\varepsilon} s_{\varepsilon}=\lambda_{\varepsilon} s_{\varepsilon}, \lambda_{\varepsilon} \rightarrow \lambda,\right. \\
& \left.\qquad\left\|s_{\varepsilon}\right\|_{L^{2}(\Omega)}=1, \forall \omega \text { with } \bar{\omega} \subset \Omega,\left\|s_{\varepsilon}\right\|_{L^{2}(\omega)} \rightarrow 0\right\} .
\end{aligned}
$$

The main result of this paper states that, apart from this boundary-layer spectrum, the Bloch-wave homogenization method recovers all the limit spectrum.

Theorem 3.2.9. The limit set of the spectrum of the operator $S_{\varepsilon}$ is precisely made of three parts: the homogenized, the Bloch, and the boundary-layer spectrum, i.e.,

$$
\lim _{\varepsilon \rightarrow 0} \sigma\left(S_{\varepsilon}\right)=\sigma_{\infty}=\sigma(S) \cup \bigcup_{m=1}^{N}\left[a_{m}, b_{m}\right] \cup \sigma_{\text {boundary }}
$$

The proof of Theorem 3.2.9 is quite delicate and is the focus of Section 3.4. It involves two key ingredients. First, a careful analysis of the partial continuity of the matrix $A(\theta)$ near 0 is made (this is crucial for studying the asymptotic behavior of eigenvectors which oscillate on an intermediate length scale between $\varepsilon$ and 1; see Proposition 3.4.4). Second, for any sequence of eigenvectors, we introduce two successive measures which quantify its amplitude and direction of oscillations. The first so-called Bloch measure selects only those oscillations having a length scale of order $\varepsilon$, and decomposes them into Bloch frequencies. It can be seen as an ad hoc version of the well-known Wigner, or semi-classical, measure (see [16] and [20]). The second so-called rescaled Bloch measure keeps track of all oscillations having a length scale much larger than $\varepsilon$, but still smaller than 1 , and sorts them out according to their individual directions. This last type of measure is very similar, although specific to our context, to the recently introduced $H$-measures of P. Gérard [15] and L. Tartar [37].

Remark 3.2.10. Theorem 3.2.9 is optimal in the sense that, in general, the boundary-layer spectrum $\sigma_{\text {boundary }}$ is neither empty, nor included in $\sigma(S) \cup \bigcup_{m=1}^{N}\left[a_{m}, b_{m}\right]$. 


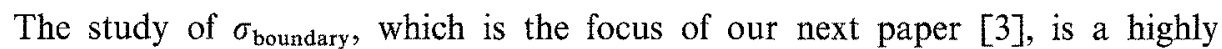
non-trivial problem. For a general domain $\Omega$ (with a smooth boundary not necessarily aligned with the periodic structure), we are unable to characterize $\sigma_{\text {boundary. }}$ We suspect its definition depends on the possible subsequence of periods $\varepsilon$ (a fact which is reminiscent of a recent work of F. SANTOSA \& M. VoGELIUS [34]). However, when the domain is exactly made of a finite number of entire periodic cells, we shall characterize $\sigma_{\text {boundary }}$ completely and prove that it is not included in the homogenized and Bloch parts of the limit spectrum (see [3]). By introducing a notion of two-scale convergence for boundary layers, we are able to adapt our Bloch-wave homogenization method in order to obtain a limit problem in a half space filled with a periodic arrangement of tubes.

Of course, if the domain $\Omega$ is a torus, there is no boundary, and thus no contribution of boundary layers in the limit spectrum! In this case, Theorem 3.2.9 can be further improved:

Corollary 3.2.11. Let $\Omega$ be a parallelepiped, $] 0, L_{1}[\times] 0, L_{2}[\times \cdots] 0, L_{N}[$, where the $\left(L_{m}\right)_{1 \leqq m \leqq N}$ are positive integers. Define the sequence of periods $\varepsilon_{n}=1 / n$. Assume that the unit tube in the periodic cell has cubic symmetry, or replace the Dirichlet boundary condition in the spectral problem (1) by a periodicity condition. Then, the limit spectrum reduces to

$$
\sigma_{\infty}=\sigma(S) \cup \bigcup_{m=1}^{N}\left[a_{m}, b_{m}\right]
$$

Remark 3.2.12. Theorem 3.2 .9 is a completeness result since it gives a decomposition of the limit spectrum into three parts: the homogenized or macroscopic spectrum $\sigma(S)$, the Bloch or microscopic spectrum $\bigcup_{m=1}^{N}\left[a_{m}, b_{m}\right]$, and the boundary-layer spectrum $\sigma_{\text {boundary }}$. From the point of view of "physical" intuition, this result is a little surprising because it excludes a "potential" part of the limit spectrum corresponding to mesoscopic oscillations (which are intermediate between microscopic and macroscopic oscillations). To explain this, note that $\sigma(S)$ corresponds to macroscopic eigenvectors, while the Bloch spectrum is obtained for $\varepsilon$-microscopic eigenvectors. One might wonder if some eigenvectors oscillating on an intermediate scale (such as $\sqrt{\varepsilon}$ ) would produce different limit eigenvalues. It turns out that this mesoscopic case is simply a limiting case of the Bloch-wave analysis when the number of subcells $K$ goes to infinity. In other words, the mesoscopic spectrum is already included in the Bloch spectrum. (The proof of this fact involves a careful analysis of the behavior of the matrix $A(\theta)$ near zero; see Proposition 3.4.4 and step 4 in the proof of Theorem 3.2.9.)

Remark 3.2.13. We feel that it is now appropriate to say a few words about the previous results obtained by C. CONCA \& M. VANNINATHAN in [12]. In Remark 2.1.12 we recalled that they studied the same problem as ours, but rescaled by a factor $\varepsilon^{-1}$ (this means that the tube size is fixed while the domain boundary goes to infinity, see (18)). By using a standard Bloch-decomposition method in an infinite 
domain, they only partially recover the limit spectrum. It turns out that they proved that

$$
\sigma_{\infty} \supset \bigcup_{m=1}^{N}\left[a_{m}, b_{m}\right]
$$

Our approach improves on theirs in two different respects: First, we give a more explicit characterization of the limit spectrum (see in particular Remark 3.4.5), and second we obtain the complete limit spectrum.

Remark 3.2.14. Theorem 3.2 .9 can be interpreted as a $\Gamma$-convergence type result for the spectrum of $S_{\varepsilon}$. Let us briefly explain why. For example, take the maximum eigenvalue $\lambda_{\varepsilon}\left(N n(\varepsilon)\right.$ ) of $S_{\varepsilon}$ (our argument works essentially in the same way for the minimum eigenvalue). It has the characterization

$$
\lambda_{\varepsilon}(N n(\varepsilon))=\max _{u \in H_{0}^{1}(\Omega)} \frac{\varepsilon^{-N} \sum_{p=1}^{n(\varepsilon)}\left(\int_{\Gamma_{p}^{z}} u n\right)^{2}}{\int_{\Omega_{\varepsilon}}|\nabla u|^{2}} .
$$

This maximization problem is easily seen to have at least one solution, since the embedding of $H_{0}^{1}(\Omega)$ in $L^{2}\left(\partial \Omega_{\varepsilon}\right)$ is compact, and any such solution satisfies the original spectral equation (7) with the maximal eigenvalue $\lambda_{\varepsilon}(N n(\varepsilon))$. As $\varepsilon$ goes to zero, one can study the $\Gamma$-convergence of the variational problem (72). By adapting the ideas of [8] and [21], we can prove that the $\Gamma$-limit of $(72)$ is simply

$$
\lambda_{\max }=\sup _{K \geqq 1} \sup _{\mathfrak{u} u_{1}} \frac{\int_{\Omega} \sum 0 \leqq j \leqq K-1\left(\int_{T_{j}}\left(\nabla_{x} u+\nabla_{y} u_{1}\right) d y\right)^{2} d x}{\int_{\Omega} \int_{K Y}\left|\nabla_{x} u+\nabla_{y} u_{1}\right|^{2} d x d y,}
$$

with

$$
u(x) \in H_{0}^{1}(\Omega), u_{1}(x, y) \in L^{2}\left(\Omega ; H_{\#}^{1}(K Y) / \mathbb{R}\right) .
$$

This type of result is classical for the homogenization of periodic non-convex energies: It implies that the usual "local cell problem" is not necessarily posed in a single period $Y$, but rather in the union of an (a priori unknown) number of periods $K Y$. Applying the Bloch-wave decomposition (see Lemma 3.1.2) in (73), we check that the value $\lambda_{\max }$ of the supremum coincides with the maximum value of $\lim _{K \rightarrow+\infty} \sigma\left(S^{K}\right)$. Therefore, this $\Gamma$-convergence method allows us to study the pointwise convergence of the minimum and maximum eigenvalues of $S_{\varepsilon}$. However, we do not know if it can succeed for the intermediate eigenvalues (in this case we have to rely on the min-max principle, whose asymptotic behavior is unclear). On the other hand, our Bloch-wave homogenization technique shows that any sequence of intermediate eigenvalues converges to a limit which belongs either to $\lim _{K \rightarrow+\infty} \sigma\left(S^{K}\right)$ or to the boundary-layer spectrum. In this sense, we can say that Theorem 3.2 .9 is a $\Gamma$-convergence-type result. On the link between $\Gamma$-convergence and Bloch waves, related results have been obtained in [17].

Remark 3.2.15. Let us discuss some concrete consequences of our main results. Theorem 3.2.9 shows that the limit of the spectrum of $S_{\varepsilon}$ always has a band structure. In the anisotropic case (i.e., when $Y^{*}$ does not have a cubic symmetry), there are, at least, two series of bands (which may well intersect): One is due to the 
Bloch-wave or microscopic part of the limit operator, the other arises from the macroscopic part $S$ (see Remark 2.1.6). In the isotropic case, the spectrum of $S$ is simply made of two points, and some simplifications arise in the number of independent "Bloch-wave" bands (see Remark 3.4.5). However, in all cases it is useless to compute numerically all the eigenvalues of $S_{\varepsilon}$ (for small $\varepsilon$ ), since in the limit $\sigma\left(S_{\varepsilon}\right)$ is dense in $\sigma_{\infty}$ ! Rather, it is preferable to compute the upper and lower bounds of these bands, which are amenable to simple computations in the unit cell $Y$.

From a physical point of view, one is usually interested in the lowest reasonance frequency $\omega_{\varepsilon}$ of the original vibration problem for a tubes bundle immersed in a fluid. Recall that we have rescaled the frequencies by

$$
\lambda_{\varepsilon}=\frac{k-m \omega_{\varepsilon}^{2}}{\varepsilon^{N} \rho \omega_{\varepsilon}^{2}} .
$$

Thus, low frequencies correspond to large $\lambda_{\varepsilon}$. An interesting open problem is to see if the maximal value of $\sigma_{\infty}$ is attained in $\sigma(S)$ (i.e., corresponds to a macroscopic displacement of the tubes) or in the Bloch-wave microscopic part. (Note that it is never attained in the boundary-layer spectrum by virtue of Remark 3.2.14). In the latter case, another problem is to find the values of the Bloch frequency $\theta$ for which the eigenvalue $\lambda_{N}(\theta)$ is maximal. The numerical computations in [1] suggest that, at least in the isotropic case in two dimensions, it is maximal when one of the components of $\theta$ is equal to 0 and the other to $\frac{1}{2}$.

\subsection{Convergence analysis}

This section is devoted to the proof of Theorem 3.2.1 on the strong convergence of the sequence $S_{\varepsilon}^{K}$. This proof is very similar to that of Theorem 2.1.1, although a little more tedious since the reference cell $K Y$ contains $K^{N}$ tubes instead of a single one. It relies on the homogenization of the problem

$$
\begin{aligned}
-\Delta u_{\varepsilon} & =0 & & \text { in } \Omega_{\varepsilon}, \\
\frac{\partial u_{\varepsilon}}{\partial n} & =P_{\varepsilon}^{K}\left(s_{j}\right) \cdot \boldsymbol{n} & & \text { on } \Gamma_{p}^{\varepsilon} \text { for } 1 \leqq p \leqq n(\varepsilon), \\
u_{\varepsilon} & =0 & & \text { on } \partial \Omega,
\end{aligned}
$$

where the family of displacements $\left(s_{j}\right)_{0 \leqq j \leqq K-1}$ belong to $\left[L^{2}(\Omega)\right]^{K^{N}}$. This problem has a unique solution $u_{\varepsilon} \in H^{1}\left(\Omega_{\varepsilon}\right)$, which, by virtue of Lemma 2.2 .3 , satisfies the a priori estimate

$$
\left\|u_{\varepsilon}\right\|_{H^{1}(\Omega)}^{2} \leqq C \sum_{j=0}^{K-1}\left\|s_{j}\right\|_{L^{2}(\Omega)^{N}}^{2}
$$

where the constant $C$ does not depend on $\varepsilon$. (As in Section 2, we use the same notation for a function in $H^{1}\left(\Omega_{\varepsilon}\right)$ and its bounded extension in $H^{1}(\Omega)$; see Lemma 2.2.2.)

To homogenize problem (74), we again use the two-scale convergence method and we obtain 
Proposition 3.3.1. The sequence $u_{\varepsilon}$ of solutions of $(74)$ converges weakly in $H_{0}^{1}(\Omega)$ to a limit $u$, and its gradient $\nabla u_{\varepsilon}$ two-scale converges to a limit $\nabla_{x} u(x)+\nabla_{y} u^{1}(x, y)$, where $\left(u, u^{1}\right)$ is the unique solution in $H_{0}^{1}(\Omega) \times L^{2}\left(\Omega ; H_{\#}^{1}\left(K Y^{*}\right) / \mathbb{R}\right)$ of the two-scale homogenized problem

$$
\begin{gathered}
-\operatorname{div}_{x}\left(\int_{K Y^{*}}\left(\nabla u(x)+\nabla_{y} u^{1}(x, y)\right) d y\right)=|T| \sum_{0 \leqq j \leqq K-1} \operatorname{div}_{x} s_{j} \quad \text { in } \Omega, \\
-\Delta_{y} u^{1}(x, y)=0 \quad \text { in } \Omega \times K Y^{*} \\
u(x)=0 \quad \text { on } \partial \Omega, \\
\left(\nabla u(x)+\nabla_{y} u^{1}(x, y)-s_{j}(x)\right) \cdot n=0 \quad \text { on } \Omega \times \partial T_{j}, \\
y \rightarrow u^{1}(x, y) \text { is } K Y^{*} \text {-periodic. }
\end{gathered}
$$

Furthermore,

$$
\lim _{\varepsilon \rightarrow 0} \int_{\Omega_{\varepsilon}}\left|\nabla u_{\varepsilon}\right|^{2} d x=\frac{1}{K^{N}} \int_{\Omega} \int_{K Y^{*}}\left|\nabla_{x} u(x)+\nabla_{y} u^{1}(x, y)\right|^{2} d x d y .
$$

The proof of Proposition 3.3.1 requires the following technical lemma.

Lemma 3.3.2. Let $\left(\boldsymbol{s}_{j}^{\varepsilon}\right)_{0 \leq j \leq K-1}$ be a family of bounded sequences in $L^{2}(\Omega)^{N}$ which converges weakly to a limit family $\left(s_{j}\right)_{0 \leqq j \leqq K-1}$. Then, the piecewise constant function $E_{\varepsilon} P_{\varepsilon}^{K}\left(s_{j}^{\varepsilon}\right) \in L^{2}(\Omega)^{N}$ defined by

$$
E_{\varepsilon} P_{\varepsilon}^{K}\left(\boldsymbol{s}_{j}^{\varepsilon}\right)(x)=\sum_{l, j}\left(\frac{1}{\left|\varepsilon(K Y)_{l}\right|} \int_{\varepsilon(K Y)_{l}} s_{j}^{\varepsilon}(x) d x\right) \chi_{Y_{i j}^{\varepsilon}}(x),
$$

where $\chi_{Y_{i}^{g}}(x)$ is the characteristic function of the jth subcell of the periodic cell $\varepsilon(K Y)_{l}$, two-scale converges to $s(x, y) \in L^{2}(\Omega \times K Y)^{N}$ defined by

$$
\boldsymbol{s}(x, y)=\sum_{j=0}^{K-1} \boldsymbol{s}_{j}(x) \chi_{Y_{j}}(y) .
$$

Moreover, if $s_{j}^{\varepsilon}$ converges strongly to $s_{j}$ in $L^{2}(\Omega)^{N}$ for all $j$, then

$$
\left\|P_{\varepsilon}^{K}\left(s_{j}^{\varepsilon}\right)(x)\right\|_{L^{2}(\Omega)^{N}}^{2} \rightarrow \frac{1}{K^{N}}\|s(x, y)\|_{L^{2}(\Omega \times K Y)^{N}}^{2} .
$$

Proof. Let $\varphi \in \mathscr{D}\left(\Omega ; C_{\#}^{\infty}(K Y)^{N}\right)$ be given. We check the definition of two-scale convergence:

$$
\begin{aligned}
\int_{\Omega} E_{\varepsilon} P_{\varepsilon}^{K}\left(s_{j}^{\varepsilon}\right)(x) \cdot \varphi\left(x, \frac{x}{\varepsilon}\right) d x & =\sum_{l, j}\left(\frac{1}{(\varepsilon K)^{N}} \int_{\varepsilon(K Y)_{l}} s_{j}^{\varepsilon}(x) d x\right) \cdot \int_{Y_{l_{j}^{\varepsilon}}^{\varepsilon}} \varphi\left(x, \frac{x}{\varepsilon}\right) d x \\
& =\frac{1}{K^{N}} \sum_{j=0}^{K-1} \int_{\Omega} s_{j}^{\varepsilon}(x) \cdot\left[\sum_{l}\left(\frac{1}{\varepsilon^{N}} \int_{Y_{Y_{j}}^{\varepsilon}} \varphi\left(x, \frac{x}{\varepsilon}\right) d x\right) \chi_{\varepsilon(K Y)_{l}}(x)\right] d x .
\end{aligned}
$$


It is easily seen that for each fixed $j$ the term between brackets converges strongly to $\int_{Y_{j}} \varphi(x, y) d y$. Thus we can pass to the limit and obtain

$$
\frac{1}{K^{N}} \sum_{j=0}^{K-1} \int_{\Omega} \mathbf{s}_{j}(x) \cdot \int_{Y_{j}} \varphi(x, y) d y
$$

which is the desired result. In the case where $\boldsymbol{s}_{j}^{\varepsilon}$ converges strongly to $\boldsymbol{s}_{j}$, (78) can be proved similarly by replacing in the above computations the test function $\varphi(x, x / \varepsilon)$ by $E_{\varepsilon} p_{\varepsilon}^{K}\left(s_{j}^{\varepsilon}\right)(x)$.

Proof of Proposition 3.3.1. By virtue of Proposition 2.2.1 there exists $\left(u, u^{1}\right)$ in $H_{0}^{1}(\Omega) \times L^{2}\left[\Omega ; H_{\# \#}^{1}(K Y)\right]$ such that (up to a subsequence) $u_{\varepsilon}$ and $\nabla u_{\varepsilon}$ two-scale converge to $u(x)$ and $\nabla u(x)+\nabla_{y} u^{1}(x, y)$ respectively. Next, we multiply equation (74) by $\phi(x)+\varepsilon \phi_{1}(x, x / \varepsilon)$ where $\phi \in \mathscr{D}(\Omega)$ and $\phi_{1} \in \mathscr{D}\left[\Omega ; C_{\#}^{\infty}(Y)\right]$. Integrating by parts in $\Omega_{\varepsilon}$, we get

$$
\begin{gathered}
\int_{\Omega} \chi\left(\frac{x}{\varepsilon}\right) \nabla u_{\varepsilon} \cdot\left(\nabla \phi(x)+\nabla_{y} \phi_{1}\left(x, \frac{x}{\varepsilon}\right)+\varepsilon \nabla_{x} \phi_{1}\left(x, \frac{x}{\varepsilon}\right)\right) d x \\
=\sum_{p=1}^{n(\varepsilon)}\left(P_{\varepsilon}^{K}\left(s_{j}\right)\right)_{p} \cdot\left(\int_{\Gamma_{p}^{\varepsilon}}\left(\phi(x)+\varepsilon \phi_{1}\left(x, \frac{x}{\varepsilon}\right)\right) n d s\right),
\end{gathered}
$$

where $\chi(y)$ is the characteristic function of $Y^{*}$. Applying Green's formula to the boundary integral on $\Gamma_{p}^{\varepsilon}$, we obtain (26) and, since $E_{\varepsilon} P_{\varepsilon}^{K}\left(s_{j}\right)$ is piecewise constant, we can rewrite the right-hand side of (79) as

$$
\int_{\Omega}\left(\chi\left(\frac{x}{\varepsilon}\right)-1\right) E_{\varepsilon} P_{\varepsilon}^{K}\left(s_{j}\right)(x) \cdot\left(\nabla_{x} \phi(x)+\nabla_{y} \phi_{1}\left(x, \frac{x}{\varepsilon}\right)+\varepsilon \nabla_{x} \phi_{1}\left(x, \frac{x}{\varepsilon}\right)\right) d x .
$$

By application of Lemma 3.3.2, $E_{\varepsilon} P_{\varepsilon}^{K}\left(s_{j}\right)$ two-scale converges, and passing to the limit in $(80)$ yields

$$
\frac{1}{K^{N}} \int_{\Omega} \int_{K Y}(\chi(y)-1) \sum_{0 \leqq j \leqq K-1} s_{j}(x) \chi_{Y_{j}}(y) \cdot\left(\nabla \phi(x)+\nabla_{y} \phi_{1}(x, y)\right) d x d y .
$$

On the other hand, the left-hand side of (79) passes easily to the limit using the definition of two-scale convergence. We obtain

$$
\begin{aligned}
& \frac{1}{K^{N}} \int_{\Omega} \int_{K Y^{*}}\left(\nabla u(x)+\nabla_{y} u^{1}(x, y)\right) \cdot\left(\nabla \phi(x)+\nabla_{y} \phi_{1}(x, y)\right) d x d y \\
& \quad=\frac{|T|}{K^{N}} \int_{\Omega} \phi(x) \sum_{j} \operatorname{div}_{x} s_{j}(x) d x+\frac{1}{K^{N}} \int_{\Omega}\left(\sum_{j} s_{j}(x) \cdot \int_{\Gamma_{j}} \phi_{1}(x, y) n_{y} d s\right) d x .
\end{aligned}
$$

We recognize in this identity the variational formulation for $\left(u, u^{1}\right)$ in the space $H_{0}^{1}(\Omega) \times L^{2}\left[\Omega ; H_{\#}^{1}\left(K Y^{*}\right)\right]$ of the two-scale homogenized problem (76). A standard 
application of the Lax-Milgram lemma shows existence and uniqueness of $\left(u, u^{1}\right)$, and hence the whole sequence $u_{\varepsilon}$ converges to its limit.

To obtain the convergence (77), we multiply equation (74) by $u_{\varepsilon}$ to obtain

$$
\int_{\Omega_{\varepsilon}}\left|\nabla u_{\varepsilon}\right|^{2} d x=\int_{\Omega}\left(\chi\left(\frac{x}{\varepsilon}\right)-1\right) E_{\varepsilon} P_{\varepsilon}^{K}\left(s_{j}\right)(x) \cdot \nabla u_{\varepsilon} d x .
$$

We can pass to the limit in the right-hand side of (82) since, by Lemma 3.3.2, $E_{\varepsilon} P_{\varepsilon}^{K}\left(s_{j}^{\varepsilon}\right)$ two-scale converges strongly (see Part 3 of Proposition 2.2.1). Thus

$$
\lim _{\varepsilon \rightarrow 0} \int_{\Omega_{\varepsilon}}\left|\nabla u_{\varepsilon}\right|^{2} d x=-\frac{1}{K^{N}} \int_{\Omega} \int_{K T} \sum_{j} s_{j}(x) \chi_{Y_{j}}(y) \cdot\left(\nabla u(x)+\nabla_{y} u^{1}(x, y)\right) d x d y .
$$

Finally, using the homogenized variational formulation (81) yields the desired result.

Proposition 3.3.1 yields implicitly the existence of a weak limit $S^{K}$ in $\left[L^{2}(\Omega)^{N}\right]^{K^{N}}$ of the sequence $S_{\varepsilon}^{K}$. Indeed, since $S_{\varepsilon}^{K}=E_{\varepsilon}^{K} S_{\varepsilon} P_{\varepsilon}^{K}$ and $\left(E_{\varepsilon}^{K}\right)^{*}=(\varepsilon K)^{N} P_{\varepsilon}^{K}$, we have

$$
\left\langle S_{\varepsilon}^{K}\left(s_{j}\right),\left(s_{j}\right)\right\rangle=K^{N} \sum_{p=1}^{n(\varepsilon)}\left(\int_{\Gamma_{p}^{e}} u_{\varepsilon} n d s\right) \cdot\left(P_{\varepsilon}^{K}\left(s_{j}\right)\right)_{p}
$$

where the brackets $\langle\cdot, \cdot\rangle$ indicate the standard inner product in $\left[L^{2}(\Omega)^{N}\right]^{K^{N}}$. Hence, from the energy identity (82), it follows that

$$
\left\langle S_{\varepsilon}^{K}\left(s_{j}\right),\left(s_{j}\right)\right\rangle=K^{N} \int_{\mathfrak{\Omega}_{s}}\left|\nabla u_{\varepsilon}\right|^{2} d x .
$$

Letting $\varepsilon$ go to zero in this identity, we see that Proposition 3.3.1 tells us that the limit of the right-hand side of (83) is a quadratic form in $\left(s_{j}\right)$. Therefore, $S_{\varepsilon}^{K}$ has a weak limit $S^{K}$ defined by

$$
\left\langle S^{K}\left(s_{j}\right),\left(s_{j}\right)\right\rangle=\iint_{\Omega} \int_{K Y^{*}}\left|\nabla_{x} u(x)+\nabla_{y} u^{1}(x, y)\right|^{2} d x d y,
$$

where $\left(u(x), u^{1}(x, y)\right)$ is the solution of (76) which depends linearly on $\left(s_{j}\right)$. Our next task is to show that the convergence of the sequence $S_{\varepsilon}^{K}$ is not merely weak but strong.

Proposition 3.3.3. The sequence $S_{\varepsilon}^{K}$ converges strongly in $\left[L^{2}(\Omega)^{N}\right]^{K^{N}}$ to its limit $S^{K}$ defined by (84).

Proof. Let $\left(r_{j}^{\varepsilon}\right)$ be any sequence of families of functions that converges weakly to a family $\left(r_{j}\right)$ in $\left[L^{2}(\Omega)^{N}\right]^{K^{N}}$. We have to prove that

$$
\lim _{\varepsilon \rightarrow 0}\left\langle S_{\varepsilon}^{K}\left(s_{j}\right),\left(r_{j}^{R}\right)\right\rangle=\left\langle S^{K}\left(s_{j}\right),\left(r_{j}\right)\right\rangle .
$$


To this end, we introduce a function $v_{\varepsilon}$ defined as the unique solution in $H^{1}\left(\Omega_{\varepsilon}\right)$ of problem (74) in which the right-hand side is precisely $P_{\varepsilon}^{K}\left(r_{j}\right)_{0 \leqq j \leqq K-1}$, i.e.,

$$
\begin{aligned}
-\Delta v_{\varepsilon} & =0 & & \text { in } \Omega_{\varepsilon}, \\
\frac{\partial v_{\varepsilon}}{\partial n} & =P_{\varepsilon}^{K}\left(\boldsymbol{r}_{j}^{\varepsilon}\right) \cdot \boldsymbol{n} & & \text { on } \Gamma_{p}^{\varepsilon} \text { for } 1 \leqq p \leqq n(\varepsilon), \\
v_{\varepsilon} & =0 & & \text { on } \partial \Omega .
\end{aligned}
$$

Multiplying the differential equation in (86) by $u_{\varepsilon}$ and integrating by parts in $\Omega_{\varepsilon}$, we easily obtain

$$
\left\langle S_{\varepsilon}^{K}\left(\boldsymbol{t}_{j}\right),\left(\boldsymbol{r}_{j}^{\varepsilon}\right)\right\rangle=K^{N} \int_{\Omega_{\varepsilon}} \nabla u_{\varepsilon} \cdot \nabla v_{\varepsilon} d x
$$

To prove the desired result, we have to pass to the limit in the right-hand side of (87). First, we need to homogenize problem (86). This is very similar to what we did in Proposition 3.3.1, except that here the right-hand side of (86) involves weakly converging sequences. For the sake of brevity, we simply sketch the main argument. Multiplying the differential equation in (86) by a test function $\phi(x)+\varepsilon \phi_{1}(x, x / \varepsilon)$, and integrating by parts yields

$$
\begin{aligned}
& \int_{\Omega} \chi\left(\frac{x}{\varepsilon}\right) \nabla v_{\varepsilon} \cdot\left(\nabla \phi(x)+\nabla_{y} \phi_{1}\left(x, \frac{x}{\varepsilon}\right)\right) d x \\
& \quad=\int_{\Omega}\left(\chi\left(\frac{x}{\varepsilon}\right)-1\right) E_{\varepsilon} P_{\varepsilon}^{K}\left(r_{j}^{\varepsilon}\right)(x) \cdot\left(\nabla_{x} \phi(x)+\nabla_{y} \phi_{1}\left(x, \frac{x}{\varepsilon}\right)\right) d x+o(1),
\end{aligned}
$$

where $o(1)$ is a term which goes to zero as $\varepsilon$ does. By application of Lemma 3.3.2, the piecewise constant function $E_{\varepsilon} P_{\varepsilon}^{K}\left(r_{j}^{\varepsilon}\right)(x)$ two-scale converges to $r(x, y) \in L^{2}(\Omega \times K Y)^{N}$ defined by

$$
r(x, y)=\sum_{j=0}^{k-1} r_{j}(x) \chi_{Y_{j}}(y)
$$

Thus, we can pass to the two-scale limit in (88), and it is easily seen that $v_{\varepsilon}$ and $\nabla v_{\varepsilon}$ two-scale converge towards $v(x)$ and $\nabla v(x)+\nabla_{y} v^{1}(x, y)$ respectively, where $\left(v, v^{1}\right)$ is the unique solution in $H_{0}^{1}(\Omega) \times L^{2}\left[\Omega ; H_{\mu}^{1}\left(K Y^{*}\right)\right]$ of the same two-scale homogenized system (76) where the right-hand sides $s_{j}$ are replaced by $\boldsymbol{r}_{j}$.

Now, to pass to the limit in (87), we use the strong two-scale convergence of $\nabla u_{\varepsilon}$ (see part 3 of Proposition 2.2.1), which is a consequence of the energy convergence (77). This yields

$$
\lim _{\varepsilon \rightarrow 0} \int_{\Omega_{z}} \nabla u_{\varepsilon} \cdot \nabla v_{\varepsilon} d x=\frac{1}{K^{N}} \int_{\Omega} \int_{K Y^{*}}\left(\nabla_{x} u+\nabla_{y} u^{1}\right) \cdot\left(\nabla_{x} v+\nabla_{y} v^{1}\right) d x d y
$$


An easy integration by parts in the two-scale homogenized system (76) combined with the definition (84) shows that

$$
\int_{\Omega} \int_{K Y^{*}}\left(\nabla_{x} u+\nabla_{y} u^{1}\right) \cdot\left(\nabla_{x} v+\nabla_{y} v^{1}\right) d x d y=\left\langle S^{K}\left(s_{j}\right),\left(r_{j}\right)\right\rangle,
$$

which is the desired result.

To complete the proof of Theorem 3.2.1, it remains to give a definition of the limit operator $S^{K}$ more explicit than (84), by using the Bloch-wave decomposition operator $\mathscr{B}$ introduced in Lemma 3.1.2. Since the solution $u^{1}(x, y)$ of the two-scale homogenized problem (76) is $K Y^{*}$-periodic, it is natural to decompose it into its Bloch components (as we did for the family $\left(s_{j}\right)$ ), and to try to reduce the homogenized problem (76) into $K^{N}$ sub-problems posed in $Y^{*}$. To this end, we recall the following lemma on the "Bloch-wave" orthogonal decomposition of $H_{\#}^{1}\left(K Y^{*}\right)$ (see Theorem 2.2 in [1]).

Lemma 3.3.4. For any multi-index $0 \leqq j \leqq K-1$, let $H_{\#}^{1}\left(e^{2 \pi i \theta_{j}}, Y^{*}\right)$ be the complex Hilbert subspace of $H_{*}^{1}\left(K Y^{*}\right)$ consisting of functions satisfying the so-called $\left(e^{2 \pi i \theta_{3}}, Y^{*}\right)$-periodicity condition:

$$
w\left(y+j^{\prime}\right)=e^{2 \pi i j^{\prime} \cdot \theta_{j}} w(y) \quad \forall y \in Y^{*}, \quad \forall j^{\prime} \in \mathbb{Z}^{N} \quad \text { with } \theta_{j}=\frac{j}{K} .
$$

They form an orthogonal decomposition of $H_{\#}^{1}\left(K Y^{*}\right)$ with respect to both inner products of $L^{2}\left(K Y^{*}\right)$ and $H^{1}\left(K Y^{*}\right)$, i.e.,

$$
H_{\#}^{1}\left(K Y^{*}\right)=\bigoplus_{0 \leqq j \leqq K-1} H_{\#}^{1}\left(e^{2 \pi i \theta_{j}}, Y^{*}\right) .
$$

Therefore, any function $w(y) \in H_{\#}^{1}\left(K Y^{*}\right)$ can be uniquely decomposed in

$$
w(y)=\sum_{j=0}^{K-1} w_{j}(y) e^{2 \pi i \theta_{j^{\prime}} y},
$$

with $w_{j}(y) \in H_{*}^{1}\left(Y^{*}\right)$ satisfying the Parseval identity

$$
\frac{1}{K^{N}} \int_{K Y^{*}}|w(y)|^{2} d y=\sum_{j=0}^{K-1} \int_{Y^{*}}\left|w_{j}(y)\right|^{2} d y .
$$

Proof of Theorem 3.2.1. By Lemma 3.3.4, the solution $u^{1}(x, y)$ of the two-scale homogenized problem (76) can be written as

$$
u^{1}(x, y)=\sum_{j=0}^{K-1} u_{j}(x, y) e^{2 \pi i \theta_{j} \cdot y}
$$

where each Bloch component $u_{j}(x, y)$ belongs to $L^{2}\left(\Omega ; H_{\#}^{1}\left(Y^{*}\right)\right)$. Furthermore, by Lemma 3.1.2, the right-hand side of (76) is

$$
s(y)=\sum_{j=0}^{K-1} s_{j} \chi_{Y_{j}}(y)=\sum_{j=0}^{K-1} t_{j} e^{2 \pi i \theta_{j} \cdot E(y)} .
$$


Substituting (90) and (91) into (76) and identifying coefficients corresponding to the same Bloch frequency $\theta_{j}$ (thanks to the orthogonality property of the Bloch-wave decomposition), we find that each $u_{j}$ is a solution of a boundary-value problem in $Y^{*}$ involving only $\boldsymbol{t}_{j}$.

If $j=0$, it is easily seen that $u_{0}(x, y)$ satisfies a system of equations which also involves $u(x)$. More precisely, $\left(u, u_{0}\right)$ is solution of the two-scale homogenized problem

$$
\begin{gathered}
-\operatorname{div}_{x}\left(\int_{Y^{*}}\left(\nabla u(x)+\nabla_{y} u_{0}(x, y)\right) d y\right)=|T| \operatorname{div}_{x} t_{0} \quad \text { in } \Omega, \\
-\Delta_{y} u_{0}(x, y)=0 \text { in } \Omega \times Y^{*}, \\
u(x)=0 \text { on } \partial \Omega, \\
\left(\nabla u(x)+\nabla_{y} u_{0}(x, y)-t_{0}(x)\right) \cdot n=0 \quad \text { on } \Omega \times \partial T, \\
y \rightarrow u^{0}(x, y) \text { is } Y^{*} \text {-periodic. }
\end{gathered}
$$

Observe that this problem is nothing but the usual two-scale homogenized problem obtained in Section 2.2 (see problem (30)). We recall that its solution $u_{0}(x, y)$ can be written in terms of $\nabla u(x), t_{0}(x)$ and the solutions $\left(w_{m}(y)\right)_{1 \leqq m \leqq N}$ of the usual cell problems (16) (see (31)). Eliminating the $y$ variable in (92) shows that $u$ is the unique solution of the homogenized problem (62).

On the other hand, if $j \neq 0$, then the average of $u_{j} e^{2 \pi i \theta_{j} \cdot y}$ on $K Y^{*}$ is zero, yielding a zero contribution of $u_{j}$ in the first equation of (76). Therefore, the subproblem for $u_{j}$ reduces to

$$
\begin{array}{ll}
\Delta_{y}\left(u_{j}(x, y) e^{2 \pi i \theta_{j} \cdot y}\right)=0 & \text { in } Y^{*}, \\
\left(\nabla_{y}\left(u_{j}(x, y) e^{2 \pi i \theta_{j} \cdot y}\right)-\boldsymbol{t}_{j}(x)\right) \cdot \boldsymbol{n}=0 & \text { on } \partial T, \\
y \rightarrow u_{j}(x, y) \text { is } Y^{*} \text {-periodic. } &
\end{array}
$$

By linearity, the solution $u_{j}$ of (93) can be computed in terms of $t_{j}(x)$ and of the solutions $\left(w_{m}^{j}(y)\right)_{1 \leqq m \leqq N}$ of the cell problem (64) at the Bloch frequency $\theta_{j}$ :

$$
u_{j}(x, y)=\sum_{m=1}^{N}\left(\boldsymbol{t}_{j}(x) \cdot \boldsymbol{e}_{m}\right) w_{m}^{j}(y) e^{-2 \pi i \theta_{j} \cdot y} .
$$

This completes the characterization of the homogenized solution $\left(u, u^{1}\right)$.

We now use this information in order to diagonalize $S^{K}$. From Parseval identity (89), the characterization (84) of the limit operator $S^{R}$ becomes

$$
\begin{aligned}
\frac{1}{K^{N}}\left\langle S^{K}\left(s_{j}\right)\left(s_{j}\right)\right\rangle= & \int_{\Omega} \int_{Y^{*}}\left|\nabla_{x} u(x)+\nabla_{y} u_{0}(x, y)\right|^{2} d x d y \\
& +\sum_{0 \leqq j \leqq K^{K}-1, j \neq 0} \int_{\Omega} \int_{Y^{*}}\left|\nabla_{y} u_{j}(x, y)\right|^{2} d x d y,
\end{aligned}
$$


and we have

$$
\begin{array}{r}
\int_{\Omega} \int_{Y^{*}}\left|\nabla_{x} u(x)+\nabla_{y} u_{0}(x, y)\right|^{2} d x d y=\int_{\Omega} S t_{0}(x) \cdot t_{0}(x) d x, \\
\int_{\Omega} \int_{Y^{*}}\left|\nabla_{y} u_{j}(x, y)\right|^{2} d x d y=\int_{\Omega} A^{j} t_{j}(x) \cdot t_{j}(x) d x .
\end{array}
$$

Since $K^{N / 2}\left(t_{j}\right)=\mathscr{B}\left(s_{j}\right)$, it is easy to conclude the proof of Theorem 3.2 .1 by checking that $S^{K}=\mathscr{B} * T^{K} \mathscr{B}$, where $T^{K}$ is the diagonal operator defined by (61).

\subsection{Completeness of the limit spectrum}

This section is devoted to the proof of the main result of this paper, namely, Theorem 3.2.9, which states the completeness of our analysis of the limit spectrum $\sigma_{\infty}$. It also contains the proof of several auxiliary results that are required in the course of the proof of Theorem 3.2.9. In particular, we prove the continuity of the matrix $A(\theta)$ on $] 0 ; 1\left[{ }^{N}\right.$ (Proposition 3.2.6), and study in great detail its behavior near 0 (Proposition 3.4.4). Recall that the entries of $A(\theta)$ are defined by

$$
\overline{A_{m m^{\prime}}}(\theta)=\int_{Y^{*}} \nabla w_{m}^{\theta}(y) \cdot \nabla \bar{w}_{m^{\prime}}^{\theta}(y) d y, \quad 1 \leqq m, m^{\prime} \leqq N,
$$

where $\left(w_{m}^{\theta}\right)_{1 \leqq m \leqq N}$ are the unique solutions of the cell problems at the Bloch frequency $\theta$ :

$$
\begin{array}{ll}
-\Delta w_{m}^{\theta}=0 & \text { in } Y^{*}, \\
\left(\nabla w_{m}^{\theta}-\boldsymbol{e}_{m}\right) \cdot \boldsymbol{n}=0 & \text { on } \partial T, \\
y \rightarrow e^{-2 \pi i \theta \cdot y} w_{m}^{\theta}(y) \text { is } Y^{*} \text {-periodic. } &
\end{array}
$$

Problem (97) admits the following variational formulation in the complex Hilbert space $H_{: 3}^{1}\left(e^{2 \pi i \theta}, Y^{*}\right)$ of $H_{\text {loc }}^{1}\left(\mathbb{R}^{N}\right)$ functions which satisfy the above so-called $\left(e^{2 \pi i \theta}, Y^{*}\right)$-periodicity condition:

$$
\int_{Y^{*}} \nabla w_{m}^{\theta}(y) \cdot \nabla \bar{\phi}^{\theta}(y) d y=\int_{\partial T} \boldsymbol{e}_{m} \cdot \boldsymbol{n} \bar{\phi}^{\theta}(s) d s \quad \forall \phi^{\theta} \in H_{\#}^{1}\left(e^{2 \pi i \theta}, Y^{*}\right)
$$

Remark 3.4.1. It is easily seen that adding any integer to any component of the parameter $\theta$ does not change the $\left(e^{2 \pi i \theta}, Y^{*}\right)$-periodicity condition and thus the definition of the space $H_{\#}^{1}\left(e^{2 \pi i \theta}, Y^{*}\right)$. Consequently the matrix $A(\theta)$ is $[0 ; 1]^{N}$ periodic in $\theta$. With no loss of generality, we can shift the domain of definition of $A(\theta)$ to the interval $\left[-\frac{1}{2}, \frac{1}{2}\right]^{N}$. This has the advantage that now there is only one point of discontinuity of $A(\theta)$, which is 0 , inside its domain of definition.

We begin by proving a Poincaré inequality in $H_{\#}^{1}\left(e^{2 \pi i \theta}, Y^{*}\right)$. 
Lemma 3.4.2. There exists a positive constant $C$ such that

$$
\left\|\phi^{\theta}\right\|_{L^{z}\left(Y^{*}\right)} \leqq \frac{C}{|\theta|}\left\|\nabla \phi^{\theta}\right\|_{L^{2}\left(Y^{*}\right)^{*}}
$$

for any non-zero $\theta \in\left[-\frac{1}{2} ; \frac{1}{2}\right]^{N}$, and for any $\phi^{\theta} \in H_{\#}^{1}\left(e^{2 \pi i \theta}, Y^{*}\right)$.

Proof. With no loss of generality we assume that the first component $\theta_{1}$ of $\theta$ is the largest one in absolute value. It is non-zero since $\theta$ is different from 0 . Let us prove the Poincare inequality for a function $\phi$ belonging to $H_{\#}^{1}\left(e^{2 \pi i \theta}, Y\right)$. For any point $y \in Y$,

$$
\phi\left(y+e_{1}\right)-\phi(y)=e^{2 \pi i \theta_{1}} \phi(y)-\phi(y)=\int_{0}^{1} \frac{\partial \phi}{\partial y_{1}}\left(y+t e_{1}\right) d t
$$

By the Schwarz inequality and integration over the cell $Y$, one obtains

$$
\|\phi\|_{L^{2}(Y)} \leqq \frac{1}{\left|e^{2 \pi i \theta_{1}}-1\right|}\left\|\nabla \phi^{\theta}\right\|_{L^{2}(Y)^{N}}
$$

It is easily seen that the constant in this equation is bounded by $C /|\theta|$. To obtain the same result in $Y^{*}$, one needs to introduce some extension operator from $H_{\#}^{1}\left(e^{2 \pi i \theta}, Y^{*}\right)$ into $H_{\#}^{1}\left(e^{2 \pi i \theta}, Y\right)$. It turns out that the usual extension operator from $H_{*}^{1}\left(Y^{*}\right)$ into $H_{\#}^{1}(Y)$ (introduced in [9]) also works in the present context. We leave the details to the reader.

To study the continuity of the matrix $A(\theta)$, we need a result of [1] which, roughly speaking, means that the space $H_{\#}^{1}\left(e^{2 \pi i \theta}, Y^{*}\right)$ is continuous in $\theta$. For the sake of completeness, we briefly sketch its proof.

Lemma 3.4.3. Let $\theta_{n}$ be a sequence converging to a (possibly zero) limit $\theta$ in $\left[-\frac{1}{2}: \frac{1}{2}\right]^{N}$. Then, for any $\phi^{\theta} \in H_{\#}^{1}\left(e^{2 \pi i \theta}, Y^{*}\right)$, there exists a sequence $\phi^{\theta_{n}} \in H_{\#}^{1}\left(e^{2 \pi i \theta_{n}}, Y^{*}\right)$ such that $\phi^{\theta_{n}}$ converges strongly to $\phi^{\theta}$ in $H^{1}\left(Y^{*}\right)$. Conversely, if $\phi^{\theta_{n}} \in H_{\#}^{1}\left(e^{2 \pi i \theta_{n}}, Y^{*}\right)$ is a sequence which converges strongly to $\phi^{\theta}$ in $H^{1}\left(Y^{*}\right)$, then this limit $\phi^{\theta}$ belongs to $H_{\#}^{1}\left(\mathrm{e}^{2 \pi i \theta}, Y^{*}\right)$.

Proof. Any function $\phi^{\theta} \in H_{\#}^{1}\left(e^{2 \pi i \theta}, Y^{*}\right)$ can be written as

$$
\phi^{\theta}(y)=\phi(y) e^{2 \pi i \theta \cdot y}
$$

where $\phi(y)$ belongs to $H_{* *}^{1}\left(Y^{*}\right)$. We define the sequence $\phi^{\theta_{n}}$ by

$$
\phi^{\theta_{n}}(y)=\phi(y) e^{2 \pi i \theta_{n} \cdot y} .
$$

One can easily check that $\phi^{\theta_{n}}$ converges strongly to $\phi^{\theta}$ in $H^{1}\left(Y^{*}\right)$. Conversely, if a sequence $\phi^{\theta_{n}}$, defined by

$$
\phi^{\theta_{n}}(y)=\phi_{n}(y) e^{2 \pi i \theta_{n} \cdot y}
$$


converges strongly to $\phi^{\theta}$ in $H_{*}^{1}\left(Y^{*}\right)$, then the sequence $\phi_{n}$ also converges strongly to a limit $\phi$ in $H_{\# \neq}^{1}\left(Y^{*}\right)$. One can pass to the limit in (100) to obtain (99), which proves that $\phi^{\theta}$ belongs to $H_{\#}^{1}\left(e^{2 \pi i \theta}, Y^{*}\right)$.

We are now in a position to prove the continuity of the matrix $A(\theta)$ on $\left[-\frac{1}{2} ; \frac{1}{2}\right]^{N} \backslash\{0\}$.

Proof of Proposition 3.2.6. Let $\theta_{n}$ be a sequence converging to a non-zero limit $\theta$ in $\left[-\frac{1}{2} ; \frac{1}{2}\right]^{N}$. Let us prove that the sequence of solutions $w_{m}^{\theta_{n}}$ of the cell problem (97) converges to the solution $w_{m}^{\theta}$. By the very definition (96) of $A(\theta)$, this is enough to prove its continuity. The variational formulation (98) yields the energy estimate

$$
\left\|\nabla w_{m}^{\theta_{n}}\right\|_{L^{2}\left(Y^{*}\right)}^{2}=\int_{\partial T} \boldsymbol{e}_{m} \cdot \boldsymbol{n} \bar{w}_{m}^{\theta_{n}}
$$

which, by using a standard extension operator, implies that the sequence $\nabla w_{m}^{\theta_{n}}$ is bounded in $L^{2}\left(Y^{*}\right)^{N}$. Up to a subsequence, it converges weakly in $L^{2}\left(Y^{*}\right)^{N}$, but the convergence is indeed strong by using (101) again.

Since $\theta$ is not $0,\left|\theta_{n}\right|$ is bounded away from 0 for sufficiently large $n$, and we can apply the Poincare inequality (Lemma 3.4.2) to a subsequence of $w_{m}^{\theta_{n}}$ which thus converges strongly in $H^{1}\left(Y^{*}\right)$. By Lemma 3.4.3 this limit belongs to $H_{*}^{1}\left(e^{2 \pi i \theta}, Y^{*}\right)$, and we can pass to the limit in the variational formulation for $w_{m}^{\theta_{n}}$. This proves that the limit is nothing but $w_{m}^{\theta}$, the solution of the cell problem (97).

Note that this argument for proving the continuity of $A(\theta)$ does not work at $\theta=0$. Indeed, in this case it may well happen that the $L^{2}\left(Y^{*}\right)$ norm of the sequence $w_{m}^{\theta_{n}}$ goes to infinity. It turns out that the limit of $w_{m}^{\theta_{n}}$ is not necessarily $w_{m}^{0}$ when $\theta_{n}$ goes to 0 , and thus $A(\theta)$ is not continuous at 0 . However, we now prove that $A\left(\theta_{n}\right)$ has a limit when $\theta_{n}$ goes to zero along rays of constant direction. More precisely, we have

Proposition 3.4.4. Let $\theta_{n}$ be a sequence converging to 0 , and such that $\theta_{n} /\left|\theta_{n}\right|$ converges to a unit vector $\xi$. Then, the matrix $A\left(\theta_{n}\right)$ converges to a real, positivedefinite matrix $\hat{A}(\xi)$ which is defined by its entries

$$
\hat{A}_{m m^{\prime}}(\xi)=\int_{Y^{*}} \nabla v_{m}^{\xi}(y) \cdot \nabla v_{m^{\prime}}^{\xi}(y) d y, \quad 1 \leqq m, m^{\prime} \leqq N,
$$

where $\left(v_{m}^{\xi}\right)_{1 \leqq m \leqq N}$ are the unique solutions in $H_{\#}^{1}\left(Y^{*}\right) / \mathbb{R}$ of the problems

$$
\begin{array}{ll}
-\Delta v_{m}^{\xi}=0 & \text { in } Y^{*}, \\
\frac{\partial v_{m}^{\xi}}{\partial n}=\boldsymbol{e}_{m} \cdot \boldsymbol{n}+\left(\xi \cdot \int_{Y^{*}} \nabla v_{m}^{\xi}+|T| \xi \cdot \boldsymbol{e}_{m}\right) \xi \cdot \boldsymbol{n} & \text { on } \partial T, \\
y \rightarrow v_{m}^{\xi}(y) \text { is } Y^{*} \text {-periodic. } &
\end{array}
$$


Furthermore, this matrix $A(\xi)$ is related to $A(0)$ by

$$
\hat{A}(\xi)=A(0)+(1-A(0) \xi \cdot \xi)^{-1}(A(0) \xi+|T| \xi) \otimes(A(0) \xi+|T| \xi),
$$

which proves that $\hat{A}(\xi)$ is a continuous function of $\xi$ and that $A(\theta)$ is not continuous at $\theta=0$.

Proof. Let $w_{m}^{\theta_{n}}$ be the solution of (97) (to simplify the notation, we drop the index $m$ in the sequel). We rewrite $w^{\theta_{n}}$ as

$$
w^{\theta_{n}}(y)=\left(w_{n}(y)+m_{n}\right) e^{2 \pi i \theta_{n} \cdot y}
$$

where $w_{n}$ belongs to $H_{\#}^{1}\left(Y^{*}\right)$ and has zero average, and where $m_{n}$ is a complex number. We choose a sequence of test function $\phi^{\theta_{n}} \in H_{\#}^{1}\left(e^{2 \pi i \theta_{n}}, Y^{*}\right)$ defined by

$$
\phi^{\theta_{n}}(y)=\left(\phi(y)+\mu_{n}\right) e^{2 \pi i \theta_{n} \cdot y}
$$

where $\phi$ belongs to $H_{\#}^{1}\left(Y^{*}\right)$ and has zero average, and $\mu_{n}$ is a complex number which goes as $\mu /\left|\theta_{n}\right|$ when $n$ goes to infinity (with $\mu \in \mathbb{C}$ ). Plugging these expressions into the variational formulation (98), we obtain

$$
\int_{Y^{*}}\left(\nabla w_{n}+2 \pi i \theta_{n}\left(w_{n}+m_{n}\right)\right) \cdot\left(\nabla \bar{\phi}-2 \pi i \theta_{n}\left(\bar{\phi}+\bar{\mu}_{n}\right)\right)=\int_{\partial T} \boldsymbol{e} \cdot \boldsymbol{n}\left(\bar{\phi}+\bar{\mu}_{n}\right) e^{-2 \pi i \theta_{n} \cdot y}
$$

Since $w_{n}$ has mean value zero in $Y^{*}$, the Poincare-Wirtinger inequality yields the a priori estimate

$$
\left\|w_{n}\right\|_{H^{1}\left(Y^{*}\right)} \leqq C
$$

while Lemma 3.4 .2 gives

$$
\left|\theta_{n} m_{n}\right| \leqq C .
$$

Consequently, up to a subsequence, $w_{n}$ converges weakly to $w$ in $H_{\#}^{1}\left(Y^{*}\right)$ and $\theta_{n} m_{n}$ converges to $\xi m$ for some $\xi \in \mathbb{C}$. Thus, we can pass to the limit in (105):

$$
\int_{Y^{*}} \nabla w \cdot \nabla \bar{\phi}+4 \pi^{2} m \bar{\mu}+2 \pi i m \xi \cdot \int_{Y^{*}} \nabla \bar{\phi}-2 \pi i \bar{\mu} \xi \cdot \int_{Y^{*}} \nabla w=-\int_{T} \boldsymbol{e} \cdot(\nabla \bar{\phi}-2 \pi i \bar{\mu} \xi) .
$$

This holds for any $\phi \in H_{*}^{1}\left(Y^{*}\right)$ with zero average and for any $\mu \in \mathbb{C}$. By varying $\mu$, we obtain

$$
m=\frac{2 \pi i \xi}{4 \pi^{2}} \cdot\left(|T| e+\int_{Y^{*}} \nabla w\right)
$$

which in turn yields

$$
\int_{Y^{*}} \nabla w \cdot \nabla \bar{\phi}-\left(\xi \cdot \int_{Y^{*}} \nabla w\right)\left(\xi \cdot \int_{Y^{*}} \nabla \bar{\phi}\right)=\int_{\partial T}(\boldsymbol{e}+|T|(\xi \cdot \boldsymbol{e}) \xi) \cdot \boldsymbol{n} \bar{\phi}
$$

for any $\phi \in H_{* *}^{1}\left(Y^{*}\right)$ with zero average. It is easy to check that the left-hand side of (106) is coercive on $H_{*}^{1}\left(Y^{*}\right) / \mathbb{C}$, uniformly in $\xi$, and thus that there exists a unique 
solution of $(106)$ in $H_{\sharp t}^{1}\left(Y^{*}\right) / \mathbb{C}$. By taking $\phi$ to be real, we check that the solution $w$ is purely real and that it coincides with $v^{\xi}$, the solution of (103). By linearity, $v^{\xi}$ can be computed in terms of $w^{0}(e)$ and $w^{0}(\xi)$, solutions of the cell problem (97) at the Bloch frequency 0 , and with right-hand side $e$ and $\xi$ respectively. A simple calculation yields

$$
\begin{aligned}
v^{\xi} & =w^{0}(\boldsymbol{e})+\frac{(A(0) \boldsymbol{e}+|T| \boldsymbol{e}) \cdot \xi}{1-A(0) \xi \cdot \xi} w^{0}(\xi), \\
\lim _{\theta_{n} \rightarrow 0, \theta_{n} / \theta_{n} \mid \rightarrow \xi} A\left(\theta_{n}\right) \boldsymbol{e} \cdot \boldsymbol{e} & =\hat{A}(\xi) \boldsymbol{e} \cdot \boldsymbol{e} \\
& =A(0) \boldsymbol{e} \cdot \boldsymbol{e}+\frac{((A(0) \boldsymbol{e}+|T| \boldsymbol{e}) \cdot \xi)^{2}}{1-A(0) \xi \cdot \xi},
\end{aligned}
$$

which is the desired result.

Remark 3.4.5. Proposition 3.4.4 tells us that $\hat{A}(\xi)$ differs from $A(0)$ by a positive rank-one matrix. Let us point out two consequences of this: First, the eigenvalues of $\hat{A}(\xi)$ are always greater than or equal to those of $A(0)$. Second, any eigenvalue $\lambda_{0}$ of $A(0)$ (with a corresponding eigenvector $\xi_{0}$ ) is also an eigenvalue of $\widehat{A}(\xi)$ for any choice of $\xi$ orthogonal to $\xi_{0}$. Thus, the eigenvalues of $A(0)$ are always included in the Bloch spectrum $\bigcup_{m=1}^{N}\left[a_{m}, b_{m}\right]$ (see (70)). In the isotropic case (i.e., $A(0)=a^{0} \mathrm{Id}$ ), for any value of $\xi, \hat{A}(\xi)$ has $N-1$ eigenvalues equal to $a^{0}$ and one equal to $a^{0}+\left(a^{0}+|T|\right)^{2} /\left(1-a^{0}\right)$. In particular, this proves that even if $\hat{A}(\xi)$ is not a constant function of $\xi$, its eigenvalues do not depend on $\xi$. It also proves that the a priori $N$ distinct bands in the Bloch-wave spectrum collapse in at most two distinct bands since $N-1$ of them intersect.

We now turn to the proof of Theorem 3.2.9 on the completeness of the limit spectrum $\sigma_{\infty}$. As in Section 2, we consider the extended operator $\tilde{S}_{\varepsilon}$ acting on $L^{2}(\Omega)^{N}$. Let $\lambda$ be any value in the limit set $\sigma_{\infty}$ of the spectra $\sigma\left(\tilde{S}_{\varepsilon}\right)$. By definition, there exists a subsequence (still denoted by $\varepsilon$ ) of eigenvalues $\lambda_{\varepsilon}$ and eigenvectors $\boldsymbol{s}_{\varepsilon}$ such that

$$
\begin{aligned}
\tilde{S}_{\varepsilon} \boldsymbol{s}_{\varepsilon} & =\lambda_{\varepsilon} \boldsymbol{s}_{\varepsilon} \quad \text { with }\left\|\boldsymbol{s}_{\varepsilon}\right\|_{L^{2}(\Omega)^{x}}=1, \\
\lambda_{\varepsilon} & \rightarrow \lambda .
\end{aligned}
$$

Our goal is to prove that $\lambda$ does indeed belong to $\sigma(S) \cup\left(\bigcup_{m=1}^{N}\left[a_{m}, b_{m}\right]\right) \cup \sigma_{\text {boundary }}$. We recall that $\widetilde{S}_{\varepsilon} s_{\varepsilon}$ is defined by

$$
\widetilde{S}_{\varepsilon} \boldsymbol{s}_{\varepsilon}=\sum_{p=1}^{n(\varepsilon)} \varepsilon^{-N}\left(\int_{\Gamma_{p}^{\varepsilon}} u_{\varepsilon} n\right) \chi_{Y_{p}^{\varepsilon}}(x)
$$

where $u_{\varepsilon}$ is the solution of

$$
\begin{aligned}
-\Delta u_{\varepsilon} & =0 & & \text { in } \Omega_{\varepsilon}, \\
\frac{\partial u_{\varepsilon}}{\partial n} & =\varepsilon^{-N}\left(\int_{Y_{p}^{\varepsilon}} s_{\varepsilon}(x) d x\right) \cdot \boldsymbol{n} & & \text { on } \Gamma_{p}^{\varepsilon} \text { for } 1 \leqq p \leqq n(\varepsilon), \\
u_{\varepsilon} & =0 & & \text { on } \partial \Omega .
\end{aligned}
$$


The following lemma establishes the connection between $\boldsymbol{s}_{\varepsilon}$ and $\mathfrak{u}_{\varepsilon}$.

Lemma 3.4.6. The solution $u_{\varepsilon}$ of (109) satisfies the estimates

$$
0<c \leqq\left\|u_{\bar{\alpha}}\right\|_{H^{1}(\Omega)} \leqq C,
$$

where $c$ and $C$ are two positive constants independent of $\varepsilon$. Furthermore, the sequence $\boldsymbol{s}_{\varepsilon}$ converges weakly to zero in $L^{2}(\Omega)^{N}$ if and only if $u_{\varepsilon}$ converges weakly to zero in $H_{0}^{1}(\Omega)$.

Proof. The estimates for $u_{\varepsilon}$ are deduced from Lemma 2.2.3 since $\left\|\boldsymbol{s}_{\varepsilon}\right\|_{L^{2}(\Omega)^{\mu}}=1$. The second statement is a direct consequence of the proof of Proposition 2.2.6 on the homogenization of problem (109).

Proof of Theorem 3.2.9. The proof is divided in four steps corresponding to four different behaviors of the sequence of eigenvectors $s_{\varepsilon}$ which cover all possible cases. Step 1: Macroscopic convergence of the eigenvectors. Since $s_{\varepsilon}$ is bounded in $L^{2}(\Omega)^{N}$, up to a subsequence, it converges weakly to a limit $s$. Assume that this limit is not zero, i.e., that the sequence $\boldsymbol{s}_{\varepsilon}$ has macroscopic oscillations. Then, multiplying (108) by a test function, one can pass to the limit thanks to the strong convergence of the operator $\widetilde{S}_{\varepsilon}$. Since $s \neq 0$, this shows that $s$ is an eigenvector associated with the eigenvalue $\lambda$ for the macroscopic limit operator $S$. This proves that $\lambda$ belongs to $\sigma(S)$.

Step 2: Concentration of the eigenvectors on the boundary. Assume that for any open subset $\omega$ such that $\bar{\omega} \subset \Omega$, the sequence $s_{\varepsilon}$ satisfies

$$
\lim _{\varepsilon \rightarrow 0}\left\|\boldsymbol{s}_{\varepsilon}\right\|_{L^{z}(\omega)}=0
$$

Then, by definition (71) of the boundary-layer spectrum, the corresponding sequence of eigenvalues $\lambda_{\varepsilon}$ converges to a limit $\lambda \in \sigma_{\text {boundary }}$.

Step 3: $\varepsilon$-Microscopic convergence of the eigenvectors. We now consider a sequence $s_{\varepsilon}$ which converges to 0 weakly in $L^{2}(\Omega)^{N}$ and such that there exists at least one open subset $\omega$, with $\bar{\omega} \subset \Omega$, and a strictly positive constant $c>0$ satisfying

$$
\lim _{\varepsilon \rightarrow 0}\left\|\boldsymbol{s}_{\varepsilon}\right\|_{L^{2}(\omega)} \geqq c>0 .
$$

Let $\phi \in \mathscr{D}(\Omega)$ be a smooth function with compact support in $\Omega$ and identically equal to 1 in $\omega$. The first key idea is to construct a "quasi-eigenvector", with fixed compact support in $\Omega$, by multiplying the true eigenvector $\boldsymbol{s}_{\varepsilon}$ by the cut-off function $\phi$. To simplify the sequel, it is better to have a piecewise constant quasi-eigenvector in each cell $Y_{p}^{\varepsilon}$. Therefore, we apply to $\phi s_{\varepsilon}$ the operator $E_{\varepsilon} P_{\varepsilon}$ which projects on such piecewise constant functions $\left(E_{\varepsilon}\right.$ and $P_{\varepsilon}$ are defined by (10) and (11)), and we normalize it. 
Lemma 3.4.7. The function $\boldsymbol{t}_{\varepsilon}$ defined by

$$
\boldsymbol{t}_{\varepsilon}=\frac{E_{\varepsilon} P_{\varepsilon}\left(\phi s_{\varepsilon}\right)}{\left\|E_{\varepsilon} P_{\varepsilon}\left(\phi s_{\varepsilon}\right)\right\|_{L^{2}(\Omega)^{N}}}
$$

is said to be a quasi-eigenvector since it satisfies

$$
\lim _{\varepsilon \rightarrow 0}\left\|\tilde{S}_{\varepsilon} \boldsymbol{t}_{\varepsilon}-\lambda_{\varepsilon} \boldsymbol{t}_{\varepsilon}\right\|_{L^{2}(\Omega)^{N}}=0
$$

Proof. Let us define $v_{\varepsilon}$ as the solution of

$$
\begin{aligned}
-\Delta v_{\varepsilon} & =0 & & \text { in } \Omega_{\varepsilon}, \\
\frac{\partial v_{\varepsilon}}{\partial n} & =\varepsilon^{-N}\left(\int_{\boldsymbol{Y}_{p}^{e}} \boldsymbol{t}_{\varepsilon}(x) d x\right) \cdot \boldsymbol{n} & & \text { on } \Gamma_{p}^{\varepsilon}, \text { for } 1 \leqq p \leqq n(\varepsilon), \\
v_{\varepsilon} & =0 & & \text { on } \partial \Omega .
\end{aligned}
$$

Let us first prove that $w_{\varepsilon}=v_{\varepsilon}-\phi u_{\varepsilon} /\left\|E_{\varepsilon} P_{\varepsilon}\left(\phi \mathbf{s}_{\varepsilon}\right)\right\|$ converges to 0 strongly in $H_{0}^{1}(\Omega)$. To simplify the notation, we denote by $a_{\varepsilon}$ the inverse of $\left\|E_{\varepsilon} P_{\varepsilon}\left(\phi s_{\varepsilon}\right)\right\|_{L^{2}(\Omega)^{*}}$, i.e.,

$$
w_{\varepsilon}(x)=v_{\varepsilon}(x)-a_{\varepsilon} \phi(x) u_{\varepsilon}(x) .
$$

We have

$$
\begin{aligned}
\int_{\Omega_{\varepsilon}}\left|\nabla w_{\varepsilon}\right|^{2} d x= & \int_{\Omega_{\varepsilon}} \nabla v_{\varepsilon} \cdot \nabla w_{\varepsilon} d x-a_{\varepsilon} \int_{\Omega_{\varepsilon}} \nabla u_{\varepsilon} \cdot \nabla\left(\phi w_{\varepsilon}\right) d x \\
& +a_{\varepsilon} \int_{\Omega_{\varepsilon}} \nabla \phi \cdot\left(w_{\varepsilon} \nabla u_{\varepsilon}-u_{\varepsilon} \nabla w_{\varepsilon}\right) d x .
\end{aligned}
$$

By assumption, $s_{\varepsilon}$ converges weakly to 0 in $L^{2}(\Omega)^{N}$, and the same is true for $t_{\varepsilon}$ by construction. Thus, by Lemma $3.4 .6, u_{\varepsilon}$ and $v_{\varepsilon}$ converge weakly to 0 in $H^{1}(\Omega)$, and strongly in $L^{2}(\Omega)$ by the Rellich theorem. Moreover, in view of (110), the scalar $a_{\varepsilon}$ is bounded. Therefore the last term in the right-hand side of (114) tends to 0 . On the other hand, by integration by parts and by using (109) and (113), the two first terms are equal to

$$
\sum_{p=1}^{n(\varepsilon)} \varepsilon^{-N}\left\{\left(\int_{Y_{p}^{\delta}} a_{\varepsilon} E_{\varepsilon} P_{\varepsilon}\left(\phi \boldsymbol{S}_{\varepsilon}\right) d x\right) \cdot\left(\int_{\Gamma_{p}^{\varepsilon}} w_{\varepsilon} n d s\right)-a_{\varepsilon}\left(\int_{Y_{p}^{\varepsilon}} \boldsymbol{s}_{\varepsilon} d x\right) \cdot\left(\int_{\Gamma_{p}^{\varepsilon}} \phi w_{\varepsilon} n d s\right)\right\} .
$$

Recalling that $s_{\varepsilon}$ is constant in each cell $Y_{p}^{\varepsilon}$, and replacing $\phi$ by its average value in each elementary integral, we find that the difference in this expression cancels out up to a remainder term which is easily seen to be bounded by

$$
\begin{aligned}
\varepsilon a_{\varepsilon}\|\phi\|_{C^{1}(\Omega)} & \left|\sum_{p=1}^{n(\varepsilon)} \varepsilon^{-N}\left(\int_{\Gamma_{p}^{\varepsilon}} w_{\varepsilon} n d s\right) \cdot\left(\int_{Y_{p}^{\varepsilon}} s_{\varepsilon} d x\right)\right| \\
& \leqq C \varepsilon\|\phi\|_{C^{1}(\Omega)}\left\|w_{\varepsilon}\right\|_{H_{\delta}^{1}(\Omega)}\left\|s_{\varepsilon}\right\|_{L^{2}(\Omega)} \leqq C \varepsilon\|\phi\|_{C^{1}(\Omega)} .
\end{aligned}
$$


This proves that $\nabla w_{\varepsilon}$ converges to 0 strongly in $L^{2}(\Omega)^{N}$ (as usual, we identify functions defined in $\Omega_{\varepsilon}$ and their extensions to $\Omega$ defined by Lemma 2.2.2). Now, recalling the definition of $\tilde{S}_{\varepsilon} t_{\varepsilon}$, we obtain

$$
\tilde{S}_{\varepsilon} \boldsymbol{t}_{\varepsilon}=a_{\varepsilon} \sum_{p=1}^{n(\varepsilon)} \varepsilon^{-N}\left(\int_{\Gamma_{p}^{\varepsilon}} \phi u_{\varepsilon} \boldsymbol{n}\right) \chi_{Y_{p}^{2}}(x)+\sum_{p=1}^{n(\varepsilon)} \varepsilon^{-N}\left(\int_{\Gamma_{p}^{\varepsilon}} w_{\varepsilon} \boldsymbol{n}\right) \chi_{Y_{p}^{\varepsilon}}(x) .
$$

The last term in the right-hand side of (115) is easily seen to be bounded by $\left\|\nabla w_{\varepsilon}\right\|_{L^{2}(\Omega)^{*}}$, which tends to 0 , while the first term can be rewritten as

$$
a_{\varepsilon} \sum_{p=1}^{n(\varepsilon)}\left(\varepsilon^{-N} \int_{Y_{p}^{s}} \phi d x\right)\left(\varepsilon^{-N} \int_{\Gamma_{p}^{z}} u_{\varepsilon} n\right) \chi_{Y_{p}^{z}}(x)=a_{\varepsilon} E_{\varepsilon} P_{\varepsilon}\left(\phi(x) \tilde{S}_{\varepsilon} s_{\varepsilon}(x)\right)=\lambda_{\varepsilon} t_{\varepsilon}(x),
$$

up to a small remainder term bounded by $\varepsilon\|\phi\|_{C^{1}(\Omega)}$. This completes the proof of Lemma 3.4.7.

In order to be able to compute explicitly the term $\widetilde{S}_{\varepsilon} t_{\varepsilon}$, the second key idea is to replace the domain of integration $\Omega$ by a larger cube with a periodic boundary condition where a Bloch-wave decomposition of $\boldsymbol{t}_{\varepsilon}$ could be performed. Let $K(\varepsilon)$ be the smallest integer such that the cube $\varepsilon K(\varepsilon) Y$ contains the domain $\Omega$. Let $L$ be the size of the smallest cube containing $\Omega$. As $\varepsilon$ goes to $0, \varepsilon K(\varepsilon)$ converges to $L$. The quasi-eigenvector $\boldsymbol{t}_{\varepsilon}$ is extended by 0 in $\varepsilon K(\varepsilon) Y \backslash \Omega$.

Lemma 3.4.8. Let $\boldsymbol{t}_{\varepsilon}$ be the quasi-eigenvector defined by Lemma 3.4.7. Let $v_{\varepsilon}$ be the solution of

$$
\begin{aligned}
-\Delta v_{\varepsilon} & =0 & & \text { in } \Omega_{\varepsilon}, \\
\frac{\partial v_{\varepsilon}}{\partial n} & =\varepsilon^{-N}\left(\int_{Y_{p}^{\varepsilon}} \boldsymbol{t}_{\varepsilon}(x) d x\right) \cdot \boldsymbol{n} & & \text { on } \Gamma_{p}^{\varepsilon}, \text { for } 1 \leqq p \leqq n(\varepsilon), \\
v_{\varepsilon} & =0 & & \text { on } \partial \Omega,
\end{aligned}
$$

and $w_{\varepsilon}$ that of

$$
\begin{array}{ll}
-\Delta w_{\varepsilon}=0 & \text { in } \varepsilon K(\varepsilon) Y, \\
\frac{\partial w_{\varepsilon}}{\partial n}=\varepsilon^{-N}\left(\int_{Y_{p}^{\varepsilon}} t_{\varepsilon}(x) d x\right) \cdot n & \text { on } \Gamma_{p}^{\varepsilon} \text { for } 1 \leqq p \leqq K(\varepsilon)^{N}, \\
x \rightarrow w_{\varepsilon}(x) \text { is }(\varepsilon K(\varepsilon) Y) \text {-periodic. } &
\end{array}
$$

Then, the difference $\left(v_{\varepsilon}-w_{\varepsilon}\right)$ converges to 0 strongly in $H^{1}(\Omega)$. Therefore,

$$
\tilde{S}_{\varepsilon} \boldsymbol{t}_{\varepsilon}=\sum_{p=1}^{n(\varepsilon)} \varepsilon^{-N}\left(\int_{\Gamma_{p}^{\varepsilon}} w_{\varepsilon} n\right) \chi_{Y_{p}^{\varepsilon}}(x)+\boldsymbol{r}_{\varepsilon}
$$

where $\boldsymbol{r}_{\varepsilon}$ is a remainder term which goes to 0 strongly in $L^{2}(\Omega)^{N}$. 
Proof. Let us define the difference $\delta_{\varepsilon}=w_{\varepsilon}-v_{\varepsilon}$. By combining (116) and (117), it is easily seen that $\delta_{\varepsilon}$ realizes the minimum in $H^{1}\left(\Omega_{\varepsilon}\right)$ of

$$
\min _{\substack{\delta \in \mathbb{H}^{1}\left(\Omega_{\varepsilon}\right) \\ \delta=w_{\varepsilon} \text { on } \delta \Omega}} \int_{\Omega_{\varepsilon}}|\nabla \delta|^{2} d x .
$$

Recall that the sequence $t_{\varepsilon}$ has compact support in a fixed compact subset of $\Omega$. Therefore, there exists a smooth function $\psi \in C^{\infty}\left(\mathbb{R}^{N}\right)$ such that $\psi \equiv 1$ in $\mathbb{R}^{N} \backslash \Omega$ and $\psi \equiv 0$ on the compact support of all $t_{\varepsilon}$. Then, $\psi w_{\varepsilon}$ is an admissible test function in the minimization problem (118), and we have

$$
\int_{\Omega_{\varepsilon}}\left|\nabla \delta_{\varepsilon}\right|^{2} d x \leqq \int_{\Omega_{\varepsilon}} \psi^{2}\left|\nabla w_{\varepsilon}\right|^{2} d x+2 \int_{\Omega_{\varepsilon}} \psi w_{\varepsilon} \nabla w_{\varepsilon} \cdot \nabla \psi d x+\int_{\Omega_{\varepsilon}} w_{\varepsilon}^{2}|\nabla \psi|^{2} d x .
$$

The two last terms in the right-hand side of (119) tend to 0 since, by Lemma 3.4.6, $w_{\varepsilon}$ converges to 0 weakly in $H^{1}(\Omega)$, and strongly in $L^{2}(\Omega)$. On the other hand, multiplying equation (117) by $\phi^{2} w_{\varepsilon}$ and integrating by parts yield

$$
\int_{\Omega_{\varepsilon}} \nabla w_{\varepsilon} \cdot \nabla\left(\psi^{2} w_{\varepsilon}\right) d x=\sum_{p=1}^{n(\varepsilon)} \varepsilon^{-N}\left(\int_{Y_{p}^{\varepsilon}} \boldsymbol{t}_{\varepsilon}(x) d x\right) \cdot\left(\int_{\Gamma_{p}^{\varepsilon}} \psi^{2} w_{\varepsilon} \boldsymbol{n} d s\right)=0,
$$

because the intersection of the supports of $\psi$ and $\boldsymbol{t}_{\varepsilon}$ is empty. Thus, we deduce that

$$
\int_{\Omega_{\varepsilon}} \psi^{2}\left|\nabla w_{\varepsilon}\right|^{2} d x=-2 \int_{\Omega_{\varepsilon}} \psi w_{\varepsilon} \nabla w_{\varepsilon} \cdot \nabla \psi d x
$$

which tends to 0 . This implies that all three terms in the right-hand side of (119) tend to 0 , which proves the desired result.

We are now in a position to compute $\tilde{S}_{\varepsilon} t_{\varepsilon}$ explicitly by decomposing the solution $w_{z}$ of (117) in Bloch waves on the cube $\varepsilon K(\varepsilon) Y$. To do so, we first decompose the quasi-eigenvector $t_{\varepsilon}$, which is piecewise constant in each subcell $Y_{p}^{\varepsilon}$. It is thus amenable to the Bloch-wave decomposition given by Lemma 3.1.2. As already noted in Remark 3.4.1, we can shift the range of the Bloch frequency $j / K(\varepsilon)$ to the interval $\left[-\frac{1}{2} ; \frac{1}{2}\right]^{N}$. This has the advantage of concentrating all the difficulties near 0 . From now on, we replace the summation $\sum_{j=0}^{K(\varepsilon)-1}$ by $\sum_{j=-(K(\varepsilon)-1) / 2}^{(K(\varepsilon)-1) / 2}$ (assuming with no loss of generality that $K(\varepsilon)$ is odd). Finally, by Lemma 3.1.2, we get

$$
\boldsymbol{t}_{\varepsilon}(x)=\sum_{j=-(K(\varepsilon)-1) / 2}^{(K(\varepsilon)-1) / 2} \boldsymbol{t}_{\varepsilon}^{j} e^{2 \pi i \theta_{j}(\varepsilon) \cdot E(x / \varepsilon)} \quad \text { with } \theta_{j}(\varepsilon)=\frac{j}{K(\varepsilon)}
$$

where each $t_{\varepsilon}^{j}$ is a constant vector in $\mathbb{C}^{N}$, and where $E(\cdot)$ denotes the integer part function. On the other hand, the Bloch-wave decomposition in $H^{1}(\varepsilon K(\varepsilon) Y$ ) (as described by Lemma 3.3.4) yields

$$
w_{\varepsilon}(x)=\sum_{j=-(K(\varepsilon)-1) / 2}^{(K(\varepsilon)-1) / 2} w_{\varepsilon}^{j}\left(\frac{x}{\varepsilon}\right)
$$


where each component $w_{\varepsilon}^{j}(y)$ belongs to $H_{*}^{1}\left(e^{2 \pi i \theta_{j}(\varepsilon)}, Y^{*}\right)$. Then, using $(117)$ and the orthogonality of the Bloch waves, and setting $\theta=j / K(\varepsilon)$ and $\boldsymbol{t}_{\varepsilon}^{\theta}=\boldsymbol{t}_{\varepsilon}^{j}$, we deduce that each $w_{\varepsilon}^{j}$ is given by

$$
w_{\varepsilon}^{j}\left(\frac{x}{\varepsilon}\right)=\varepsilon w_{\varepsilon}^{\theta}\left(\frac{x}{\varepsilon}\right)
$$

where $w_{\varepsilon}^{\theta}(y)$ is the unique solution in $H_{\#}^{1}\left(e^{2 \pi i \theta}, Y^{*}\right)$ of

$$
\begin{gathered}
-\Delta w_{\varepsilon}^{\theta}=0 \quad \text { in } Y^{*}, \\
\frac{\partial w_{\varepsilon}^{\theta}}{\partial n}=\boldsymbol{t}_{\varepsilon}^{\theta} \cdot \boldsymbol{n} \quad \text { on } \partial T, \\
y \rightarrow w_{\varepsilon}^{\theta}(y) e^{-2 \pi i \theta \cdot y} \text { is } Y^{*} \text {-periodic. }
\end{gathered}
$$

We can further decompose $w_{\varepsilon}^{\theta}$ in terms of the elementary solutions of the cell problem (64) at the Bloch frequency $\theta$ :

$$
w_{\varepsilon}^{\theta}=\sum_{m=1}^{N}\left(\boldsymbol{t}_{\varepsilon}^{\theta} \cdot \boldsymbol{e}_{m}\right) w_{m}^{\theta}
$$

Note that only the constant vectors $t_{\varepsilon}^{\theta}$ depend on $\varepsilon$, not the functions $w_{m}^{\theta}$. Since $w_{\varepsilon}^{j}$ can be written in terms of simple $\left(e^{2 \pi i \theta}, Y^{*}\right)$-periodic functions, thanks to (123), we have

$$
\sum_{p=1}^{n(\varepsilon)} \varepsilon^{-N}\left(\int_{\Gamma_{p}^{\varepsilon}} w_{\varepsilon}^{j} \boldsymbol{n}\right) \chi_{Y_{p}^{\varepsilon}}(x)=\left(\sum_{m=1}^{N} \boldsymbol{t}_{\varepsilon}^{j} \cdot \boldsymbol{e}_{m} \int_{\partial T} w_{m}^{\theta} \boldsymbol{n}\right) e^{2 \pi i \theta_{j}(\varepsilon) \cdot E(x / \varepsilon)} .
$$

On the other hand, the variational formulations (98) yields the following property of the matrix $A(\theta)$ :

$$
A(\theta) \boldsymbol{e}_{m}=\int_{\partial T} w_{m}^{\theta} n
$$

Thus, by Lemma 3.4.8, we have

$$
\tilde{S}_{\varepsilon} t_{\varepsilon}=\sum_{j=-(K(\varepsilon)-1) / 2}^{(K(\varepsilon)-1) / 2} A\left(\theta_{j}(\varepsilon)\right) t_{\varepsilon}^{j} e^{2 \pi i \theta_{j}(\varepsilon) \cdot E(x / \varepsilon)}+r_{\varepsilon}(x) .
$$

Denoting by $\left(\lambda_{m}(\theta), \boldsymbol{e}_{m}(\theta)\right)_{1 \leqq m \leqq N}$ the eigenvalues and eigenvectors of the matrix $A(\theta)$, we decompose each vector $\boldsymbol{t}_{\varepsilon}^{j}$ as

$$
\boldsymbol{t}_{\varepsilon}^{j}=\sum_{m=1}^{N} t_{\varepsilon}^{j m} \boldsymbol{e}_{m}\left(\theta_{j}(\varepsilon)\right)
$$

and (124) becomes

$$
\tilde{S}_{\varepsilon} \boldsymbol{t}_{\varepsilon}=\sum_{j=-(K(\varepsilon)-1) / 2}^{(\boldsymbol{K}(\varepsilon)-1) / 2} \sum_{m=1}^{N} \lambda_{m}\left(\theta_{j}(\varepsilon)\right) t_{\varepsilon}^{j m} e^{2 \pi i \theta_{j}(\varepsilon) \cdot E(x / \varepsilon)} \boldsymbol{e}_{m}\left(\theta_{j}(\varepsilon)\right)+\boldsymbol{r}_{\varepsilon}(x)
$$


Let us recall that, in view of Lemma $3.4 .7,\left(\tilde{S}_{\varepsilon} t_{\varepsilon}-\lambda_{\varepsilon} t_{\varepsilon}\right)$ converges to 0 strongly in $L^{2}(\Omega)^{N}$. To conclude that, up to a subsequence, $\lambda_{g}$ converges to one of the $\lambda_{m}(\theta)$, the third key idea is to define a modulation of the sequence of quasi-eigenvectors, and to multiply it by the "quasi"-spectral equation satisfied by $\boldsymbol{t}_{\varepsilon}$. The modulation of $\boldsymbol{t}_{\varepsilon}$ is defined by

$$
\mathscr{M}\left(\boldsymbol{t}_{\varepsilon}\right)=\sum_{j=-(K(\varepsilon)-1) / 2}^{(K(\varepsilon)-1) / 2} \sum_{m=1}^{N} \phi_{m}\left(\theta_{j}(\varepsilon)\right) t_{\varepsilon}^{j m} e^{2 \pi i \theta_{j}(\varepsilon) \cdot E(x / \varepsilon)} \boldsymbol{e}_{m}\left(\theta_{j}(\varepsilon)\right)
$$

where the functions $\left(\phi_{m}(\theta)\right)_{1 \leqq m \leqq N}$ are continuous on $\left[-\frac{1}{2} ; \frac{1}{2}\right]^{N}$ and vanish for $\theta=0$. Multiplying $\widetilde{S}_{\varepsilon} t_{\varepsilon}-\lambda_{\varepsilon} t_{\varepsilon}$ by $\mathscr{M}\left(t_{\varepsilon}\right)$ gives

$$
\sum_{j=-(K(\varepsilon)-1) / 2}^{(K(\varepsilon)-1) / 2} \sum_{m=1}^{N}\left(\lambda_{m}\left(\theta_{j}(\varepsilon)\right)-\lambda_{\varepsilon}\right) \phi_{m}\left(\theta_{j}(\varepsilon)\right)\left|t_{\varepsilon}^{j m}\right|^{2}=o(1),
$$

where $o(1)$ is a remainder term which goes to zero. Let us define $N$ positive real measures $\left(\mu_{\varepsilon}^{m}(\theta)\right)_{1 \leqq m \leqq N}$ on $\left[-\frac{1}{2} ; \frac{1}{2}\right]^{N}$ by

$$
\mu_{\varepsilon}^{m}(\theta)=(\varepsilon K(\varepsilon))^{N} \sum_{j=-(K(\varepsilon)-1) / 2}^{(K(\varepsilon)-1) / 2}\left|t_{\varepsilon}^{j m}\right|^{2} \delta_{\theta=\theta_{j}(\varepsilon)}
$$

where each $\delta_{\theta=\theta_{j}(\varepsilon)}$, with $\theta_{j}(\varepsilon)=j / K(\varepsilon)$ is a Dirac mass. We call these measures the Bloch measures associated with the sequence $t_{\varepsilon}$ (they are very similar to the well-known Wigner, or semi-classical, measures; see [16] and [20]). The fundamental equation (125) can be stated as

$$
\sum_{m=1}^{N} \int_{[-1 / 2 ; 1 / 2]^{N}}\left(\lambda_{m}(\theta)-\lambda_{\varepsilon}\right) \bar{\phi}_{m}(\theta) \mu_{\varepsilon}^{m}(d \theta)=o(1) .
$$

Since $\sum_{j}(\varepsilon K(\varepsilon))^{N}\left|t_{\varepsilon}^{j}\right|^{2}=\left\|t_{\varepsilon}\right\|_{L^{2}(\Omega)}^{2}=1$, each $\mu_{\varepsilon}^{m}$ is a bounded sequence of positive finite measures. Up to a subsequence, each converges in the sense of vague measures to a positive limit $\mu^{m}$, and we have

$$
\sum_{m=1}^{N} \int_{[-1 / 2 ; 1 / 2]^{N}} \mu^{m}(d \theta)=\lim _{\varepsilon \rightarrow 0} \sum_{m=1}^{N} \int_{[-1 / 2 ; 1 / 2]^{N}} \mu_{\varepsilon}^{m}(d \theta)=1 .
$$

This implies that at least one of the $\mu^{m}$ is a non-zero finite measure. Since the eigenvalues $\lambda_{m}(\theta)$ are continuous functions of $\theta$, except at 0 where $\phi_{m}(\theta)$ is purposely chosen equal to 0 , we can pass to the limit in (127) to obtain

$$
\int_{[-1 / 2 ; 1 / 2]^{*}}\left(\lambda_{m}(\theta)-\lambda\right) \bar{\phi}_{m}(\theta) \mu^{m}(d \theta)=0
$$

for any $m$. By varying the continuous function $\phi_{m}(\theta)$, we obtain

$$
\lambda_{m}(\theta)=\lambda \quad \mu^{m} \text {-almost everywhere on }\left[-\frac{1}{2} ; \frac{1}{2}\right]^{N} \backslash\{0\} .
$$

Let us assume that, at least one of the limit Bloch measures $\mu^{m}$ is not a Dirac mass concentrated at 0 (this means that the sequence $s_{\varepsilon}$ does actually oscillate on the 
microscopic scale $\varepsilon$ ). Then, (128) implies that there exists a point $\theta_{0} \neq 0$ such that $\lambda_{m}\left(\theta_{0}\right)=\lambda$, which proves that $\lambda$ belongs to the Bloch-wave spectrum $\bigcup_{m=1}^{N}\left[a_{m}, b_{m}\right]$.

Step 4: Microscopic oscillations of the eigenvectors on a length scale larger than 8 . The last case to be considered after the first three steps is that of a sequence of eigenvectors $s_{\varepsilon}$ satisfying the same assumptions as in the third step (i.e., converging weakly to zero in $L^{2}(\Omega)^{N}$ and such that there exist a constant $c>0$ and a subset $\bar{\omega} \subset \Omega$ with $\left.\lim _{\varepsilon \rightarrow 0}\left\|s_{\varepsilon}\right\|_{L^{2}(\omega)} \geqq c>0\right)$, and furthermore having the property that the sequence of quasi-eigenvectors $t_{\varepsilon}$ has all its Bloch measures $\mu^{m}$ equal to multiples of the Dirac mass at zero. This means that, in $\omega$, the sequence $s_{\varepsilon}$ oscillates on a scale much larger than $\varepsilon$, yet still smaller than 1 . In this case, we do not care about the precise length scale, but we are only interested in the directions of oscillations, represented by the variable $\xi \in S^{N}$ (a fact reminiscent of the $H$-measures of P. GÉrARD [15] and L. TARTAR [37]). In the present situation some Bloch frequencies are negligible, as stated in the next lemma.

Lemma 3.4.9. Since $t_{\varepsilon}$ converges weakly to 0 in $L^{2}(\Omega)^{N}$, and since the Bloch measures $\left(\mu^{m}\right)_{1 \leqq m \leqq N}$, which are limits of the sequences $\left(\mu_{\varepsilon}^{m}\right)_{1 \leqq m \leqq N}$ defined from $\boldsymbol{t}_{\varepsilon}$ by (126), are reduced to multiples of the Dirac mass at zero, it follows that

$$
\lim _{\varepsilon \rightarrow 0}\left|t_{\varepsilon}^{j(\varepsilon)}\right|=0
$$

for any sequence of multi-integers $j(\varepsilon)$ such that $\lim _{\varepsilon \rightarrow 0} j(\varepsilon) / K(\varepsilon)=\theta \neq 0$. Furthermore, there exists a sequence of integers $J(\varepsilon)$ such that

$$
\begin{gathered}
\lim _{\varepsilon \rightarrow 0} J(\varepsilon)=+\infty, \quad \lim _{\varepsilon \rightarrow 0} \frac{J(\varepsilon)}{K(\varepsilon)}=0, \\
\lim _{\varepsilon \rightarrow 0} \sum_{J(\varepsilon) \leqq|j| \leqq K(\varepsilon)}\left|t_{\varepsilon}^{j}\right|^{2}=0 .
\end{gathered}
$$

Proof. Let $j(\varepsilon)$ be a sequence of multi-indexes such that $j(\varepsilon) / K(\varepsilon)$ converges to a non-zero limit $\theta$ and that $\left|\boldsymbol{t}_{E}^{j(\varepsilon)}\right|$ converges to a strictly positive limit. Then, it is easily seen that one of the Bloch measures $\mu^{m}$ must be supported at the point $\theta$, which contradicts the hypothesis that it is a Dirac mass at the origin. Eventually, (129) is deduced from a standard diagonalization argument.

Lemma 3.4.9 shows that $\boldsymbol{t}_{\varepsilon}$ can be rewritten as

$$
\boldsymbol{t}_{\varepsilon}(x)=\sum_{0<\langle j| \leqq J(\varepsilon)} \boldsymbol{t}_{\varepsilon}^{j} e^{2 \pi i \theta_{j}(\varepsilon) \cdot E(x / \varepsilon)}+\boldsymbol{r}_{\varepsilon}^{\prime}(x),
$$

where $\boldsymbol{r}_{\varepsilon}^{\prime}$ is another remainder term which goes to 0 strongly in $L^{2}(\Omega)^{N}$ (the term of order $j=0$ is also incorporated into the remainder since $t_{\varepsilon}$ converges weakly to 0 in 
$\left.L^{2}(\Omega)^{N}\right)$. We can proceed as in the third step; multiplying ${\widetilde{S_{\varepsilon}}}_{t_{\varepsilon}}-\lambda_{\varepsilon} t_{\varepsilon}$ by a modulation of $\boldsymbol{t}_{\varepsilon}-\boldsymbol{r}_{\varepsilon}^{\prime}$, we obtain

$$
\sum_{0<|j| \leqq J(\varepsilon)} \sum_{m=1}^{N}\left(\lambda_{m}\left(\theta_{j}(\varepsilon)\right)-\lambda_{\varepsilon}\right) \bar{\phi}_{m}\left(\theta_{j}(\varepsilon)\right)\left|\boldsymbol{t}_{\varepsilon}^{j m}\right|^{2}=o(1),
$$

where each function $\phi_{m}(\theta)$ now depends only on the direction of the vector $\theta$, namely,

$$
\phi_{m}(\theta)=\hat{\phi}_{m}(\theta /|\theta|),
$$

where $\hat{\phi}(\xi)$ is a continuous function of $\xi$ on the unit sphere $S^{N}$. The essential ingredient for the sequel is the continuity of the matrix $A(\theta)$ near zero along the rays $\xi=\theta /|\theta|$, provided by Proposition 3.4.4. Since $J(\varepsilon) / K(\varepsilon)$ goes to zero with $\varepsilon$, we have

$$
\lambda_{m}\left(\theta_{j}(\varepsilon)\right)=\hat{\lambda}_{m}\left(\frac{j}{|j|}\right)+o(1)
$$

uniformly for $0<|j| \leqq J(\varepsilon)$, where $\hat{\lambda}_{m}(\xi)$ are the eigenvalues of the continuous matrix $\hat{A}(\xi)$. In other words, the fundamental equation (130) can be rewritten as

$$
\sum_{0<|j| \leqq J(\varepsilon)} \sum_{m=1}^{N}\left(\hat{\lambda}_{m}\left(\frac{j}{|j|}\right)-\lambda_{\varepsilon}\right) \overline{\hat{\phi}_{m}}\left(\frac{j}{|j|}\right)\left|t_{\varepsilon}^{j m}\right|^{2}=o(1) .
$$

Let us define another family of positive real measures $\left(v_{\varepsilon}^{m}(\xi)\right)_{1 \leqq m \leqq N}$ on $S^{N}$ by

$$
v_{\varepsilon}^{m}(\xi)=(\varepsilon K(\varepsilon))^{N} \sum_{0<|j| \leqq J(\varepsilon)}\left|t_{\varepsilon}^{j m}\right|^{2} \delta_{\xi=j|| j \mid},
$$

where each $\delta_{\xi=j /|j|}$ is a Dirac mass. We call these measures rescaled Bloch measures. Since $\sum_{j}\left(\varepsilon(K(\varepsilon))^{N}\left|\boldsymbol{t}_{\varepsilon}^{j}\right|^{2}=1\right.$, each $v_{\varepsilon}^{m}$ is also a bounded sequence of positive finite measures on $S^{N}$. Up to a subsequence, they converge in the sense of vague measures to limits $v^{m}$ which satisfy

$$
\int_{S^{N}} \sum_{m=1}^{N} v^{m}(d \xi)=1
$$

Since the eigenvalues $\left(\hat{\lambda}_{m}(\xi)\right)_{1 \leqq m \leqslant N}$ of $\widehat{A}(\xi)$ are continuous by virtue of Proposition 3.4.4, we can pass to the limit to obtain

$$
\int_{S^{N}}\left(\hat{\lambda}_{m}(\xi)-\lambda\right) \overline{\hat{\phi}_{m}(\xi)} v^{m}(d \xi)=0
$$

for any $m$. This proves that $\hat{\lambda}_{m}(\xi)=\lambda, v^{m}$-almost everywhere. Since all the rescaled Bloch measures $v^{m}$ cannot be zero, there exists a direction $\xi_{0}$ such that $\hat{\lambda}_{m}\left(\xi_{0}\right)=\lambda$, which proves that $\lambda$ belongs to the Bloch spectrum $\bigcup_{m=1}^{N}\left[a_{m}, b_{m}\right]$. 
Proof of Corollary 3.2.11. If $\Omega$ is a parallelepiped, $] 0, L_{1}[\times] 0, L_{2}[\times \cdots] 0, L_{N}[$, where the $\left(L_{m}\right)_{1 \leqq m \leqq N}$ are positive integers, and if the sequence of periods is $\varepsilon_{n}=1 / n$, then it is easily seen in the previous proof that, for a periodic boundary condition, the case of a sequence of eigenvectors concentrating on the boundary cannot occur since the domain $\Omega$ is actually a torus with a finite number of entire cells. Furthermore, for the same parallelepiped with a Dirichlet boundary condition, this statement still holds at the price of assuming that the unit tube in the periodic cell has cubic symmetry. Indeed, by skew symmetry with respect to the boundary, one can extend the solution $u_{\varepsilon}$ of (109) to a periodic solution of the same equation in a new domain of size twice that of $\Omega$. A simpler proof is even available when $\Omega$ is the unit torus $[0,1]^{N}$ with $\varepsilon_{n}=1 / n$. In this case, $\Omega$ is precisely equal to $\left[0, \varepsilon_{n} K\left(\varepsilon_{n}\right)\right]^{N}$ with $K\left(\varepsilon_{n}\right)=n$. Thus, the operator $S_{\varepsilon_{n}}$ obviously coincides, up to an isomorphism, with the homogenized operator $S^{K\left(\varepsilon_{n}\right)}$. Therefore, the limit spectrum $\sigma_{\infty}$ reduces to $\lim _{K \rightarrow+\infty} \sigma\left(S^{K}\right)$ as expected.

Acknowledgments. Part of this work was carried out while G. ALLAIRE was visiting the Department of Mathematical Engineering of the University of Chile with the support of the French Scientific Commitee ECOS and the Commission for the European Communities through Project CI1*-CT92-0046. C. ConCA is partially supported by Fondecyt under grant No. 19404-94 and by D.T.I. through grant E3099-9425.

\section{References}

1. F. Aguirre \& C. CONCA, Eigenfrequencies of a tube bundle immersed in a fluid, Appl. Math. Optim. 18, 1-38 (1988).

2. G. AllaIRE, Homogenization and two-scale convergence, SIAM J. Math. Anal. 23, 1482-1518 (1992).

3. G. AlLaire \& C. CONCA, Boundary layers in the homogenization of a spectral problem in fluid-solid structures, Submitted to SIAM J. Math. Anal.

4. G. Allaire \& C. CONCA, Analyse asymptotique spectrale de léquation des ondes. Homogénéisation par ondes de Bloch. Complétude du spectre de Bloch. C. R. Acad. Sci. Paris I, 321, 293-298, 557-562 (1995).

5. N. BaKhVAlov \& G. PANASENKo, Homogenization: averaging processes in periodic media, Kluwer, Dordrecht (1990).

6. A. Bensoussan, J. L. Lions \& G. Papanicolaou, Asymptotic analysis for periodic structures, North-Holland, Amsterdam (1978).

7. F. BLOCH, Über die Quantenmechanik der Electronen in Kristallgittern, Z. Phys. 52, $555-600(1928)$.

8. A. BRAIDES, Homogenization of some almost periodic coercive functionals, Rend. Accad. Naz. Sci. XL, 103, 313-322 (1985).

9. D. Cioranescu \& J. Saint Jean Paulin, Homogenization in open sets with holes, J. Math. Anal. Appl. 71, 590-607 (1979).

10. C. Conca, J. Planchard \& M. VANninathan, Limiting behaviour of a spectral problem in fluid-solid structures, Asymptotic Analysis 6, 365-389 (1993).

11. C. Conca, J. Planchard \& M. Vanninathan, Fluids and periodic structures, Wiley \& Masson, Paris (1995).

12. C. CONCA \& M. VANNINATHAN, A spectral problem arising in fluid-solid structures, Comput. Methods Appl. Mech. Engrg. 69, 215-242 (1988). 
13. E. DE GIORGI, G-operators and $\Gamma$-convergence, Proceedings of the International Congress of Mathematicians (Warsaw, August 1983), PWN Polish Scientific Publishers and North Holland, 1175-1191 (1984).

14. I. M. GELFAND, Expansion in series of eigenfunctions of an equation with periodic coefficients, Dokl. Akad. Nauk. SSSR 73, 1117-1120 (1950).

15. P. Gérard, Microlocal defect measures, Comm. Partial Diff. Eqs 16, 1761-1794 (1991).

16. P. GÉRARD, Mesures semi-classiques et ondes de Bloch, in Séminaire Equations aux Dérivées Partielles 1990-1991, 16, Ecole Polytechnique, Palaiseau (1991).

17. G. Geymonat, S. MUller \& N. Triantafyllidis, Homogenization of nonlinearly elastic materials, microscopic bifurcation and macroscopic loss of rank-one convexity, Arch. Rational Mech. Anal. 122, 231-290 (1993).

18. T. KATO, Perturbation theory for linear operators, Springer-Verlag, Berlin (1966).

19. S. KeSAVAN, Homogenization of elliptic eigenvalue problems, I and II, Appl. Math. Optim. 5, 153-167, 197-216 (1979).

20. P. L. Lions \& T. Paul, Sur les mesures de Wigner, to appear in Revista Math. Iberoamer. (1994).

21. S. MÜLLER, Homogenization of nonconvex integral functionals and cellular elastic materials, Arch. Rational Mech. Anal. 99, 189-212 (1987).

22. F. Murat, $H$-convergence, Séminaire d'Analyse Fonctionnelle et Numérique de l'Université d'Alger, mimeographed notes (1978). English translation in "Topics in the mathematical modeling of composite materials", R. V. KoHN, ed., Birkhäuser, Boston.

23. G. NGUETSENG, A general convergence result for a functional related to the theory of homogenization, SIAM J. Math. Anal., 20, 608-623 (1989).

24. F. ODEH \& J. KELLER, Partial differential equations with periodic coefficients and Bloch waves in crystals, J. Math. Phys. 5, $1499-1504$ (1964).

25. O. A. OleiniK, A. S. Shamaev \& G. A. Yosifian, On the limiting behaviour of a sequence of operators defined in different Hilbert spaces, Uspekhi Math. Nauk. 44, 157-158 (1989).

26. O. A. Oleinik, A. S. Shamaev \& G. A. Yosifian, Mathematical problems in elasticity and homogenization, North-Holland, Amsterdam (1992).

27. J. Planchard, Eigenfrequencies of a tube-bundle placed in a confined fluid, Comput. Methods Appl. Mech. Engrg. 30, 75-93 (1982).

28. J. Planchard, Global behaviour of large elastic tube-bundles immersed in a fluid, Comput. Mech. 2, 105-118 (1987).

29. M. REED \& B. Simon, Methods of modern mathematical physics, IV, Analysis of operators, Academic Press, New York (1978).

30. F. RELLICH, Störungstheorie der Spektralzerlegung, Math. Ann. 113, 600-619 (1937).

31. J. SÁNCHEZ-HUBERT \& E. SÁNCHEZ-PALENCIA, Vibration and coupling of continuous systems. Asymptotic methods, Springer-Verlag, Berlin (1989).

32. J. SÁNCHEZ-HubERT \& N. TURBE, Ondes élastiques dans une bande périodique, RAIRO Modèl. Math. Anal. Num. 20, 539-561 (1986).

33. E. SÁNCHEZ-PALENCIA, Non homogeneous media and vibration theory, Lecture Notes in Physics 127, Springer-Verlag (1980).

34. F. SANTOSA \& M. VOGELIUS, First-order corrections to the homogenized eigenvalues of a periodic composite medium, SIAM J. Appl. Math. 53, 1636-1668 (1993).

35. S. SPAGNOLO, Convergence in energy for elliptic operators, Numerical solutions of partial differential equations III Synspade 1975, B. HUBBARD, ed., Academic Press, New York (1976).

36. L. TARTAR, Cours Peccot au College de France, unpublished, partially written in [22] (March 1977). 
37. L. TARTAR, H-measures, a new approach for studying homogenization, oscillations and concentration effects in partial differential equations, Proc. Roy. Soc. Edinburgh 115A, 193-230 (1990).

38. M. VANNINATHAN, Homogenization and eigenvalue problems in perforated domains, Proc. Indian Acad. Sci. Math. Sci. 90, 239-271 (1981).

39. C. WILCOx, Theory of Bloch waves, J. Anal. Math. 33, 146-167 (1978).

\section{Commissariat à l' Energie Atomique} DRN/DMT/SERMA, C.E. Saclay 91191 Gif sur Yvette, France and

Departamento de Ingeniería Matemática Universidad de Chile, Casilla 170/3 Santiago 